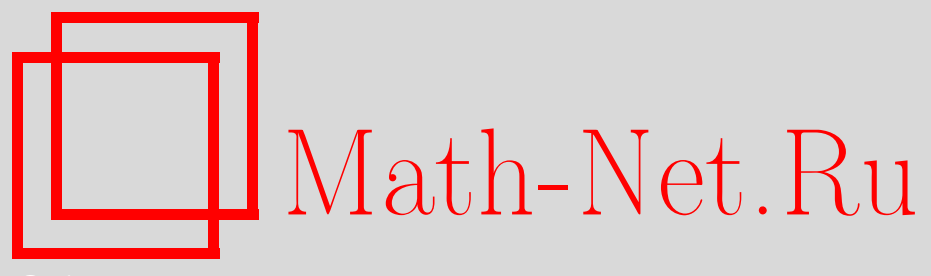

А. Л. Делицын, О постановке краевых задач для системы уравнений Максвелла в цилиндре и их разрешимости, Изв. РАН. Сер. матем., 2007, том 71, выпуск 3, 61112

DOI: https://doi.org/10.4213/im566

Использование Общероссийского математического портала Math-Net.Ru подразумевает, что вы прочитали и согласны с пользовательским соглашением http://www . mathnet.ru/rus/agreement

Параметры загрузки:

IP : 3.85 .7 .115

26 апреля 2023 г., 18:30:27

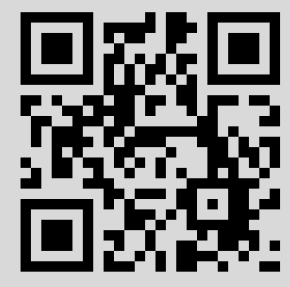




\title{
А. Л. Делицын \\ О постановке краевых задач для системы уравнений Максвелла в цилиндре и их разрешимости
}

\begin{abstract}
Исследованы две задачи теории волноводов: задача об излучении электромагнитных волн в регулярном волноводе с переменным в поперечном сечении заполнением и задача о дифракции электромагнитной волны на рассеивателе в полом волноводе. Рассмотрены постановка условий излучения и разрешимость краевой задачи для системы уравнений Максвелла в цилиндре. Изучены некоторые спектральные задачи, связанные с постановкой условий излучения.

Библиография: 42 наименования.
\end{abstract}

\section{Введение}

Система уравнений Максвелла состоит из восьми уравнений для шести неизвестных функций - координат электрического и магнитного полей. При постановке краевых задач электродинамики (см., например, [1], [2, гл. 6], [3, гл. 7]) принято отмечать, что уравнения

$$
\operatorname{div} \varepsilon E=4 \pi \rho, \quad \operatorname{div} \mu H=0
$$

являются следствиями уравнений

$$
\operatorname{rot} H=\frac{1}{c} \frac{\partial}{\partial t} \varepsilon E+\frac{4 \pi}{c} j, \quad \operatorname{rot} E=-\frac{1}{c} \frac{\partial}{\partial t} \mu H
$$

при условии, что начальные данные удовлетворяют соотношениям (0.1) при $t=t_{0}$, а ток и заряды удовлетворяют уравнению непрерывности

$$
\frac{\partial \rho}{\partial t}+\operatorname{div} j=0
$$

(Решения системы уравнений предполагаются достаточно гладкими для того, чтобы их можно было дифференцировать требуемое количество раз.) В то же время, в известных автору курсах не принято акцентировать внимание на том факте, что любое из уравнений Максвелла является следствием трех других уравнений (при условии, что оно выполняется на гиперплоскости, отвечающей координате, входящей в уравнение как параметр). Например, уравнение

$$
(\operatorname{rot} H)_{z}=\frac{1}{c} \frac{\partial}{\partial t} \varepsilon E_{z}+\frac{4 \pi}{c} j_{z}
$$

Работа выполнена при поддержке РФФИ (грант № 06-01-00146).

(C) А. Л. ДЕлицын, 2007 
является следствием уравнения

$$
\operatorname{div} \varepsilon E=4 \pi \rho
$$

и уравнений

$$
\begin{aligned}
& (\operatorname{rot} H)_{x}=\frac{1}{c} \frac{\partial}{\partial t} \varepsilon E_{x}+\frac{4 \pi}{c} j_{x} \\
& (\operatorname{rot} H)_{y}=\frac{1}{c} \frac{\partial}{\partial t} \varepsilon E_{y}+\frac{4 \pi}{c} j_{y}
\end{aligned}
$$

при условии, что (0.4) выполняется на некоторой гиперплоскости $\left(x=x_{0}\right)$. Справедливость этого утверждения легко проверить непосредственно. Проще это увидеть, если перейти от векторной формы записи двух уравнений Максвелла

$$
\operatorname{div} \varepsilon E=4 \pi \rho, \quad \operatorname{rot} H=\frac{1}{c} \frac{\partial}{\partial t} \varepsilon E+\frac{4 \pi}{c} j
$$

к введенной Г. Минковским тензорной форме [2]

$$
\frac{\partial F^{i k}}{\partial x^{k}}=\frac{4 \pi}{c} j^{i}, \quad i, j=0,1,2,3,
$$

где тензору $F^{i k}$ соответствует матрица

$$
F=\left(\begin{array}{cccc}
0 & \varepsilon E_{x} & \varepsilon E_{y} & \varepsilon E_{z} \\
-\varepsilon E_{x} & 0 & H_{z} & -H_{y} \\
-\varepsilon E_{y} & -H_{z} & 0 & H_{x} \\
-\varepsilon E_{z} & H_{y} & -H_{x} & 0
\end{array}\right)
$$

$x^{0}=c t, x^{1}=x, x^{2}=y, x^{3}=z$. Указанное утверждение является записью хорошо известного уравнения

$$
\frac{\partial}{\partial x^{i}}\left(\frac{\partial F^{i k}}{\partial x^{k}}-\frac{4 \pi}{c} j^{i}\right)=0,
$$

которое является следствием антисимметричности тензора $F^{i k}\left(F^{i k}=-F^{k i}\right)$, системы уравнений (0.9) и уравнения непрерывности

$$
\frac{\partial j^{i}}{\partial x^{i}}=0 .
$$

Таким образом, так же, как уравнения (0.1) для дивергенций полей $\varepsilon E$ и $\mu H$ можно рассматривать в качестве следствий уравнений $(0.2)$, любое из уравнений Максвелла можно считать следствием трех других уравнений. Тем не менее, традиционно, при рассмотрении стационарной системы уравнений Максвелла, принято выделять особую роль уравнений (0.2) (см. [1], [3]), называемых даже в указанных монографиях основными. В случае, когда заряды и поля имеют гармоническую зависимость от времени вида $e^{-i \omega t}$, после сокращения на экспоненциальный множитель $e^{-i \omega t}$ система уравнений Максвелла принимает вид

$$
\begin{gathered}
\operatorname{rot} E=i k \mu H, \quad \operatorname{rot} H=-i k \varepsilon E+\frac{4 \pi}{c} j, \\
\operatorname{div} \mu H=0, \quad \operatorname{div} \varepsilon E=4 \pi \rho .
\end{gathered}
$$


При этом уравнения (0.13) являются непосредственными следствиями уравнений (0.12). Для постановки краевой задачи используют, как правило, систему (0.12). В гл. 1 настоящей работы показано, что для задач, в которых одна из пространственных координат является "выделенной", возможен принципиально иной метод их постановки.

Пусть коэффициенты системы уравнений Максвелла не зависят от координаты $z: \varepsilon=\varepsilon(x, y), \mu=\mu(x, y)$. В этом случае возможен выбор в качестве основных не уравнений (0.12), а тех шести уравнений, в которые входят производные по координате $z$ :

$$
\begin{gathered}
(\operatorname{rot} H)_{x}=-i k(\varepsilon E)_{x}+\frac{4 \pi}{c} j_{x}, \\
(\operatorname{rot} H)_{y}=-i k(\varepsilon E)_{y}+\frac{4 \pi}{c} j_{y}, \\
\operatorname{div} \varepsilon E=4 \pi \rho
\end{gathered}
$$

составляющих первую подсистему, и

$$
\begin{gathered}
(\operatorname{rot} E)_{x}=i k(\mu H)_{x}, \\
(\operatorname{rot} E)_{y}=i k(\mu H)_{y}, \\
\operatorname{div} \mu H=0
\end{gathered}
$$

составляющих вторую.

Два оставшихся уравнения

$$
(\operatorname{rot} H)_{z}=-i k \varepsilon E_{z}+\frac{4 \pi}{c} j_{z}, \quad(\operatorname{rot} E)_{z}=i k \mu H_{z}
$$

являются следствиями соответственно уравнений $(0.14)-(0.16)$ и $(0.17)-(0.19)$ при условии, что они выполняются при некотором значении координаты $z$.

Задачу естественно рассматривать не относительно векторов $E$ и $H$, а относительно векторов $A_{1}=\left\{\mu H_{x}, \mu H_{y}, E_{z}\right\}$ и $A_{2}=\left\{\varepsilon E_{x}, \varepsilon E_{y}, H_{z}\right\}$.

В гл. 1 мы продемонстрируем, к каким существенным упрощениям приводит данный метод при исследовании определенных электродинамических задач. Задачи, в которых одна из координат имеет “выделенный” характер, возникают в теории регулярных волноводов [4]. Под задачами теории регулярных волноводов будем понимать краевые задачи для стационарной системы уравнений Максвелла в цилиндре $Q=\{(x, y) \in \Omega, z \in(-\infty,+\infty)\}$, где $\Omega$ - ограниченная область, с коэффициентами $\varepsilon$ и $\mu$, не зависящими от координаты $z$. В качестве граничных условий используем условия, соответствующие идеально проводящей стенке [1], [2]. Одна из основных задач теории регулярных волноводов [4] заключается в исследовании свойств решений однородной системы уравнений Максвелла специального вида

$$
H=H(x, y) e^{i \gamma z}, \quad E=E(x, y) e^{i \gamma z},
$$

к поиску которых приводит применение метода Фурье. Поиск решений подобного вида приводит при традиционной постановке задачи относительно вихревой подсистемы уравнений Максвелла к необходимости рассматривать спектральные задачи для системы уравнений

$$
\operatorname{rot} E=i k \mu H, \quad \operatorname{rot} H=-i k \varepsilon E .
$$


Спектральная задача, возникающая при подстановке в уравнения (0.21) векторов $E=E(x, y) e^{i \gamma z}, H=H(x, y) e^{i \gamma z}$, имеет вид

$$
\mathcal{A} u=\gamma \mathcal{B} u
$$

где $u=(E, H), \mathcal{A}$ - дифференциальный оператор с бесконечномерным ядром, $\mathcal{B}$ - вырожденная матрица. Спектральная задача относительно $u, \gamma$ рассматривается в поперечном сечении $\Omega$. Автору не известны какие-либо результаты, относящиеся к подобной спектральной задаче, за исключением частного случая

$$
\varepsilon=\varepsilon_{0} I, \quad \mu=\mu_{0} I,
$$

где $\varepsilon_{0}, \mu_{0}$ - положительные константы, $I$ - единичная матрица. В этом случае хорошо известно (см., например, [1]), что собственные значения $\gamma$ выражаются через собственные значения двумерного оператора Лапласа

$$
\Delta_{\perp}=\frac{\partial^{2}}{\partial x^{2}}+\frac{\partial^{2}}{\partial y^{2}}
$$

с условиями Дирихле или Неймана. Более точно, $\gamma_{n}= \pm \sqrt{\lambda_{i n}-k^{2}}, \quad i=1,2$, где $\lambda_{1 n}-$ собственное значение задачи Дирихле

$$
-\Delta_{\perp} \chi=\lambda_{1} \chi, \quad(x, y) \in \Omega,\left.\quad \chi\right|_{\partial \Omega}=0
$$

a $\lambda_{2 n}-$ собственное значение задачи Неймана

$$
-\Delta_{\perp} \psi=\lambda_{2} \psi, \quad(x, y) \in \Omega,\left.\quad \frac{\partial \psi}{\partial \boldsymbol{n}}\right|_{\partial \Omega}=0
$$

Собственные векторы задачи для уравнений (0.21) выражаются через функции $\psi_{n}, \chi_{n}$. Заметим, что подобный простой вид собственных значений и собственных векторов задачи имеет место только в случае коэффициентов вида $\varepsilon=\varepsilon_{0} I, \mu=\mu_{0} I$ (с физической точки зрения этот случай соответствует полому волноводу, не заполненному средой). Ситуация принципиально меняется даже в случае постоянных матриц $\varepsilon$ и $\mu$ вида

$$
\varepsilon=\varepsilon_{i} \delta_{i j}, \quad \mu=I,
$$

где $\delta_{i j}$ - символ Кронекера, причем $\varepsilon_{1}>0, \varepsilon_{2}>0, \varepsilon_{3}>0$. В 55 мы приводим пример задачи, допускающей аналитическое решение, с постоянными коэффициентами указанного типа, в которой возникают комплексные собственные значения. Задача, очевидно, усложняется еще больше в случае переменных коэффициентов $\varepsilon, \mu$.

В случае переменных коэффициентов в известных автору работах не рассматривается спектральная задача для системы уравнений первого порядка, а рассматриваются две различные системы уравнений второго порядка, следующие из (0.21) (точнее, из (0.22)), для которых и ставятся краевые условия. В работах [5]-[7] спектральная задача ставится для системы уравнений второго порядка относительно продольных координат $E_{z}$ и $H_{z}$. Основная сложность исследования подобной задачи заключается в нелинейном вхождении 
спектрального параметра $\gamma$. Приведем возникающую систему уравнений относительно $E_{z}, H_{z}$ в частном случае $\varepsilon=\varepsilon(x, y) I, \mu=\mu(x, y) I$ :

$$
\begin{aligned}
\frac{\partial}{\partial x} & \frac{k \varepsilon}{k^{2} \varepsilon \mu-\gamma^{2}} \frac{\partial E_{z}}{\partial x}+\frac{\partial}{\partial y} \frac{k \varepsilon}{k^{2} \varepsilon \mu-\gamma^{2}} \frac{\partial E_{z}}{\partial y} \\
& +\frac{\partial}{\partial x} \frac{\gamma}{k^{2} \varepsilon \mu-\gamma^{2}} \frac{\partial H_{z}}{\partial y}-\frac{\partial}{\partial y} \frac{\gamma}{k^{2} \varepsilon \mu-\gamma^{2}} \frac{\partial H_{z}}{\partial x}+k \varepsilon E_{z}=0 \\
\frac{\partial}{\partial x} & \frac{k \mu}{k^{2} \varepsilon \mu-\gamma^{2}} \frac{\partial H_{z}}{\partial x}+\frac{\partial}{\partial y} \frac{k \mu}{k^{2} \varepsilon \mu-\gamma^{2}} \frac{\partial H_{z}}{\partial y} \\
& -\frac{\partial}{\partial x} \frac{\gamma}{k^{2} \varepsilon \mu-\gamma^{2}} \frac{\partial E_{z}}{\partial y}+\frac{\partial}{\partial y} \frac{\gamma}{k^{2} \varepsilon \mu-\gamma^{2}} \frac{\partial E_{z}}{\partial x}+k \mu H_{z}=0
\end{aligned}
$$

Эту систему уравнений в работах [5]-[7] дополняют граничными условиями

$$
\left.E_{z}\right|_{\partial \Omega}=0,\left.\quad \frac{\partial H_{z}}{\partial \boldsymbol{n}}\right|_{\partial \Omega}=0 .
$$

Только в случае $\varepsilon=\varepsilon_{0} I, \mu=\mu_{0} I$ задача (0.27)-(0.29) эквивалентна двум спектральным задачам:

$$
\Delta_{\perp} E_{z}+k^{2} \varepsilon_{0} E_{z}=\gamma^{2} E_{z},\left.\quad E_{z}\right|_{\partial \Omega}=0
$$

и

$$
\Delta_{\perp} H_{z}+k^{2} \mu_{0} H_{z}=\gamma^{2} H_{z},\left.\quad \frac{\partial H_{z}}{\partial \boldsymbol{n}}\right|_{\partial \Omega}=0 .
$$

В работах [5]-[7] рассматривалась спектральная задача (0.27)-(0.29) в случае коэффициентов специального вида: $\mu=I$, а $\varepsilon$ - кусочно постоянная функция, принимающая всего два значения:

$$
\varepsilon=\left\{\begin{array}{lll}
\varepsilon_{1} I>0, & \text { если } & (x, y) \in \Omega_{1}, \\
\varepsilon_{2} I>0, & \text { если } & (x, y) \in \Omega_{2},
\end{array} \quad \varepsilon_{1} \neq \varepsilon_{2} .\right.
$$

При этом накладывались ограничения на величину $\varepsilon_{2}-\varepsilon_{1}$. (Подобные задачи принято называть задачами о двухслойных волноводах.) В работах [5]-[7] доказана полнота системы корневых векторов $\left(E_{z}, H_{z}\right)$ в пространстве $\dot{H}^{1}(\Omega) \times$ $H^{1}(\Omega)$ для указанного частного вида функции $\varepsilon$.

Второй метод постановки спектральной задачи заключается в рассмотрении спектральной задачи для системы уравнений второго порядка относительно поперечных координат векторов $E$ и $H$, т. е. вектора $\left(E_{\perp}, H_{\perp}\right)$. Этот метод применялся в работе [8], где в случае, когда сечение цилиндра является кругом, $\varepsilon=\varepsilon(r) I, \mu \equiv I$, доказана полнота системы корневых векторов вида $\left(E_{r}, E_{\phi}, H_{r}, H_{\phi}\right)$. В настоящей работе мы не будем приводить систему уравнений (в силу её громоздкости), для которых в [8] была поставлена спектральная задача. Отметим только, что в силу зависимости коэффициентов системы уравнений только от одной координаты $r$ спектральная задача ставится для системы обыкновенных дифференциальных уравнений. Сложность исследования рассматриваемой задачи заключается в том, что она представляет собой спектральную задачу вида

$$
\mathcal{A} u=\gamma \mathcal{B} u
$$

где $u=\left(E_{\perp}, H_{\perp}\right)$, для пары симметрических, но знаконеопределенных операторов $\mathcal{A}, \mathcal{B}$. 
Наконец, третья возможность заключается в постановке задачи относительно вектора $H$ либо $E$. При этом возникает задача для квадратичного относительно спектрального параметра $\gamma$ операторного пучка вида

$$
\mathcal{A} H+\gamma \mathcal{B} H+\gamma^{2} \mathcal{C} H+k^{2} \mathcal{D} H=0 .
$$

Однако операторы $\mathcal{A}, \mathcal{B}, \mathcal{C}$ являются вырожденными. Поэтому к данной задаче не применимы известные результаты теории квадратичных операторных пучков [9], [10], что отмечалось в монографии [4].

Таким образом, в сколько-нибудь полной постановке вопрос о полноте системы корневых векторов, а также об определении области локализации и асимптотике собственных значений решен не был. В 22 устанавливается, что эти вопросы могут быть решены стандартным образом, если ставить соответствующую спектральную задачу для “правильно" выбранной системы уравнений.

Вторая рассматриваемая в гл. 1 задача (связанная с первой) - это постановка условий излучения и доказательство разрешимости краевой задачи для системы уравнений Максвелла в цилиндре. Для решения этой задачи в известных автору работах использовалась вихревая подсистема (0.2) системы уравнений Максвелла. Первые математически строгие результаты были получены в работах [11]-[13], в которых доказана разрешимость соответствующей краевой задачи для случая постоянных коэффициентов специального вида $\varepsilon=\varepsilon_{0} I$, $\mu=\mu_{0} I$. (Отметим еще раз, что случай, когда $\varepsilon, \mu-$ некоторые постоянные эрмитовы положительно определенные матрицы, даже диагональные, значительно сложнее.) В работах [11], [12] вводится вектор Герца, для которого задача сводится к рассмотрению краевой задачи для уравнения Гельмгольца в цилиндре и исследованию гладкости вектора Герца, позволяющего найти решение исходной задачи. Метод, основанный на постановке вспомогательной задачи для вектора Герца, применим только в случае $\varepsilon=\varepsilon_{0} I, \mu=\mu_{0} I$, где $\varepsilon_{0}>0$, $\mu_{0}>0$ - константы. Существуют другие методы решения этой задачи в случае $\varepsilon=\varepsilon_{0} I, \mu=\mu_{0} I$. Они сводятся к различным формальным схемам метода Фурье либо метода Галёркина [1], в которых учитывается специфическое для данной задачи свойство ортогональности различных подсистем векторов, выбираемых из собственных векторов. В случае переменных коэффициентов задача рассматривается в строгой математической постановке в работе [8], где коэффициенты системы уравнений Максвелла имеют специальный вид: $\varepsilon=\varepsilon(r) I$, $\mu=\mu_{0} I$. В работе [8] доказывается существование приближенного решения со сколь угодно малой невязкой. Под этим подразумевается, что если задача формулируется в виде уравнения $L u=f$, то для любого $\varepsilon>0$ найдется $u_{N}$ такое, что $\left\|L u_{N}-f\right\|<\varepsilon$.

Существуют различные варианты постановки условий излучения в цилиндре. В гл. 1 настоящей работы мы следуем методу постановки условий излучения, изложенному в работе [14]. Он заключается в требовании представимости решения вне носителя токов и зарядов в виде суммы конечного числа решений однородной системы уравнений, имеющих физический смысл бегущих волн, и решения, принадлежащего $L_{2}(Q)$. Доказательство существования решения задачи с подобными краевыми условиями осуществляется стандартным способом в $\S 3$. Основная цель в $\S 3$ заключается в том, чтобы показать, что постановка краевой задачи относительно векторов $\left((\mu H)_{x},(\mu H)_{y}, E_{z}\right)$ и $\left((\varepsilon E)_{x},(\varepsilon E)_{y}, H_{z}\right)$, а не векторов $\left(E_{x}, E_{y}, E_{z}\right)$ и $\left(H_{x}, H_{y}, H_{z}\right)$ снимает трудности в постановке условий излучения и доказательстве разрешимости соответствующей краевой задачи. 
В гл. 2 рассматривается задача о дифракции нормальной волны в цилиндрическом волноводе на ограниченном рассеивателе (эту задачу принято называть задачей о дифракиии в нерегулярном волноводе). Коэффициенты системы уравнений Максвелла вне некоторого шара имеют вид $\varepsilon=I, \mu=I$. При этом в области рассеивателя коэффициенты $\varepsilon$ и $\mu$ зависят от трех координат $x, y, z$. В качестве условий излучения в гл. 2 используются условия излучения, введенные А.Г. Свешниковым при постановке краевой задачи для уравнения Гельмгольца в работе [16] (см. также [15]) и для системы уравнений Максвелла в работе [17]. А. Г. Свешников предложил свести задачу дифракции в бесконечном цилиндре к эквивалентной ей задаче в цилиндре конечной высоты, но с нелокальными краевыми условиями на основаниях цилиндра. В работах [17], [18] предполагалось, что

$$
\varepsilon=\varepsilon_{1}(x, y, z)+i \varepsilon_{2} I, \quad \mu=\mu_{1}(x, y, z)+i \mu_{2} I,
$$

где $\varepsilon_{2}>0$ и $\mu_{2}>0$ - константы (которые могут быть выбраны сколь угодно малыми). С физической точки зрения подобный вид коэффициентов означает наличие поглощения в среде. Из полученных в работах [17], [18] энергетических оценок следует разрешимость рассматриваемой задачи дифракции. В то же время принцип предельного поглощения не применим для задачи дифракции в цилиндре, поскольку в случае $\varepsilon_{2}=0, \mu_{2}=0$ при определенных значениях параметра $k$ могут существовать решения однородной системы уравнений Максвелла, удовлетворяющие условиям $E \in L_{2}(Q), H \in L_{2}(Q)$.

Для постановки задачи в работах [17], [18] фактически используется система уравнений относительно поперечных составляющих электромагнитного поля $\left(E_{\perp}, H_{\perp}\right)$.

В настоящей работе задача рассматривается в случае, когда матрицы диэлектрической и магнитной проницаемостей эрмитовы, ограниченные и положительно определенные. В этом случае задача существенно усложняется и автору не известны работы по ее разрешимости.

Для постановки задачи дифракции нормальной волны на ограниченном рассеивателе в гл. 2 настоящей работы используется традиционный выбор вихревой подсистемы уравнений Максвелла. В отличие от работ [17], [18], задача ставится для вектора $H$. Для исследования разрешимости задачи дифракции рассматривается вспомогательная задача в конечном цилиндре с нелокальными краевыми условиями, полностью аналогичными введенным впервые в работе [16]. Таким образом вопрос о разрешимости задачи дифракции в бесконечном цилиндре сводится к рассмотрению внутренней краевой задачи. При доказательстве разрешимости этой задачи основную роль играет теорема о компактности вложения некоторого векторного функционального пространства в $L_{2}(V)$. В работах [19]-[21] доказана компактность вложения пространств $H_{0}(\operatorname{rot}, V) \cap H(\operatorname{div}, V)$ и $H(\operatorname{rot}, V) \cap H_{0}(\operatorname{div}, V)$ в $L_{2}(V)$ для определенного класса областей $V$. В $\S 9$ настоящей работы вводится подпространство $\mathcal{V}$ пространства $H($ rot $) \cap H($ div $)$, выделяемое системой условий на следы элементов данного пространства, и доказывается компактность вложения $\mathcal{V}$ в $L_{2}(V)$. Введение пространства $\mathcal{V}$ позволяет доказать разрешимость соответствующей внутренней краевой задачи, из которой следует разрешимость задачи дифракции нормальной волны на ограниченном препятствии.

В то же время возможна потеря единственности решения этой задачи. Хорошо известно, что оператор Лапласа в локально деформированном цилиндре с нулевыми условиями Дирихле при определенном виде деформации имеет 
непустой дискретный спектр. Этот факт был доказан в работе [22]. Существует много работ, посвященных описанию дискретного спектра оператора Лапласа в локально деформированном цилиндре. Однако автору не известны работы, в которых рассматривалась аналогичная спектральная задача для системы уравнений Максвелла. В $\S 10$ доказывается непустота дискретного спектра для системы уравнений Максвелла с переменными коэффициентами определенного вида.

\section{Глава 1. Условия излучения и задача возбуждения регулярного волновода}

\section{§ 1. Постановка задачи и выбор уравнений}

Задачей возбуждения регулярного волновода будем называть краевую задачу в цилиндре для неоднородной системы уравнений Максвелла с переменными коэффициентами и некоторыми условиями излучения. Постановка условий излучения является отдельной задачей, которая будет подробно рассматриваться ниже, в 33 .

Рассматривается задача в цилиндре (рис. 1) $Q=\{(x, y) \in \Omega, z \in(-\infty,+\infty)\}$, сечение цилиндра $\Omega$ - ограниченная односвязная область с кусочно гладкой границей. Считаем, что область $\Omega$ принадлежит классу областей, описываемых в $[23$, гл. $1, \S 5,6],[24$, гл. $1, \S 1]$, и для нее справедлива теорема о компактности вложения $H^{1}(\Omega)$ в $L_{2}(\Omega)$.

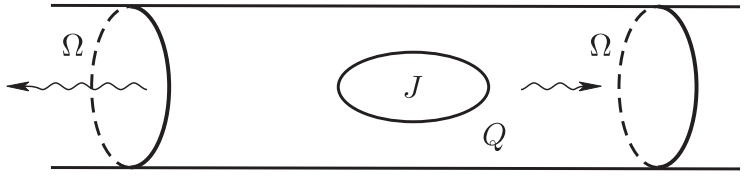

Рис. 1

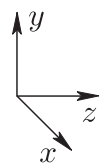

Считаем, что токи, и заряды, и возбуждаемое ими поле имеют гармоническую зависимость $e^{-i \omega t}$ от времени. После сокращения на временной множитель приходим к стационарной системе уравнений Максвелла

$$
\begin{gathered}
\operatorname{rot} E=i k B, \quad \operatorname{rot} H=-i k D+\frac{4 \pi}{c} j, \\
\operatorname{div} B=0, \quad \operatorname{div} D=4 \pi \rho,
\end{gathered}
$$

где $k=\frac{\omega}{c}, D=\varepsilon E, B=\mu H$. Матрицы диэлектрической и магнитной проницаемостей $\varepsilon, \mu$ не зависят от координаты $z: \varepsilon=\varepsilon(x, y), \mu=\mu(x, y)$. Считаем, что матрицы $\varepsilon, \mu$ эрмитовы, положительно определены, кусочно непрерывны и имеют вид

$$
\varepsilon=\left(\begin{array}{ccc}
\varepsilon_{11} & \varepsilon_{12} & 0 \\
\varepsilon_{12}^{*} & \varepsilon_{22} & 0 \\
0 & 0 & \varepsilon_{33}
\end{array}\right), \quad \mu=\left(\begin{array}{ccc}
\mu_{11} & \mu_{12} & 0 \\
\mu_{12}^{*} & \mu_{22} & 0 \\
0 & 0 & \mu_{33}
\end{array}\right)
$$


$\left(\varepsilon_{i j}^{*}\right.$ означает комплексное сопряжение к $\left.\varepsilon_{i j}\right)$,

$$
\begin{gathered}
\alpha \sum_{i=1}^{3}\left|\xi_{i}\right|^{2} \leqslant \sum_{i, j=1}^{3} \varepsilon_{i j} \xi_{i} \xi_{j}^{*} \leqslant \beta \sum_{i=1}^{3}\left|\xi_{i}\right|^{2}, \\
\alpha \sum_{i=1}^{3}\left|\xi_{i}\right|^{2} \leqslant \sum_{i, j=1}^{3} \mu_{i j} \xi_{i} \xi_{j}^{*} \leqslant \beta \sum_{i=1}^{3}\left|\xi_{i}\right|^{2} \quad \forall \xi \in \mathbb{C}^{3},
\end{gathered}
$$

где $\alpha>0, \beta>0$.

На границе цилиндра (всюду, за исключением ребер) поставим условия, соответствующие идеально проводящей стенке:

$$
E \times\left.\boldsymbol{n}\right|_{\partial Q}=0,\left.\quad B \boldsymbol{n}\right|_{\partial Q}=0 .
$$

На кусочно гладких поверхностях разрыва $S$ диэлектрической и магнитной проницаемостей поставим условия сопряжения

$$
\begin{array}{ll}
{\left.[D \boldsymbol{n}]\right|_{S}=0,} & {\left.[E \times \boldsymbol{n}]\right|_{S}=0,} \\
{\left.[B \boldsymbol{n}]\right|_{S}=0,} & {\left.[H \times \boldsymbol{n}]\right|_{S}=0 .}
\end{array}
$$

В качестве “условия на ребре" потребуем выполнения условий

$$
E \in L_{2}^{\text {loc }}, \quad H \in L_{2}^{\text {loc }} .
$$

Считаем, что токи и заряды $j=j(x, y, z), \rho=\rho(x, y, z)$, возбуждающие поле, являются заданными функциями, локализованными в конечной области $\operatorname{supp} j \subset \mathcal{D}, \mathcal{D}=\left\{(x, y) \in \Omega, z \in\left[z_{1}, z_{2}\right]\right\}$, удовлетворяют условиям $j \in H^{1}(Q)$, $\rho \in L_{2}(Q)$ и уравнению непрерывности, которое имеет после сокращения на временной множитель вид

$$
\operatorname{div} j-i \omega \rho=0 .
$$

Для постановки краевой задачи необходимо дополнить (1.1)-(1.4) условиями излучения при $z \rightarrow+\infty, z \rightarrow-\infty$.

Для постановки условий излучения далее мы рассмотрим однородную систему уравнений Максвелла вне области локализации токов.

ЗАмЕчАНиЕ 1.1. Будем называть параметр $k=\frac{\omega}{c}$ также частотой электромагнитного поля.

Введем дополнительные ограничения на параметр $k$. Следуя [1], [4], сформулируем следующее определение.

ОПРЕдЕЛЕНИЕ 1.1. Значения параметра $k$, при которых существуют решения однородной системы уравнений Максвелла в цилиндре $Q$, удовлетворяющие условиям $(1.3),(1.4)$ и не зависящие от координаты $z$, называют критическими частотами или частотами отсечки.

УТВЕРЖДЕНИЕ 1.1. Существует бесконечное число частот отсечки $k_{n}$ с единственной точкой сгущения на бесконечности.

Доказательство утверждения 1.1 будет дано в $\S 6$. Будем считать далее, что частота электромагнитного поля $k$ не совпадает с частотами отсечки. 
Перейдем к изложению предлагаемого в настоящей статье метода (см. также [25], [26]). Основная идея этого метода заключается в том, чтобы записать систему уравнений Максвелла в виде

$$
\begin{aligned}
& \left(\begin{array}{ccc}
0 & i k & \frac{\partial}{\partial x} \\
-i k & 0 & \frac{\partial}{\partial y} \\
-\frac{\partial}{\partial x} & -\frac{\partial}{\partial y} & 0
\end{array}\right)\left(\begin{array}{l}
B_{x} \\
B_{y} \\
E_{z}
\end{array}\right)=\left(\begin{array}{ccc}
\left(\varepsilon^{-1}\right)_{11} & \left(\varepsilon^{-1}\right)_{12} & 0 \\
\left(\varepsilon^{-1}\right)_{12}^{*} & \left(\varepsilon^{-1}\right)_{22} & 0 \\
0 & 0 & \mu_{33}
\end{array}\right) \frac{\partial}{\partial z}\left(\begin{array}{l}
D_{x} \\
D_{y} \\
H_{z}
\end{array}\right), \\
& \left(\begin{array}{ccc}
0 & -i k & \frac{\partial}{\partial x} \\
i k & 0 & \frac{\partial}{\partial y} \\
-\frac{\partial}{\partial x} & -\frac{\partial}{\partial y} & 0
\end{array}\right)\left(\begin{array}{l}
D_{x} \\
D_{y} \\
H_{z}
\end{array}\right)=\left(\begin{array}{ccc}
\left(\mu^{-1}\right)_{11} & \left(\mu^{-1}\right)_{12} & 0 \\
\left(\mu^{-1}\right)_{12}^{*} & \left(\mu^{-1}\right)_{22} & 0 \\
0 & 0 & \varepsilon_{33}
\end{array}\right) \frac{\partial}{\partial z}\left(\begin{array}{l}
B_{x} \\
B_{y} \\
E_{z}
\end{array}\right)+\frac{4 \pi}{c}\left(\begin{array}{c}
-j_{y} \\
j_{x} \\
-c \rho
\end{array}\right), \\
& \frac{\partial}{\partial x}\left(\mu^{-1} B\right)_{y}-\frac{\partial}{\partial y}\left(\mu^{-1} B\right)_{x}+i k \varepsilon_{33} E_{z}=\frac{4 \pi}{c} j_{z}, \\
& \frac{\partial}{\partial x}\left(\varepsilon^{-1} D\right)_{y}-\frac{\partial}{\partial y}\left(\varepsilon^{-1} D\right)_{x}+i k \mu_{33} H_{z}=0 .
\end{aligned}
$$

При рассмотрении краевых задач электродинамики основную роль играет обычно вихревая пара системы уравнений Максвелла. Аналогичную роль играют уравнения (1.6), (1.7).

Введем следующие обозначения:

$$
\begin{gathered}
a_{1}=\left(\begin{array}{ccc}
0 & i k & \frac{\partial}{\partial x} \\
-i k & 0 & \frac{\partial}{\partial y} \\
-\frac{\partial}{\partial x} & -\frac{\partial}{\partial y} & 0
\end{array}\right), \quad a_{2}=\left(\begin{array}{ccc}
0 & -i k & \frac{\partial}{\partial x} \\
i k & 0 & \frac{\partial}{\partial y} \\
-\frac{\partial}{\partial x} & -\frac{\partial}{\partial y} & 0
\end{array}\right), \\
d_{1}=\left(\begin{array}{ccc}
\left(\varepsilon^{-1}\right)_{11} & \left(\varepsilon^{-1}\right)_{12} & 0 \\
\left(\varepsilon^{-1}\right)_{12}^{*} & \left(\varepsilon^{-1}\right)_{22} & 0 \\
0 & 0 & \mu_{33}
\end{array}\right), \quad d_{2}=\left(\begin{array}{ccc}
\left(\mu^{-1}\right)_{11} & \left(\mu^{-1}\right)_{12} & 0 \\
\left(\mu^{-1}\right)_{12}^{*} & \left(\mu^{-1}\right)_{22} & 0 \\
0 & 0 & \varepsilon_{33}
\end{array}\right), \\
A_{1}=\left(B_{x}, B_{y}, E_{z}\right)^{T}, \quad A_{2}=\left(D_{x}, D_{y}, H_{z}\right)^{T}, \\
J=\frac{4 \pi}{c}\left(-j_{y}, j_{x},-c \rho\right) .
\end{gathered}
$$

Запишем в обозначениях (1.10)-(1.13) уравнения (1.6), (1.7):

$$
\begin{gathered}
a_{1} A_{1}=d_{1} \frac{\partial}{\partial z} A_{2}, \\
a_{2} A_{2}=d_{2} \frac{\partial}{\partial z} A_{1}+J .
\end{gathered}
$$

Далее рассмотрим постановку краевой задачи в цилиндре $Q$ для уравнений (1.14), (1.15). 


\section{§ 2. Спектральная задача теории регулярных волноводов}

Вне области, занятой токами и зарядами, векторы $A_{1}, A_{2}$ удовлетворяют однородным уравнениям

$$
\begin{aligned}
& a_{1} A_{1}=d_{1} \frac{\partial A_{2}}{\partial z}, \\
& a_{2} A_{2}=d_{2} \frac{\partial A_{1}}{\partial z} .
\end{aligned}
$$

Поставим спектральную задачу в сечении $\Omega$ цилиндра $Q$ для уравнений

$$
\begin{aligned}
& a_{1} A_{1}=i \gamma d_{1} A_{2}, \\
& a_{2} A_{2}=i \gamma d_{2} A_{1} .
\end{aligned}
$$

Эта спектральная задача возникает при поиске решений системы (2.1), (2.2) вида

$$
A_{1}=A_{1}(x, y) e^{i \gamma z}, \quad A_{2}=A_{2}(x, y) e^{i \gamma z} .
$$

ЗАмЕчАНИЕ 2.1. Поскольку система уравнений (2.3), (2.4) не является симметричной, в случае кратных собственных значений, помимо собственных векторов, могут существовать присоединенные векторы задачи (2.3), (2.4); (1.3), (1.4). Пусть $\gamma_{n}$ - собственное значение кратности $N$, которому соответствуют $N-1$ присоединенных векторов, $A_{1_{n, 1}}, A_{2_{n, 1}}$ - соответствующие ему собственные векторы. Система уравнений для присоединенных векторов имеет вид

$$
\begin{aligned}
& a_{1} A_{1_{n, k+1}}=i \gamma_{n} d_{1} A_{2_{n, k+1}}+d_{1} A_{2_{n, k}}, \\
& a_{2} A_{2_{n, k+1}}=i \gamma_{n} d_{2} A_{1_{n, k+1}}+d_{2} A_{1_{n, k}},
\end{aligned}
$$

где $k=1, \ldots, N-1$. Собственные и присоединенные векторы называют корневыми векторами [4].

ЗАмечание 2.2. Решениям спектральной задачи $\left(\gamma_{n}, A_{i_{n, k}}\right)$, где $\gamma_{n}$ - собственные значения, $A_{i_{n, k}}, i=1,2$, - собственные и присоединенные векторы, отвечают решения однородных уравнений (2.1), (2.2) вида

$$
A_{i}=\sum_{k=1}^{N} \frac{z^{N-k}}{(N-k) !} e^{ \pm i \gamma_{n} z} A_{i_{n, k}}(x, y) .
$$

Решения вида $e^{i \gamma_{n} z} A_{i_{n, 1}}(x, y)$ будем называть, следуя определению, введенному П.Е. Краснушкиным, нормальными волнами [4], [8], а вида $z^{N-k} e^{i \gamma_{n} z} \times$ $A_{i_{n, k}}(x, y)$ - присоединенными волнами.

ЗАмЕЧАНИЕ 2.3. Будем предполагать вплоть до введения определения обобщенного решения (определение 2.1), что векторы $E, H, B, D$ и коэффициенты $\varepsilon, \mu$ обладают необходимой гладкостью для выполнения требуемых операций дифференцирования. Цель следующих утверждений 2.1-2.5 заключается в доказательстве эквивалентности постановки спектральных задач для уравнений $(2.3),(2.4)$ относительно векторов $A_{1}, A_{2}$ и спектральной задачи для однородной системы уравнений (1.1), (1.2). 
УтвеРЖДЕНИЕ 2.1. Решения системы уравнений (2.3)-(2.6), отвечающие ненулевым собственным значениям $\gamma_{n}$, удовлетворяют дополнительным дифференциалъным уравнениям (1.8), (1.9) при $j_{z}$, равном нулю.

В справедливости утверждения 2.1 убеждаемся непосредственной проверкой.

Будем для краткости рассматривать уравнения только для собственных векторов, причем индексы у собственных векторов и собственных значений не будем выписывать в тех случаях, когда это возможно для сокращения обозначений.

Для постановки спектральной задачи необходимо дополнить систему уравнений (2.3)-(2.6) граничными условиями. Напомним, что собственные значения $\gamma$ не равны нулю, поскольку $k$ не совпадает с частотой отсечки. В качестве граничных условий для векторов $A_{1}, A_{2}$ достаточно использовать граничные условия только для вектора $A_{1}$ :

$$
\left.B \boldsymbol{n}\right|_{\partial \Omega}=0,\left.\quad E_{z}\right|_{\partial \Omega}=0 .
$$

Легко проверить, что справедливо

УтвЕРЖДЕНИЕ 2.2. Граничные условия $E \times\left.\boldsymbol{n}\right|_{\partial \Omega}=0$ при $\gamma \neq 0$ являются следствиями условий (2.7).

Аналогично, в качестве условий сопряжения на линиях разрыва $S$ диэлектрической и магнитной проницаемостей достаточно задать условия

$$
\begin{array}{ll}
{\left.[B \boldsymbol{n}]\right|_{S}=0,} & {\left.\left[E_{z}\right]\right|_{S}=0,} \\
{\left.[D \boldsymbol{n}]\right|_{S}=0,} & {\left.\left[H_{z}\right]\right|_{S}=0,}
\end{array}
$$

которые являются основными. (Учитывая, что матрицы диэлектрической и магнитной проницаемостей $\varepsilon$ и $\mu$ не зависят от координаты $z$, мы обозначаем линии разрыва через $S$ так же, как и поверхности разрыва.)

Справедливо

УТВЕРЖДЕНИЕ 2.3. Условия $\left.[H \times \boldsymbol{n}]\right|_{S}=0,\left.[E \times \boldsymbol{n}]\right|_{S}=0$ являются следствиями уравнений (2.3), (2.4) и основных условий сопряжения.

Справедливость утверждений 2.2, 2.3 устанавливается непосредственной проверкой.

Систему (2.3)-(2.6) при ненулевых $\gamma_{n}$ можно свести к уравнению либо относительно вектора $A_{1}$, либо относительно вектора $A_{2}$.

Система уравнений для собственных и присоединенных векторов $A_{1}$ имеет вид

$$
\begin{gathered}
a_{2} d_{1}^{-1} a_{1} A_{1_{n, 1}}=-\gamma_{n}^{2} d_{2} A_{1_{n, 1}}, \\
a_{2} d_{1}^{-1} a_{1} A_{1_{n, 2}}=-\gamma_{n}^{2} d_{2} A_{1_{n, 2}}+2 i \gamma_{n} d_{2} A_{2_{n, 1}}, \\
a_{2} d_{1}^{-1} a_{1} A_{1_{n, k+2}}=-\gamma_{n}^{2} d_{2} A_{1_{n, k+2}}+2 i \gamma_{n} d_{2} A_{1_{n, k+1}}+d_{2} A_{1_{n, k}} .
\end{gathered}
$$

Систему уравнений для векторов $A_{2}$ в силу их аналогичного вида не будем приводить.

Необходимо дополнить уравнения (2.8)-(2.10) граничными условиями и условиями сопряжения, следующими из условий (1.3), (1.4). 
Для сокращения записи введем следующие обозначения:

$$
\begin{aligned}
& B_{\perp}=\left(B_{x}, B_{y}\right), \quad \mathcal{J}=\left(\begin{array}{rr}
0 & 1 \\
-1 & 0
\end{array}\right) \\
& \operatorname{div} B_{\perp}=\frac{\partial B_{x}}{\partial x}+\frac{\partial B_{y}}{\partial y}, \quad \operatorname{rot} B_{\perp}=\frac{\partial B_{y}}{\partial x}-\frac{\partial B_{x}}{\partial y}, \\
& \operatorname{grad} \phi=\frac{\partial \phi}{\partial x} e_{x}+\frac{\partial \phi}{\partial y} e_{y}, \quad \operatorname{rot} \phi=\frac{\partial \phi}{\partial y} e_{x}-\frac{\partial \phi}{\partial x} e_{y},
\end{aligned}
$$

где $\varepsilon, \mu$ - подматрицы матриц $\varepsilon_{i j}, \mu_{i j}, i, j=1,2$.

В обозначениях (2.11), (2.12) уравнение (2.8) принимает вид

$$
\begin{gathered}
-\operatorname{grad} \mu_{33}^{-1} \operatorname{div} B_{\perp}+k^{2} \mathcal{J} \varepsilon \mathcal{J} B_{\perp}-i k \mathcal{J} \varepsilon \operatorname{grad} E_{z}=-\gamma^{2} \mu^{-1} B_{\perp}, \\
-i k \operatorname{div}\left(\varepsilon \mathcal{J} B_{\perp}\right)-\operatorname{div} \varepsilon \operatorname{grad}_{\perp} E_{z}=-\gamma^{2} \varepsilon_{33} E_{z} .
\end{gathered}
$$

Дополним уравнения (2.13), (2.14) краевыми условиями

$$
\left.B \boldsymbol{n}\right|_{\partial \Omega}=0,\left.\quad E_{z}\right|_{\partial \Omega}=0
$$

и условиями сопряжения, принимающими вид

$$
\begin{gathered}
{\left.[B \boldsymbol{n}]\right|_{S}=0,\left.\quad\left[E_{z}\right]\right|_{S}=0,} \\
{\left.\left[\mu_{33}^{-1} \operatorname{div} B_{\perp}\right]\right|_{S}=0,\left.\quad\left[\varepsilon\left(\operatorname{grad} E_{z}+i k\left(B \times e_{z}\right)\right) \boldsymbol{n}\right]\right|_{S}=0,}
\end{gathered}
$$

где $\boldsymbol{n}$ - нормаль к границе $\partial \Omega$ или линии разрыва $S$.

В качестве "условий на ребре", учитывая наличие угловых точек границы $\partial \Omega$, потребуем выполнения следующих условий:

$$
B_{\perp} \in L_{2}(\Omega), \quad \operatorname{div} B_{\perp} \in L_{2}(\Omega), \quad E_{z} \in H^{1}(\Omega) .
$$

УТВЕРЖДЕНИЕ 2.4. Условия сопряљения (2.17) эквивалентны условиям

$$
\left.[D \boldsymbol{n}]\right|_{S}=0,\left.\quad\left[H_{z}\right]\right|_{S}=0,
$$

выраженным через вектор $\left(B_{\perp}, E_{z}\right)^{T}$.

УТВЕРЖДЕНИЕ 2.5. Пусть $A_{1_{n}}$ - собственный вектор задачи (2.13)-(2.17). Тогда векторы $A_{1_{n}}$ и $A_{2_{n}}=-i \frac{1}{\gamma_{n}} d_{1}^{-1} a_{1} A_{1_{n}}$ являются решением задачи (2.3), (2.4), (2.7).

При этом основные граничные условия и условия сопряжения выполняются в силу соотношений (1.12), а дополнительные условия, как было показано, являются их следствиями.

Таким образом, задача $(2.3),(2.4),(2.7)$ и задача (2.13)-(2.17) имеют одни и те же собственные значения. Зная собственные векторы задачи (2.13)-(2.17), можно определить собственные векторы задачи (2.3), (2.4), (2.7). Для присоединенных векторов могут быть проведены аналогичные выкладки. Таким же образом исходная задача может быть сведена к задаче подобного вида относительно вектора $A_{2}$.

Рассмотрим спектральную задачу (2.13)-(2.17) и найдем её обобщенные решения. 
ЗАмЕчАниЕ 2.4. В настоящей работе рассматриваются обобщенные решения задачи (2.13)-(2.17). Для введения понятия обобщенного решения и его дальнейшего исследования требование кусочной непрерывности коэффициентов $\varepsilon, \mu$ является, очевидно, излишним. Как известно [23], достаточно было бы потребовать, чтобы коэффициенты системы уравнений были измеримыми ограниченными функциями и выполнялись условия

$$
\begin{gathered}
\alpha \sum_{i=1}^{3}\left|\xi_{i}\right|^{2} \leqslant \sum_{i, j=1}^{3} \varepsilon_{i j} \xi_{i} \xi_{j}^{*} \leqslant \beta \sum_{i=1}^{3}\left|\xi_{i}\right|^{2}, \\
\alpha \sum_{i=1}^{3}\left|\xi_{i}\right|^{2} \leqslant \sum_{i, j=1}^{3} \mu_{i j} \xi_{i} \xi_{j}^{*} \leqslant \beta \sum_{i=1}^{3}\left|\xi_{i}\right|^{2} \quad \forall \xi \in \mathbb{C}^{3},
\end{gathered}
$$

где $\alpha>0, \beta>0$. В возникающих в практических приложениях задачах электродинамики волноводов коэффициенты $\varepsilon, \mu$ обладают определенной гладкостью. В качестве характерного поведения коэффициентов $\varepsilon, \mu$ можно рассматривать следующий частный случай. Область $\Omega_{1}$ с границей класса $C^{\infty}$ является подобластью области $\Omega$, т. е. $\Omega_{1} \subset \Omega$, причем граница области $\Omega_{1}$ не имеет общих точек с границей области $\Omega$. Обозначим $\Omega_{2}=\Omega \backslash \Omega_{1}$. Коэффициенты $\varepsilon, \mu$ системы уравнений обладают сколь угодно высокой степенью гладкости в замкнутых областях $\bar{\Omega}_{1}$ и $\bar{\Omega}_{2}$, т. е. $\varepsilon_{i j} \in C^{n}\left(\bar{\Omega}_{1}\right), \varepsilon_{i j} \in C^{n}\left(\bar{\Omega}_{2}\right)$, $\mu_{i j} \in C^{n}\left(\bar{\Omega}_{1}\right), \mu_{i j} \in C^{n}\left(\bar{\Omega}_{2}\right)$, где $n$ - сколь угодно велико. В работе [20] доказано, что гладкость решений системы уравнений Максвелла имеет тот же характер, что и для систем эллиптических уравнений, вблизи гладких участков границы и в области гладкости коэффициентов уравнений. (В окрестности угловых точек границы решение может быть неограниченным.) Мы не будем рассматривать вопрос о связи гладкости коэффициентов системы уравнений с гладкостью решения. Исследование этого вопроса сводится к применению результатов работы [20]. (Считаем, что граница цилиндра $Q$ принадлежит классу границ, описанных в $[19, \S 5$, п. 5.3].)

Предположим, что $A$ - классическое решение задачи (2.13)-(2.17). Обозначим $A_{\perp}=\left(A_{x}, A_{y}\right)$. Умножим систему $(2.13),(2.14)$ на произвольный вектор $\widetilde{A}$ (непрерывно дифференцируемый в областях гладкости коэффициентов вплоть до гладких участков границы и удовлетворяющий граничным условиям, условиям сопряжения (2.15)-(2.17) и условиям на ребре) и проинтегрируем по частям:

$$
\begin{aligned}
& \int_{\Omega}(- \widetilde{A}_{\perp} \operatorname{grad} \mu_{33}^{-1} \operatorname{div} A_{\perp}+k^{2} \widetilde{A}_{\perp} \mathcal{J} \varepsilon \mathcal{J} A_{\perp}-i k \widetilde{A}_{\perp} \mathcal{J} \varepsilon \operatorname{grad} A_{z} \\
&\left.\quad-i k \widetilde{A}_{z} \operatorname{div} \varepsilon \mathcal{J} A_{\perp}-\widetilde{A}_{z} \operatorname{div} \varepsilon \operatorname{grad} A_{z}\right) d S \\
&= \int_{\Omega}\left(\mu_{33}^{-1} \operatorname{div} A_{\perp} \operatorname{div} \widetilde{A}_{\perp}+k^{2} \widetilde{A}_{\perp} \mathcal{J} \varepsilon \mathcal{J} A_{\perp}-i k \widetilde{A}_{\perp} \mathcal{J} \varepsilon \operatorname{grad} A_{z}\right. \\
&\left.\quad+i k \varepsilon \mathcal{J} A_{\perp} \operatorname{grad} \widetilde{A}_{z}-\varepsilon \operatorname{grad} A_{z} \operatorname{grad} \widetilde{A}_{z}\right) d S \\
&=-\gamma^{2} \int_{\Omega}\left(\mu^{-1} A_{\perp} \widetilde{A}_{\perp}+\varepsilon_{33} A_{z} \widetilde{A}_{z}\right) d S .
\end{aligned}
$$

Решение задачи (2.13)-(2.17) является решением полученного уравнения при любом достаточно гладком $\widetilde{A}$, удовлетворяющем граничным условиям и усло- 
виям сопряжения. Для того чтобы сформулировать обобщенную постановку задачи, напомним определения некоторых функциональных пространств.

Введем функциональное пространство (см. [3], [27], [19])

$$
H(\operatorname{div}, \Omega)=\left\{A_{\perp} \in\left(L_{2}(\Omega)\right)^{2}, \operatorname{div} A_{\perp} \in L_{2}(\Omega)\right\},
$$

которое является гильбертовым относительно нормы $\left\|A_{\perp}\right\|_{H(\mathrm{div})}^{2}=\left\|A_{\perp}\right\|_{L_{2}}^{2}+$ $\left\|\operatorname{div} A_{\perp}\right\|_{L_{2}}^{2}$. Операция $\operatorname{div}$ понимается в смысле распределений.

Введем пространство $H_{0}(\operatorname{div}, \Omega)=\left\{A_{\perp} \in H(\operatorname{div}, \Omega),\left.A_{\perp} \boldsymbol{n}\right|_{\partial \Omega}=0\right\}$, где под условием $\left.A_{\perp} \boldsymbol{n}\right|_{\partial \Omega}=0$ понимаем выполнение равенства

$$
\left(\operatorname{div} A_{\perp}, \phi\right)_{L_{2}}=-\left(A_{\perp}, \operatorname{grad} \phi\right)_{L_{2}} \quad \forall \phi \in H^{1}(\Omega) .
$$

Введем пространство $W=H_{0}(\operatorname{div}, \Omega) \oplus \dot{H}^{1}(\Omega)$ (т. е. пространство векторов $\left.A_{\perp} \in H_{0}(\mathrm{div}), A_{z} \in \dot{H}^{1}\right)$, которое является гильбертовым относительно нормы

$$
\|A\|_{W}^{2}=\left\|A_{\perp}\right\|_{H(\operatorname{div})}^{2}+\left\|A_{z}\right\|_{H^{1}}^{2} .
$$

Введем в пространстве $W$ полуторалинейные формы

$$
\begin{gathered}
a(A, \widetilde{A})=\left(\mu_{33}^{-1} \operatorname{div} A_{\perp}, \operatorname{div} \widetilde{A}_{\perp}\right)_{L_{2}}+\left(\varepsilon \operatorname{grad} A_{z}, \operatorname{grad} \widetilde{A}_{z}\right)_{L_{2}}, \\
b(A, \widetilde{A})=k^{2}\left(\mathcal{J} \varepsilon \mathcal{J} A_{\perp}, \widetilde{A}_{\perp}\right)_{L_{2}}-i k\left(\mathcal{J} \varepsilon \operatorname{grad} A_{z}, \widetilde{A}_{\perp}\right)_{L_{2}}+i k\left(\varepsilon \mathcal{J} A_{\perp}, \operatorname{grad} \widetilde{A}_{z}\right)_{L_{2}} \\
c(A, \widetilde{A})=\left(\mu^{-1} A_{\perp}, \widetilde{A}_{\perp}\right)_{L_{2}}+\left(\varepsilon_{33} A_{z}, \widetilde{A}_{z}\right)_{L_{2}} .
\end{gathered}
$$

Будем искать обобщенное решение задачи (2.13)-(2.17) в пространстве $W$ (при этом ограничиваемся записью только уравнения для собственных функций).

ОПРЕДЕЛЕНИЕ 2.1. Обобщенным решением задачи (2.13)-(2.17) будем называть ненулевой вектор $A \in W$ и комплексное число $\gamma$ такие, что выполняется уравнение

$$
a(A, \widetilde{A})+b(A, \widetilde{A})=-\gamma^{2} c(A, \widetilde{A}) \quad \forall \widetilde{A} \in W .
$$

Отметим следующую особенность задачи (2.21), рассматриваемой в пространстве $W$. Справедливо проверяемое непосредственной подстановкой

УтвеРЖДЕНИЕ 2.6. Любой вектор вида $(\operatorname{rot} \phi, i k \phi)^{T}$, где $\phi \in \dot{H}^{1}(\Omega)$, является собственным вектором задачи (2.21), отвечающим нулевому собственному значению.

Таким образом, задача (2.21) имеет бесконечномерное ядро.

В то же время справедливо

УТВЕРЖДЕНИЕ 2.7. Корневые векторы задачи, отвечающие ненулевым собственным значениям $\gamma_{n}^{2}$, удовлетворяют уравнению

$$
\left(\mu^{-1} A_{\perp}, \operatorname{rot} \phi\right)_{L_{2}}+i k\left(\varepsilon_{33} A_{z}, \phi\right)_{L_{2}}=0 \quad \forall \phi \in \dot{H}^{1}(\Omega) .
$$

Данное утверждение проверяется подстановкой в уравнение (2.21) вектора $\widetilde{A}=(\operatorname{rot} \phi,-i k \phi)$ для любого $\phi \in \dot{H}^{1}(\Omega)$.

ЗАмЕчАниЕ 2.5. Уравнение (2.22) представляет собой обобщенную форму одного из уравнений Максвелла, не использованного при постановке задачи. 
Введем функциональное пространство

$$
V=\left\{A \in W,\left(\mu^{-1} A_{\perp}, \operatorname{rot} \phi\right)_{L_{2}}+i k\left(\varepsilon_{33} A_{z}, \phi\right)_{L_{2}}=0 \quad \forall \phi \in \dot{H}^{1}\right\} .
$$

Будем рассматривать далее спектральную задачу в пространстве $V$. Норма пространства $V$ определяется так же, как норма пространства $W$.

Справедливо легко проверяемое

\section{УТВЕРЖДЕНИЕ 2.8. Пространство $V$ является полным.}

Перейдем теперь к исследованию вопроса о полноте системы корневых векторов задачи (2.21). Напомним

ОПРедЕЛЕНиЕ 2.2. Система векторов $A_{n}$ называется полной в некотором пространстве $V$, если замыкание линейной оболочки векторов $A_{n}$ совпадает c $V[9]$.

Полуторалинейная форма $a(A, \widetilde{A})$ определяет в гильбертовом пространстве $V$ скалярное произведение

$$
(A, \widetilde{A})_{V}=a(A, \widetilde{A}) .
$$

В силу ограниченности и положительной определенности матриц $\varepsilon, \mu$ формы $b(A, \widetilde{A}), c(A, \widetilde{A})$ удовлетворяют условиям

$$
\begin{gathered}
|b(A, \widetilde{A})| \leqslant N\left(\left\|B_{\perp}\right\|_{L_{2}}\left\|\widetilde{B}_{\perp}\right\|_{L_{2}}+\left\|E_{z}\right\|_{\dot{H}^{1}}\left\|\widetilde{B}_{\perp}\right\|_{L_{2}}+\left\|B_{\perp}\right\|_{L_{2}}\left\|\widetilde{E}_{z}\right\|_{H^{1}}\right), \\
|c(A, \widetilde{A})| \leqslant N\|A\|_{L_{2}}\|\widetilde{A}\|_{L_{2}},
\end{gathered}
$$

где $N$ - некоторая константа. По теореме Рисса [28] существуют линейные ограниченные операторы $C, H$ такие, что

$$
\begin{aligned}
& (C A, \widetilde{A})_{V}=b(A, \widetilde{A}), \\
& (H A, \widetilde{A})_{V}=c(A, \widetilde{A}) .
\end{aligned}
$$

Дальнейшее изложение существенно опирается на компактность вложения пространства $V$ в $\left(L_{2}\right)^{3}$. Для доказательства компактности вложения понадобится аналог теоремы о разложении произвольного векторного поля из $L_{2}$ на вихревую и потенциальную части [27].

ТеОрема 2.1. Для любого вектора $A \in L_{2}(\Omega)$ справедливо представление в следующем виде:

$$
A=\mu \operatorname{grad}_{\perp} \psi+\operatorname{rot}_{\perp} \chi
$$

где $\psi \in H^{1}, \chi \in \dot{H}^{1}$.

ДокАЗАТЕЛЬСтво. Определим функцию $\psi \in H^{1}$ как решение уравнения

$$
(\mu \operatorname{grad} \psi, \operatorname{grad} \phi)_{L_{2}}=(A, \operatorname{grad} \phi)_{L_{2}} \quad \forall \phi \in H^{1} .
$$

Поскольку вектор $\bar{A}=\mu \operatorname{grad} \psi-A$ удовлетворяет условию

$$
(\bar{A}, \operatorname{grad} \phi)_{L_{2}}=0 \quad \forall \phi \in H^{1},
$$

существует $\chi \in \dot{H}^{1}$ такое, что $\bar{A}=\operatorname{rot} \chi[27]$. 
Теорема 2.2. Пространство $V$ компактно вложено в $\left(L_{2}\right)^{3}$.

ДоказАтельство. Рассмотрим последовательность $A_{n}=\left(B_{\perp_{n}}, E_{z_{n}}\right)$ векторов, ограниченных в пространстве $V$ :

$$
\left\|B_{\perp_{n}}\right\|_{H_{0}(\operatorname{div}, \Omega)}+\left\|E_{z_{n}}\right\|_{\dot{H}^{1}}<C .
$$

Поскольку последовательность $E_{z_{n}}$ ограничена в $H^{1}$, из нее можно выделить подпоследовательность, сходящуюся сильно в $L_{2}$. Согласно теореме 2.1 векторы $B_{\perp_{n}}$ допускают представление в виде

$$
B_{\perp_{n}}=\mu \operatorname{grad} \psi_{n}+\operatorname{rot} \chi_{n}
$$

В силу того, что $\psi_{n}$ удовлетворяют уравнению

$$
\left(\mu \operatorname{grad} \psi_{n}, \operatorname{grad} \phi\right)_{L_{2}}=\left(\operatorname{div} B_{\perp_{n}}, \phi\right)_{L_{2}} \quad \forall \phi \in H^{1}
$$

и последовательность $\operatorname{div} B_{\perp_{n}}$ ограничена в $L_{2}$, из последовательности $\psi_{n}$ можно выделить подпоследовательность, сходящуюся сильно в $H^{1}$. Таким образом, из последовательности $\mu \operatorname{grad} \psi_{n}$ можно выделить подпоследовательность, сходящуюся сильно в $\left(L_{2}\right)^{2}$.

Поскольку векторы $B_{\perp_{n}}$ пространства $V$ удовлетворяют уравнению

$$
\left(\mu^{-1} B_{\perp_{n}}, \operatorname{rot} \phi\right)_{L_{2}}=-i k\left(\varepsilon_{33} E_{z_{n}}, \phi\right)_{L_{2}} \quad \forall \phi \in \dot{H}^{1}
$$

то $\chi_{n}$ удовлетворяет уравнению

$$
\left(\mu^{-1} \operatorname{rot} \chi_{n}, \operatorname{rot} \phi\right)_{L_{2}}=-i k\left(\varepsilon_{33} E_{z_{n}}, \phi\right)_{L_{2}} \quad \forall \phi \in \dot{H}^{1} .
$$

Перепишем уравнение (2.29) в виде

$$
\left(a_{i j} \frac{\partial \chi_{n}}{\partial x_{i}}, \frac{\partial \phi}{\partial x_{j}}\right)_{L_{2}}=-i k\left(\varepsilon_{33} E_{z_{n}}, \phi\right)_{L_{2}} \quad \forall \phi \in \dot{H}^{1},
$$

где $a_{i i}=\left(\mu^{-1}\right)_{i i}, a_{i j}=-\left(\mu^{-1}\right)_{i j}, i \neq j, i, j=1,2$. Так как матрица $a_{i j}$ положительно определена, то в силу ограниченности последовательности $E_{z_{n}}$ в $\dot{H}^{1}$ из последовательности $\chi_{n}$ можно выделить подпоследовательность, сходящуюся сильно в $\dot{H}^{1}$. Следовательно, соответствующая подпоследовательность последовательности rot $\chi_{n}$ сходится в $\left(L_{2}\right)^{2}$ сильно. Таким образом, утверждение теоремы доказано.

В силу неравенств $(2.23),(2.24)$ и компактности вложения $V$ в $\left(L_{2}\right)^{3}$ операторы $C$ и $H$ компактны. Поскольку уравнение $(2.21)$ справедливо для любого $\widetilde{A} \in V$, задача (2.21) эквивалентна в пространстве $V$ спектральной задаче для операторного пучка

$$
M(\gamma)=(I+C) A+\gamma^{2} H A
$$

Операторы $C$ и $H$ компактны, оператор $H$ самосопряжен и аннулируется только в нуле, т. е. из условия $H A=0$ следует $A=0$. Докажем, что справедлива

Теорема 2.3. Оператор $H$ является оператором Гилъберта-Шмидта, m.е. $\sum_{k} \nu_{k}^{2}<\infty$, где $\nu_{k}$ - собственные значения оператора $H$. 
ДокАЗАтельство. Собственные значения оператора $H$, определенного равенством (2.26), являются обратными к собственным значениям $\lambda$ задачи

$$
a(A, \widetilde{A})=\lambda c(A, \widetilde{A}) \quad \forall \widetilde{A} \in V,
$$

или в покомпонентной записи:

$$
\begin{aligned}
& \left(\mu_{33}^{-1} \operatorname{div} B_{\perp}, \operatorname{div} \widetilde{B}_{\perp}\right)_{L_{2}}+\left(\varepsilon \operatorname{grad} E_{z}, \operatorname{grad} \widetilde{E}_{z}\right)_{L_{2}} \\
& \quad=\lambda\left(\left(\mu^{-1} B_{\perp}, \widetilde{B}_{\perp}\right)_{L_{2}}+\left(\varepsilon_{33} E_{z}, \widetilde{E}_{z}\right)_{L_{2}}\right) \quad \forall\left(\widetilde{B}_{\perp}, \widetilde{E}_{z}\right) \in V .
\end{aligned}
$$

Рассмотрим решения задачи (2.31) вида

$$
B_{\perp}=\mu \operatorname{grad} \psi, \quad E_{z}=0,
$$

и рассмотрим задачу на собственные значения:

$$
(\mu \operatorname{grad} \psi, \operatorname{grad} \phi)=\lambda\left(\mu_{33} \psi, \phi\right) \quad \forall \phi \in H^{1} .
$$

Решения задачи (2.32) удовлетворяют уравнению

$$
-\operatorname{div} \mu \operatorname{grad} \psi_{n}=\lambda_{n} \mu_{33} \psi_{n} .
$$

Векторы $B_{\perp_{n}}=\mu \operatorname{grad} \psi_{n}$ являются решением задачи $(2.31)$, поскольку

$$
\left(\mu_{33}^{-1} \operatorname{div} \mu \operatorname{grad} \psi_{n}, \operatorname{div} \mu \operatorname{grad} \widetilde{\psi}\right)_{L_{2}}=\lambda_{n}\left(\mu^{-1} \operatorname{grad} \psi_{n}, \operatorname{grad} \widetilde{\psi}\right)_{L_{2}} .
$$

Собственные функции $\psi_{n}$ задачи (2.32) полны в пространстве $H^{1}$ и удовлетворяют условиям ортогональности:

$$
\left(\mu \operatorname{grad} \psi_{n}, \operatorname{grad} \psi_{m}\right)_{L_{2}}=0, \quad n \neq m .
$$

Собственные значения $\lambda_{n}$ задачи (2.33) имеют асимптотику $O(n)$.

Пусть $\left(B_{\perp}, E_{z}\right)$ - собственный вектор. Учитывая то, что $\psi_{n}$ образуют полную систему в пространстве $H^{1}$, и ортогональность собственных векторов задачи (2.31), для которых имеет место равенство $B_{\perp}=\mu \operatorname{grad} \psi+\operatorname{rot} \chi$, получим $B_{\perp}=\operatorname{rot} \chi$.

Уравнение (2.31) имеет вид

$$
\left(\varepsilon \operatorname{grad} E_{z}, \operatorname{grad} \widetilde{E}_{z}\right)_{L_{2}}=\lambda\left(\left(\varepsilon_{33} E_{z}, \widetilde{E}_{z}\right)_{L_{2}}+\left(\mu^{-1} B_{\perp}, \widetilde{B}_{\perp}\right)_{L_{2}}\right) \quad \forall \widetilde{A} \in V .
$$

Вектор $\widetilde{B}_{\perp}$ представим в виде $\widetilde{B}_{\perp}=\mu \operatorname{grad} \widetilde{\psi}+\operatorname{rot} \widetilde{\chi}$. Уравнение $(2.34)$ принимает вид

$$
\left(\varepsilon \operatorname{grad} E_{z}, \operatorname{grad} \widetilde{E}_{z}\right)_{L_{2}}=\lambda\left(\left(\varepsilon_{33} E_{z}, \widetilde{E}_{z}\right)_{L_{2}}+\left(\mu^{-1} \operatorname{rot} \chi, \operatorname{rot} \widetilde{\chi}\right)_{L_{2}}\right) .
$$

Поскольку $\chi \in \dot{H}^{1}$ удовлетворяет уравнению

$$
\left(\mu^{-1} \operatorname{rot} \chi, \operatorname{rot} \tilde{\chi}\right)_{L_{2}}=i k\left(\varepsilon_{33} E_{z}, \widetilde{\chi}\right)_{L_{2}} \quad \forall \tilde{\chi} \in \dot{H}^{1},
$$

в силу теоремы Рисса существует ограниченный самосопряженный оператор $Y$ такой, что $\chi=i k Y \varepsilon_{33} E_{z}$. Аналогично, поскольку $\widetilde{\chi}$ удовлетворяет задаче того же вида, то $\widetilde{\chi}=i k Y \varepsilon_{33} \widetilde{E}_{z}$. 
В результате имеем

$$
\left(\mu^{-1} B_{\perp}, \widetilde{B}_{\perp}\right)_{L_{2}}=k^{2}\left(\varepsilon_{33} E_{z}, Y \varepsilon_{33} \widetilde{E}_{z}\right)_{L_{2}} .
$$

Таким образом, уравнение (2.34) принимает вид

$$
\left(\varepsilon \operatorname{grad} E_{z}, \operatorname{grad} \widetilde{E}_{z}\right)_{L_{2}}=\lambda\left(\left(\varepsilon_{33} E_{z}, \widetilde{E}_{z}\right)_{L_{2}}+k^{2}\left(\varepsilon_{33} E_{z}, Y \varepsilon_{33} \widetilde{E}_{z}\right)_{L_{2}}\right) .
$$

Собственные значения задачи (2.34), как следует из оценок, получающихся применением принципа минимакса [29], имеют асимптотику $O(n)$. Следовательно, $H$ - оператор Гильберта-Шмидта.

Таким образом, операторный пучок $M(\gamma)$ удовлетворяет теореме Келдыша о полноте корневых векторов операторного пучка [30], [31]. Напомним её формулировку применительно к рассматриваемой задаче.

ТЕОРЕма КЕЛДЫША. Пусть $I+C+\gamma^{2} H$ - операторный пучок, где операторы $C$ и $H$ компактны, $H$ - полный самосопряженный оператор конечного порядка. Тогда система корневых векторов операторного пучка полна в пространстве $V$. Пусть $I+C+\gamma A_{1} H^{\frac{1}{2}}+\gamma^{2} H$ - операторный пучок, где операторы $C, A_{1}, H^{\frac{1}{2}}$ компактны, $H^{\frac{1}{2}}$ - полный самосопряженный оператор конечного порядка. Тогда система корневых векторов операторного пучка двукратно полна в пространстве $V$.

Корневые векторы операторного пучка $M(\gamma)$ удовлетворяют уравнениям (2.8)-(2.10) для корневых векторов квадратичного операторного пучка относительно параметра $\gamma$. Поскольку в операторный пучок входит только член с $\gamma^{2}$, то можно рассматривать задачу о корневых векторах линейного операторного пучка относительно спектрального параметра $\gamma^{2}$. Следовательно, в силу теоремы Келдыша справедлива следующая

Теорема 2.4. Система корневых векторов задачи (2.21) полна в пространстве $V$.

В силу теоремы Келдыша о спектре операторного пучка справедливы следующие утверждения.

УтВЕРЖДЕНИЕ 2.9. Все собственные значения, кроме, быть может, конечного числа, лежат в секторе $\left|\arg \left(\gamma_{\max }^{2}-\gamma^{2}\right)\right|<\beta$, где $\beta$ сколь угодно мало, $\gamma_{\max }^{2}-$ некоторая константа.

УТВЕРЖДЕНИЕ 2.10. Квадраты постоянных распространения $\gamma_{n}^{2}$ имеют асимптотику $O(n), n \rightarrow \infty$.

Из утверждения 2.10 следует, что справедливо

УтВЕРЖДЕНИЕ 2.11. Существует лищь конечное число вещественных положительных собственных значений $\gamma_{n}^{2}$.

Половина системы корневых векторов квадратичного пучка, выбираемая стандартным образом, полна в $V$.

Будем считать, что вещественным значениям $\gamma_{n}$ соответствуют только собственные векторы, а присоединенные векторы отсутствуют. Несложно выписать биортогональную систему к системе корневых векторов $A_{1_{n, k}}$. В качестве биортогональной системы выберем систему корневых векторов задачи, сопряженной к задаче (2.13)-(2.17). 
Отметим, что после умножения системы уравнений (2.13), (2.14) на матрицу $\nu=\left(\begin{array}{cc}1 & 0 \\ 0 & -1\end{array}\right)$ система принимает симметричный вид:

$$
\left(\begin{array}{cc}
-\operatorname{grad} \mu_{33}^{-1} \operatorname{div}+k^{2} \mathcal{J} \varepsilon \mathcal{J} & -i k \mathcal{J} \varepsilon \operatorname{grad} \\
i k \operatorname{div} \varepsilon \mathcal{J} & \operatorname{div} \varepsilon \operatorname{grad}
\end{array}\right)\left(\begin{array}{c}
B_{\perp} \\
E_{z}
\end{array}\right)=-\gamma^{2}\left(\begin{array}{cc}
\mu^{-1} & 0 \\
0 & -\varepsilon_{33}
\end{array}\right)\left(\begin{array}{c}
B_{\perp} \\
E_{z}
\end{array}\right) .
$$

Таким образом, справедливо

УТВЕРЖДЕНИЕ 2.12. Собственные значения $\gamma_{n}^{2}$ задачи (2.13)-(2.17) расположены симметрично относительно вещественной оси.

Доказательство аналогичного утверждения приведено в [9].

Собственные и присоединенные векторы $A_{1_{n, k}}$ в силу симметрии уравнений $(2.13),(2.14)$ вида (2.36) удовлетворяют обобщенным условиям ортогональности:

$$
\left(\nu d_{2} A_{1_{n, k}}, A_{2_{m, k}}\right)_{L_{2}}=0 \quad \text { при } \quad\left|\gamma_{n}\right|^{2} \neq\left|\gamma_{m}\right|^{2} .
$$

Будем обозначать собственные векторы сопряженной задачи через $A_{n}^{1}$, а собственные векторы исходной - $A_{1_{n}}$. Далее для нас будут представлять интерес собственные векторы сопряженной задачи, отвечающие вещественным $\gamma_{n}$, которые в силу симметрии уравнений (2.13), (2.14) имеют вид

$$
A_{n}^{1}=\nu d_{2} A_{1_{n}}
$$

и являются элементами биортогональной системы к корневым векторам задачи $(2.13)-(2.17)$ :

$$
\left(A_{n}^{1}, A_{1_{m, k}}\right)_{L_{2}}=0, \quad \gamma_{n}^{2} \neq \gamma_{m}^{2} .
$$

Аналогично может быть исследована задача для вектора $A_{2}=\left(D_{\perp}, H_{z}\right)$.

Таким образом, системы собственных и присоединенных векторов спектральной задачи, рассматриваемой как относительно векторов $A_{1}$, так и векторов $A_{2}$, полны в соответствующих функциональных пространствах.

ЗАМЕчАНИЕ 2.6. В частном случае кругового цилиндра и коэффициентов $\varepsilon=\varepsilon(r) I, \mu=I$ легко видеть, что система собственных и присоединенных векторов является базисом со скобками [9] в рассмотренных пространствах [32].

\section{§ 3. Существование решения задачи возбуждения волновода}

Рассмотрим задачу о возбуждении волновода (1.1)-(1.4). Необходимо поставить краевую задачу для уравнений

$$
\begin{aligned}
& a_{1} A_{1}=d_{1} \frac{\partial A_{2}}{\partial z} \\
& a_{2} A_{2}=d_{2} \frac{\partial A_{1}}{\partial z}+J
\end{aligned}
$$

с краевыми условиями и условиями сопряжения (1.3), (1.4).

Требуется дополнить систему уравнений (3.1), (3.2) условиями излучения.

ЗАМЕчАНИЕ 3.1. Для постановки условий излучения используем следующие рассуждения. Вне области, занятой токами, т.е. при $z \leqslant z_{1}$ и $z \geqslant z_{2}$ 
поля $A_{1}, A_{2}$ могут быть приближены суммами вида

$$
\begin{aligned}
& A_{1}=\sum_{n=1}^{M} e^{ \pm i \gamma_{n} z} \sum_{k=1}^{m_{n}} p_{1_{n, k}}(z) A_{1_{n, k}}(x, y), \\
& A_{2}=\sum_{n=1}^{M} e^{ \pm i \gamma_{n} z} \sum_{k=1}^{m_{n}} p_{2_{n, k}}(z) A_{2_{n, k}}(x, y),
\end{aligned}
$$

где $p_{1_{n, k}}(z), p_{2_{n, k}}(z)$ - полиномы от $z$.

Как было установлено в утверждении 2.11, при рассматриваемых условиях эрмитовости матриц $\varepsilon, \mu$ существует конечное число вещественных положительных собственных значений $\gamma_{n}^{2}$, которым отвечают бегущие волны, т. е. не затухающие при $z \rightarrow \pm \infty$ решения. Таким образом, естественно предположить, что решение вне области, занятой токами, представимо в виде суммы конечного числа бегущих волн и стремящегося к нулю при $z \rightarrow \pm \infty$ слагаемого. Отметим, что в случае, если частота поля меньше первой частоты отсечки, то не существует положительных $\gamma_{n}^{2}$ и решение стремится к нулю при $z \rightarrow \pm \infty$.

Итак, можно задать условия излучения следующим образом.

ОПРЕДЕЛЕНИЕ 3.1. Будем говорить, что решение задачи (1.1)-(1.4) удовлетворяет условиям излучения, если при $z<z_{1}$ и $z>z_{2}$ оно представимо в виде

$$
\begin{gathered}
A_{1}=\sum_{n=1}^{N} c_{ \pm n} A_{1_{n}}(x, y) e^{ \pm i \gamma_{n} z}+\bar{A}_{1}, \\
A_{2}=\sum_{n=1}^{N} d_{ \pm n} A_{2_{n}}(x, y) e^{ \pm i \gamma_{n} z}+\bar{A}_{2}, \quad z \leqslant z_{1}, \quad z \geqslant z_{2},
\end{gathered}
$$

где $\bar{A}_{1} \in L_{2}\left(Q_{i}\right), \bar{A}_{2} \in L_{2}\left(Q_{i}\right), Q_{1}=\left\{(x, y) \in \Omega, z \leqslant z_{1}\right\}, Q_{2}=\{(x, y) \in \Omega$, $\left.z \geqslant z_{2}\right\}, c_{ \pm n}, d_{ \pm n}-$ константы.

Задачу для системы (3.1), (3.2) можно свести к задаче для одного уравнения путем введения нового неизвестного вектора $A$, поскольку справедливо

УтвеРЖДЕНИЕ 3.1. Пусть вектор А является решением краевой задачи

$$
a_{2} d_{1}^{-1} a_{1} A=d_{2} \frac{\partial^{2} A}{\partial z^{2}}+J
$$

с граничными условиями и условиями сопряжения

$$
\begin{gathered}
\left.A_{\perp} \boldsymbol{n}\right|_{\partial \Omega}=0,\left.\quad A_{z}\right|_{\partial \Omega}=0, \\
{\left.\left[A_{\perp} \boldsymbol{n}\right]\right|_{S}=0,\left.\quad\left[A_{z}\right]\right|_{S}=0,} \\
{\left.\left[\mu_{33}^{-1} \operatorname{div} A_{\perp}\right]\right|_{S}=0,\left.\quad\left[\varepsilon\left(\operatorname{grad} A_{z}+i k\left(A \times e_{z}\right)\right) \boldsymbol{n}\right]\right|_{S}=0 .}
\end{gathered}
$$

Тогда решения уравнений (3.1), (3.2) определяются по формулам

$$
A_{1}=\frac{\partial A}{\partial z}, \quad A_{2}=d_{1}^{-1} a_{1} A
$$

Задачу (3.5)-(3.7) необходимо дополнить условиями излучения. 
ЗАмечАниЕ 3.2. Отметим, что в области, не занятой токами, поиск решения сводится к решению уравнения

$$
a_{2} d_{1}^{-1} a_{1} A=-\gamma^{2} d_{2} A,
$$

т. е. к задаче (2.13)-(2.17). Для вектора $A$ получаем спектральную задачу такую же, как для вектора $A_{1}$. Поэтому в области, не занятой токами, вектор $A$ имеет тот же вид, что и вектор $A_{1}$. При этом задача (3.9) является уже задачей для линейного операторного пучка и необходимым в дальнейшем условием является обычная, а не кратная полнота векторов $A_{n, k}$.

Введем следующие условия излучения. Пусть существует $N$ положительных вещественных собственных значений $\gamma_{n}^{2}$, которым соответствуют только собственные векторы.

ОПРЕДЕЛЕНИЕ 3.2. Будем говорить, что решение задачи (3.5)-(3.7) удовлетворяет условиям излучения, если при $z<z_{1}$ и $z>z_{2}$ вектор $A$ представим в виде

$$
A=\sum_{n=1}^{N} a_{ \pm n} A_{n}(x, y) e^{ \pm i \gamma_{n} z}+\bar{A}
$$

где $\bar{A} \in L_{2}\left(Q_{i}\right), Q_{i}$ - полуцилиндры, $Q_{1}=\left\{(x, y) \in \Omega, z \in\left(-\infty, z_{1}\right)\right\}, Q_{2}=$ $\left\{(x, y) \in \Omega, z \in\left(z_{2},+\infty\right)\right\}, a_{ \pm n}-$ константа.

ОПРЕДЕЛЕНИЕ 3.3. Обобщенным решением задачи (3.5)-(3.7), (3.10) будем называть вектор $A(x, y, z)$, удовлетворяющий уравнению

$$
a(A, \widetilde{A})+b(A, \widetilde{A})=-\frac{\partial^{2}}{\partial z^{2}} c(A, \widetilde{A})+(J, \widetilde{A})_{L_{2}} \quad \forall \widetilde{A}(x, y) \in W
$$

и условиям (3.10).

Пусть для любого $\widetilde{A}(x, y) \in W$ выполняется условие $\frac{\partial^{2}}{\partial z^{2}} c(A, \widetilde{A}) \in L_{2}^{\text {loc }}$. Предположим также, что при почти всех $z$ выполняется условие $A \in W$.

При сделанном предположении о том, что частота электромагнитного поля не совпадает с частотой отсечки, множество собственных значений задачи (3.9) состоит из нулевого собственного значения, которому соответствуют собственные векторы вида $(\operatorname{rot} \phi, i k \phi)^{T}$ для любого $\phi \in \dot{H}^{1}$, и ненулевых собственных значений, которым соответствуют корневые векторы, принадлежащие пространству $V$.

ЗАмечАнИЕ 3.3. Вектор $J$ не удовлетворяет условию (2.22), которому удовлетворяют корневые векторы, отвечающие ненулевым собственным значениям.

Будем использовать следующий метод построения решения $A \in W$. Запишем уравнение (3.11) в виде

$$
a(A, \widetilde{A})+b(A, \widetilde{A})=\frac{\partial^{2}}{\partial z^{2}} c(A, \widetilde{A})+c\left(d_{2}^{-1} J, \widetilde{A}\right) \quad \forall \widetilde{A} \in W .
$$

Представим вектор $J$ в виде суммы двух слагаемых:

$$
J=J_{1}+J_{2}
$$


где $J_{1}$ удовлетворяет условию $(2.22)$, причем $d_{2}^{-1} J_{2}$ принадлежит ядру спектральной задачи (2.21), рассматриваемой в пространстве $W$. Возможность подобного представления будет доказана ниже. Разобьем исследование задачи (3.12), (3.10) на следующие задачи.

ЗАДАчА 3.1. Найти вектор $A \in W, \frac{\partial^{2}}{\partial z^{2}} A \in L_{2}^{\text {loc }}$, удовлетворяющий уравнению

$$
a(A, \widetilde{A})+b(A, \widetilde{A})=\frac{\partial^{2}}{\partial z^{2}} c(A, \widetilde{A})+c\left(d_{2}^{-1} J_{2}, \widetilde{A}\right) \quad \forall \widetilde{A} \in W
$$

с условиями

$$
\frac{\partial A}{\partial z}=0, \quad z \leqslant z_{1}, \quad \frac{\partial A}{\partial z}=0, \quad z \geqslant z_{2} .
$$

Данные условия соответствуют тому, что при $z \leqslant z_{1}$ и $z \geqslant z_{2}$ поле $\frac{\partial A}{\partial z}=A_{1}$ обращается в нуль, так как ток $J_{2}$ не принадлежит пространству $V$ и возбуждает поле в ближней зоне. Поскольку требуется найти поле, а не потенциал, то можно ограничиться поиском $\frac{\partial A}{\partial z}$. При этом, как легко видеть,

$$
A_{1}=\frac{\partial A}{\partial z}, \quad A_{2}=0 .
$$

Ток $J_{1}$ представим в виде суммы:

$$
J_{1}=J_{1}^{N}+\bar{J}_{1}
$$

Ток $J_{1}^{N}$ представляет собой проекцию вектора $J_{1}$ на конечномерное пространство собственных векторов (которые будем обозначать $A_{n}$ ), отвечающих бегущим волнам. Собственные векторы сопряженной задачи обозначим $A^{n}=$ $\nu d_{2}^{-1} A_{n}$.

ЗАДАчА 3.2. Для вектора $J_{1}^{N}=\sum_{n=1}^{N}\left(J_{1}, A^{n}\right)_{L_{2}} A_{n}$ рассмотрим следующую задачу:

$$
a(A, \widetilde{A})+b(A, \widetilde{A})=\frac{\partial^{2}}{\partial z^{2}} c(A, \widetilde{A})+c\left(J_{1}^{N}, \widetilde{A}\right) \quad \forall \widetilde{A} \in W
$$

с условиями излучения при $z \leqslant z_{1}$ и $z \geqslant z_{2}$ :

$$
A=\sum_{n=1}^{N} a_{ \pm n} A_{n} e^{ \pm i \gamma_{n} z} .
$$

Решение данной задачи сводится к решению конечного числа обыкновенных дифференциальных уравнений.

ЗАДАчА 3.3. Ток $\bar{J}_{1}$ удовлетворяет условиям ортогональности

$$
c\left(\bar{J}_{1}, A^{n}\right)=0, \quad n=1, \ldots, N .
$$

При этих условиях, налагаемых на правую часть уравнения (3.11), существует решение уравнения

$$
a(A, \widetilde{A})+b(A, \widetilde{A})=\frac{\partial^{2}}{\partial z^{2}} c(A, \widetilde{A})+c\left(\bar{J}_{1}, \widetilde{A}\right) \quad \forall \widetilde{A} \in W
$$

удовлетворяющее условиям $A \in L_{2}(W ;(-\infty,+\infty))$. 
Сумма решений трех задач (3.13)-(3.15) удовлетворяет уравнению (3.11) и условиям излучения (3.10). Приступим к строгому построению её решения.

Напомним, что частота $k$ электромагнитного поля не совпадает с частотой отсечки. Докажем следующую теорему о разложении произвольного векторного поля.

TeOpema 3.1. Пусть $A \in L_{2}(\Omega)$. Найдутся $\phi \in \dot{H}^{1}$ u $F \in H(\operatorname{div}) \oplus H^{1}$ maкue, чmo

$$
A=\left(\begin{array}{cc}
\mu^{-1} & 0 \\
0 & \varepsilon_{33}
\end{array}\right)\left(\begin{array}{c}
\operatorname{rot} \phi \\
i k \phi
\end{array}\right)+\left(\begin{array}{cc}
-i k \mathcal{J} & \operatorname{grad} \\
-\operatorname{div} & 0
\end{array}\right)\left(\begin{array}{c}
F_{\perp} \\
F_{z}
\end{array}\right),
$$

или $A=d_{2}(\operatorname{rot} \phi, i k \phi)^{T}+a_{2} F$.

ДоКАЗАТЕЛЬСТВо. Пусть $\phi \in \dot{H}^{1}$ является обобщенным решением задачи

$$
\left(\mu^{-1} \operatorname{rot} \phi, \operatorname{rot} \tilde{\phi}\right)_{L_{2}}-k^{2}\left(\varepsilon_{33} \phi, \tilde{\phi}\right)_{L_{2}}=\left(A_{\perp}, \operatorname{rot} \tilde{\phi}\right)_{L_{2}}+i k\left(A_{z}, \tilde{\phi}\right)_{L_{2}} \quad \forall \tilde{\phi} \in \dot{H}^{1} .
$$

Поскольку частота электромагнитного поля не совпадает с частотой отсечки, данная задача имеет единственное решение. Запишем уравнение (3.16) в виде

$$
\left(\mu^{-1} \operatorname{rot} \phi-A_{\perp}, \operatorname{rot} \tilde{\phi}\right)_{L_{2}}+i k\left(i k \varepsilon_{33} \phi-A_{z}, \tilde{\phi}\right)_{L_{2}}=0 \quad \forall \tilde{\phi} \in \dot{H}^{1} .
$$

Обозначим $\bar{A}=\left(\bar{A}_{\perp}, \bar{A}_{z}\right)^{T}$, где

$$
\bar{A}_{\perp}=\mu^{-1} \operatorname{rot} \phi-A_{\perp}, \quad \bar{A}_{z}=i k \varepsilon_{33} \phi-A_{z} .
$$

Вектор $\bar{A}$ удовлетворяет уравнению

$$
\left(\bar{A}_{\perp}, \operatorname{rot} \tilde{\phi}\right)_{L_{2}}+i k\left(\bar{A}_{z}, \tilde{\phi}\right)_{L_{2}}=0 \quad \forall \tilde{\phi} \in \dot{H}^{1} .
$$

Представим вектор $\mu \bar{A}_{\perp}$ в виде

$$
\mu \bar{A}_{\perp}=\mu \operatorname{grad} \psi+\operatorname{rot} \chi, \quad \psi \in H^{1}, \quad \chi \in \dot{H}^{1} .
$$

Подставляя вектор $A_{\perp}$ в уравнение (3.17) и пользуясь определением вектора $\bar{A}_{\perp}$, получим

$$
(\operatorname{grad} \psi, \operatorname{rot} \tilde{\phi})_{L_{2}}+\left(\mu^{-1} \operatorname{rot} \chi, \operatorname{rot} \tilde{\phi}\right)_{L_{2}}+i k\left(\bar{A}_{z}, \tilde{\phi}\right)_{L_{2}}=0 \quad \forall \tilde{\phi} \in \dot{H}^{1},
$$

или $\left(\bar{A}_{z}, \tilde{\phi}\right)_{L_{2}}=\frac{i}{k}\left(\mu^{-1} \operatorname{rot} \chi, \operatorname{rot} \tilde{\phi}\right)_{L_{2}}$.

Определение пространства $H(\mathrm{rot})$ аналогично определению $H(\operatorname{div})$ [3]:

$$
H(\operatorname{rot})=\left\{A \in L_{2}(\Omega), \operatorname{rot} H \in L_{2}(\Omega)\right\} .
$$

Отсюда получаем, что $\mu^{-1} \operatorname{rot} \chi \in H(\operatorname{rot})$ и справедливо равенство

$$
\bar{A}_{z}=\frac{i}{k} \operatorname{rot} \mu^{-1} \operatorname{rot} \chi
$$

Запишем равенство (3.18) в виде

$$
\bar{A}_{z}=\frac{i}{k} \operatorname{div}\left(\mathcal{J} \mu^{-1} \mathcal{J} \operatorname{grad} \chi\right),
$$


где $\mathcal{J} \mu^{-1} \mathcal{J} \operatorname{grad} \chi \in H(\operatorname{div})$. Поскольку $\bar{A}_{\perp}=\operatorname{grad} \psi+\mu^{-1} \mathcal{J} \operatorname{grad} \chi$, то получим

$$
\left(\begin{array}{c}
A_{\perp} \\
A_{z}
\end{array}\right)=\left(\begin{array}{cc}
\mu^{-1} & 0 \\
0 & \varepsilon_{33}
\end{array}\right)\left(\begin{array}{c}
\operatorname{rot} \phi \\
i k \phi
\end{array}\right)+\left(\begin{array}{cc}
-i k \mathcal{J} & \operatorname{grad} \\
-\operatorname{div} & 0
\end{array}\right)\left(\begin{array}{c}
-\frac{i}{k} \mathcal{J} \mu^{-1} \mathcal{J} \operatorname{grad} \chi \\
\psi
\end{array}\right) .
$$

Обозначим

$$
\begin{gathered}
F_{\perp}=-\frac{i}{k} \mathcal{J} \mu^{-1} \mathcal{J} \operatorname{grad} \chi, \\
F_{z}=\psi,
\end{gathered}
$$

причем в силу построения имеем $\left(F_{\perp}, F_{z}\right) \in H(\operatorname{div}) \oplus H^{1}$. Таким образом,

$$
\begin{aligned}
\left(\begin{array}{c}
A_{\perp} \\
A_{z}
\end{array}\right) & =\left(\begin{array}{cc}
\mu^{-1} & 0 \\
0 & \varepsilon_{33}
\end{array}\right)\left(\begin{array}{c}
\operatorname{rot} \phi \\
i k \phi
\end{array}\right)+\left(\begin{array}{cc}
-i k \mathcal{J} & \operatorname{grad} \\
-\operatorname{div} & 0
\end{array}\right)\left(\begin{array}{c}
F_{\perp} \\
F_{z}
\end{array}\right) \\
& =d_{2}(\operatorname{rot} \phi, i k \phi)^{T}+a_{2} F .
\end{aligned}
$$

Теорема доказана.

Вернемся к рассмотрению уравнения

$$
a(A, \widetilde{A})+b(A, \widetilde{A})=\frac{\partial^{2}}{\partial z^{2}} c(A, \widetilde{A})+c\left(d_{2}^{-1} J, \widetilde{A}\right) .
$$

Представим вектор $J$ в виде

$$
J=d_{2}(\operatorname{rot} \phi, i k \phi)^{T}+a_{2} F .
$$

Тогда $d_{2}^{-1} J=(\operatorname{rot} \phi, i k \phi)^{T}+d_{2}^{-1} a_{2} F$, причем $d_{2}^{-1} a_{2} F$ удовлетворяет уравнению $(2.22)$. По построению $(\operatorname{rot} \phi, i k \phi) \in W$. Действительно, поскольку $\left.\phi\right|_{\partial \Omega}=0$, то $\left.\operatorname{rot} \phi \boldsymbol{n}\right|_{\partial \Omega}=0$ и $\operatorname{rot} \phi \in H(\operatorname{div})$. Вектор $d_{2}^{-1} a_{2} F$ удовлетворяет условиям $\left.d_{2}^{-1} a_{2} F \boldsymbol{n}\right|_{\partial \Omega}=0,\left.\quad\left(d_{2}^{-1} a_{2} F\right)_{z}\right|_{\partial \Omega}=0$ в силу равенства (3.22). Обозначим

$$
J_{1}=a_{2} F, \quad J_{2}=d_{2}(\operatorname{rot} \phi, i k \phi) .
$$

Вернемся к задаче 3.1: найти вектор $A \in W$, удовлетворяющий задаче

$$
\begin{gathered}
a(A, \widetilde{A})+b(A, \widetilde{A})=\frac{\partial^{2}}{\partial z^{2}} c(A, \widetilde{A})+c\left(d_{2}^{-1} J_{2}, \widetilde{A}\right) \quad \forall \widetilde{A} \in W, \\
\left.\frac{\partial A}{\partial z}\right|_{z \leqslant z_{1}}=0, \\
\left.\frac{\partial A}{\partial z}\right|_{z \geqslant z_{2}}=0 .
\end{gathered}
$$

Ищем $A$ в виде $A=(\operatorname{rot} \psi, i k \psi)$. По существу нам требуется найти $\frac{\partial A}{\partial z}$, чем мы и ограничимся. Имеем

$$
\frac{\partial^{2}}{\partial z^{2}} c(A, \widetilde{A})+c\left(d_{2}^{-1} J_{2}, \widetilde{A}\right)=0 \quad \forall \widetilde{A} \in W .
$$

Таким образом, получим

$$
\frac{\partial A}{\partial z}=\int_{z_{1}}^{z} J_{2} d z^{\prime}=\int_{z_{1}}^{z}(\operatorname{rot} \phi, i k \phi) d z^{\prime}
$$


Покажем, что выполняется условие $\left.\frac{\partial A}{\partial z}\right|_{z \geqslant z_{2}}=0$ и задача не является переопределенной. Действительно, $\frac{\partial A}{\partial z}=(\operatorname{rot} \psi, i k \psi)$, где $\psi=\int_{z_{1}}^{z} \phi d z^{\prime}$.

При любом $\tilde{\phi}(x, y) \in \dot{H}^{1}$ справедливо равенство

$$
\begin{aligned}
& \left(\mu^{-1} \operatorname{rot} \psi, \operatorname{rot} \tilde{\phi}\right)-k^{2}\left(\varepsilon_{33} \psi, \tilde{\phi}\right)=\int_{z_{1}}^{z}\left(\left(\mu^{-1} \operatorname{rot} \phi, \operatorname{rot} \tilde{\phi}\right)_{L_{2}}-k^{2}\left(\varepsilon_{33} \phi, \tilde{\phi}\right)_{L_{2}}\right) d z^{\prime} \\
& \quad=\int_{z_{1}}^{z}\left(\left(J_{\perp}, \operatorname{rot} \tilde{\phi}\right)_{L_{2}}+i k\left(J_{z}, \tilde{\phi}\right)_{L_{2}}\right) d z^{\prime} \\
& \quad=-\int_{z_{1}}^{z}\left(\left(\operatorname{div} J_{\perp}, \tilde{\phi}\right)_{L_{2}}-i \omega\left(j_{z}, \tilde{\phi}\right)\right) d z^{\prime}=\int_{z_{1}}^{z}\left(\frac{\partial j_{z}}{\partial z}, \tilde{\phi}\right)_{L_{2}} d z^{\prime}=0
\end{aligned}
$$

в силу того, что $j_{z}=0$ при $z \geqslant z_{2}$ и $\operatorname{div} j_{\perp}-i \omega \rho=-\frac{\partial j_{z}}{\partial z}$. Таким образом, получим

$$
\left(\mu^{-1} \operatorname{rot} \psi, \operatorname{rot} \tilde{\phi}\right)_{L_{2}}-k^{2}\left(\varepsilon_{33} \psi, \tilde{\phi}\right)_{L_{2}}=0 \quad \forall \tilde{\phi} \in \dot{H}^{1} .
$$

В силу того, что $\psi \in \dot{H}^{1}(\Omega)$ и $k^{2}$ не совпадает с частотой отсечки, имеем $\psi=0$ для любого $z \geqslant z_{2}$, отсюда $\frac{\partial A}{\partial z}=0$ как предел справа для любого $z \geqslant z_{2}$. Следовательно, найдено решение задачи (3.23)-(3.25).

Рассмотрим задачу относительно вектора $J_{1}$. Система корневых векторов задачи

$$
a(A, \widetilde{A})+b(A, \widetilde{A})=-\gamma^{2} c(A, \widetilde{A}) \quad \forall \widetilde{A} \in V
$$

полна в пространстве $V$. Представим пространство $V$ в виде суммы пространства $V_{N}$ - линейной оболочки собственных векторов, отвечающих собственным значениям $\gamma_{n}^{2}>0$, и пространства $V_{1}$ - замыкания линейной оболочки остальных корневых векторов: $V=V_{N} \oplus V_{1}$. Представим вектор $J_{1}$ в виде $J_{1}=J_{1}^{N}+\bar{J}_{1}$, где $J_{1}^{N}=\sum_{n=1}^{N}\left(J_{1}, A^{n}\right)_{L_{2}} A_{n}$. Кроме того,

$$
\left(J_{1}, A^{n}\right)_{L_{2}}=\left(J, A^{n}\right)_{L_{2}}-\left(d_{2}(\operatorname{rot} \phi, i k \phi)^{T}, A^{n}\right)_{L_{2}}=\left(J, A^{n}\right)_{L_{2}},
$$

поскольку $\left(d_{2}(\operatorname{rot} \phi, i k \phi)^{T}\right)=0$. Таким образом, коэффициенты $J_{n}=\left(J_{1}, A^{n}\right)_{L_{2}}$ вычисляются через вектор $J$.

Рассмотрим задачу 3.2. Будем искать вектор $A \in V_{N}$ вида

$$
A=\sum_{n=1}^{N} Z_{n}(z) A_{n}(x, y),
$$

удовлетворяющий уравнению

$$
a(A, \widetilde{A})+b(A, \widetilde{A})=\frac{\partial^{2}}{\partial z^{2}} c(A, \widetilde{A})+c\left(d_{2} J_{1}^{N}, \widetilde{A}\right) \quad \forall A \in W
$$

и условиям излучения

$$
\begin{aligned}
& A=\sum_{n=1}^{N} a_{+n} A_{n}(x, y) e^{i \gamma_{n} z}, \quad z \geqslant z_{2}, \\
& A=\sum_{n=1}^{N} a_{-n} A_{n}(x, y) e^{-i \gamma_{n} z}, \quad z \leqslant z_{1} .
\end{aligned}
$$


Учитывая, что векторы $A_{n}(x, y)$ удовлетворяют условиям $\left(A_{n}, A^{m}\right)=0$ при $\gamma_{n} \neq \gamma_{m}$, где $A^{m}=\nu d_{2} A_{n}$, получим следующие краевые условия:

$$
\begin{array}{ll}
\frac{\partial A}{\partial z}=\sum_{n=1}^{N} i \gamma_{n}\left(A, A^{n}\right)_{L_{2}} A_{n}, & z \geqslant z_{2}, \\
\frac{\partial A}{\partial z}=-\sum_{n=1}^{N} i \gamma_{n}\left(A, A^{n}\right)_{L_{2}} A_{n}, & z \leqslant z_{1} .
\end{array}
$$

Отсюда для функций $Z_{n}$ получим

$$
\begin{gathered}
Z_{n}^{\prime \prime}+\gamma_{n}^{2} Z_{n}=J_{n}(z)=\left(J, A^{n}\right)_{L_{2}}, \\
\left.\left(Z_{n}^{\prime}-i \gamma_{n} Z_{n}\right)\right|_{z=z_{2}}=0, \\
\left.\left(Z_{n}^{\prime}+i \gamma_{n} Z_{n}\right)\right|_{z=z_{1}}=0 .
\end{gathered}
$$

В результате определяется $Z_{n}(z)$. Таким образом, может быть построено решение задачи (3.31)-(3.33), которое имеет вид

$$
Z_{n}(z)=\frac{i}{2 \gamma_{n}} \int_{-\infty}^{+\infty} e^{i \gamma_{n}\left|z-z^{\prime}\right|} J_{n}\left(z^{\prime}\right) d z^{\prime}
$$

Рассмотрим задачу 3.3 в случае, когда правой частью является $\bar{J}_{1}$. В силу того, что $\left(\bar{J}_{1}, A^{n}\right)=0, n=1, \ldots, N$, вектор $\bar{J}_{1}$ принадлежит $V_{1}$. Будем рассматривать задачу

$$
a(A, \widetilde{A})+b(A, \widetilde{A})=\frac{\partial^{2}}{\partial z^{2}} c(A, \widetilde{A})+c\left(J_{1}, \widetilde{A}\right) \quad \forall \widetilde{A} \in W .
$$

При этом будем искать $A \in L_{2}(W ;(-\infty,+\infty))$.

Справедливо следующее

УТВЕРЖДЕНИЕ 3.2. Пусть уравнение (3.34) выполнено для любого $\widetilde{A} \in V_{1}$. Тогда оно выполнено для любого $\widetilde{A} \in W$.

Действительно, $V_{1}$ является замыканием линейной оболочки корневых векторов задачи $(2.21)$, удовлетворяющих условиям $\left(J_{1}, A^{n}\right)_{L_{2}}=0, n=1, \ldots, N$. При этом если $A$ является решением задачи и элементом пространства $V_{1}$, то

$$
c(A, \widetilde{A})=0, \quad \frac{\partial^{2}}{\partial z^{2}} c(A, \widetilde{A})=0 \quad \forall \widetilde{A} \in V_{1}^{\perp},
$$

где $V_{1}^{\perp}$ - ортогональное дополнение пространства $V_{1}$ до пространства $W$ относительно скалярного произведения, порождаемого $c(A, \widetilde{A})$. Поскольку система векторов $A_{n}$ полна в пространстве $V_{1}$ и

$$
a\left(A_{n}, \widetilde{A}\right)+b\left(A_{n}, \widetilde{A}\right)=-\gamma_{n}^{2} c\left(A_{n}, \widetilde{A}\right)=0 \quad \forall \widetilde{A} \in V_{1}^{\perp},
$$

то

$$
a(A, \widetilde{A})+b(A, \widetilde{A})=0 \quad \forall \widetilde{A} \in V_{1}^{\perp} .
$$

Вектор $\bar{J}_{1}$ по построению удовлетворяет условию $c\left(d_{2}^{-1} \bar{J}_{1}, \widetilde{A}\right)=0$ для любого $\widetilde{A} \in V_{1}^{\perp}$. Таким образом, решение задачи, рассматриваемой в пространстве $V_{1}$, 
удовлетворяет задаче в пространстве $W$, т. е. именно в том пространстве, в котором рассматривается исходная задача. Будем рассматривать далее уравнение в пространстве $V_{1}$.

Предположим, что решение задачи существует. Для преобразования Фурье

$$
\widehat{A}=\frac{1}{2 \pi} \int_{-\infty}^{+\infty} e^{i \gamma z} A(x, y, z) d z
$$

решения $A$ получим задачу

$$
(I+C) \widehat{A}=-\gamma^{2} H \widehat{A}+\widehat{F},
$$

где $F$ определяется равенством $a(F, \widetilde{A})=c\left(d_{2} \bar{J}_{1}^{-1}, \widetilde{A}\right)$ для любого $\widetilde{A} \in V_{1}$. Вектор $\widehat{F}$ представляет собой преобразование Фурье вектора $F$. Вектор $J_{1}$ принадлежит $\left(L_{2}\right)^{3}(\Omega \times(-\infty,+\infty))$, поскольку $J_{1}$ отлично от нуля лишь на отрезке $\left[z_{1}, z_{2}\right]$. Отсюда следует, что $J_{1}(x, y, z) \in L_{2}(-\infty,+\infty)$ при почти всех $(x, y)$, поэтому можно определить преобразование Фурье вектора $F$ при почти всех $(x, y)$.

Покажем, что справедлива

Лемма 3.1. Существует решение задачи (3.34).

Обратное преобразование Фурье решения задачи (3.35) представляет собой решение исходной задачи (3.34). Определим вектор $A$ следующим образом:

$$
A=\int_{-\infty}^{+\infty} e^{i \gamma z}\left(I+C+\gamma^{2} H\right)^{-1} \widehat{F}(x, y, \gamma) d \gamma
$$

Поскольку все собственные значения $\gamma^{2}$ лежат внутри некоторого сектора, вне которого резольвента $\left(I+C+\gamma^{2} H\right)^{-1}$ равномерно ограничена по $\gamma$, то существует $\gamma_{\max }$ такое, что при $\gamma<-\gamma_{\max }$ и $\gamma>\gamma_{\max }$ резольвента ограничена некоторой константой и выполнено

$$
\begin{gathered}
\left\|H\left(I+C+\gamma^{2} H\right)^{-1}\right\|_{W} \leqslant C_{1} \frac{1}{\gamma^{2}}, \quad \gamma \in\left(-\infty,-\gamma_{\max }\right) \cup\left(\gamma_{\max },+\infty\right), \\
\left\|\left(I+C+\gamma^{2} H\right)^{-1} \widehat{F}\right\|_{W} \leqslant M, \quad \gamma \in\left[-\gamma_{\max }, \gamma_{\max }\right] .
\end{gathered}
$$

Допустим, существует точка на вещественной оси, в которой резольвента неограничена. Тогда эта точка является собственным значением пучка $I+C+\gamma^{2} H$ в силу компактности пучка. Однако данный пучок не имеет вещественных собственных значений. Поскольку резольвента $\left(I+C+\gamma^{2} H\right)^{-1}$ ограничена в каждой точке $\gamma$ отрезка $\left[-\gamma_{\max }, \gamma_{\max }\right]$ и является аналитической функцией, то найдется такая константа $M$, что

$$
\left\|\left(I+C+\gamma^{2} H\right)^{-1}\right\|_{W} \leqslant M, \quad \gamma \in\left[-\gamma_{\max }, \gamma_{\max }\right] .
$$

Следовательно, $\left\|\left(I+C+\gamma^{2} H\right)^{-1} \widehat{F}\right\|_{W} \in L_{2}(-\infty,+\infty)$. Отсюда получим

$$
\left(I+C+\gamma^{2} H\right)^{-1} \widehat{F} \in\left(L_{2}\right)^{3}(Q), \quad Q=\Omega \times(-\infty,+\infty) .
$$

Таким образом, при почти всех $\gamma$ определен вектор $\left(I+C+\gamma^{2} H\right)^{-1} \widehat{F} \in$ $L_{2}(-\infty,+\infty)$, поэтому имеет смысл определение вектора $A$, причем при почти всех $(x, y)$ вектор $A(x, y) \in L_{2}(-\infty,+\infty)$, т. е. $\int_{-\infty}^{+\infty}|A(x, y, z)|^{2} d z<\infty$. 
Оценим норму оператора $H\left(I+C+\gamma^{2} H\right)^{-1}$ при $\gamma \in\left(-\infty,-\gamma_{\max }\right) \cup\left(\gamma_{\max },+\infty\right)$. Справедливо равенство

$$
H\left(I+C+\gamma^{2} H\right)^{-1}=\frac{1}{\gamma^{2}} I-\frac{1}{\gamma^{2}}(I+C)\left(I+C+\gamma^{2} H\right)^{-1} .
$$

Отсюда следует, что

$$
\left\|H\left(I+C+\gamma^{2} H\right)^{-1}\right\|_{W}=\left\|\frac{1}{\gamma^{2}} I-\frac{1}{\gamma^{2}}(I+C)\left(I+C+\gamma^{2} H\right)^{-1}\right\|_{W} .
$$

В силу того, что $\left\|\left(I+C+\gamma^{2} H\right)^{-1}\right\|_{W} \leqslant M$ при $\gamma \leqslant-\gamma_{\max }$ и $\gamma \geqslant \gamma_{\max }$, справедлива оценка

$$
\left\|H\left(I+C+\gamma^{2} H\right)^{-1}\right\|_{W} \leqslant \frac{M_{1}}{\gamma^{2}}, \quad \gamma \in\left(-\infty,-\gamma_{\max }\right) \cup\left(\gamma_{\max },+\infty\right) .
$$

Таким образом, $\frac{\partial^{2}}{\partial z^{2}} H A \in L_{2}(-\infty,+\infty)$ при почти всех $(x, y)$. Действительно, это справедливо в силу неравенства

$$
\left\|H\left(I+C+\gamma^{2} H\right)^{-1} \widehat{F}(x, y, \gamma)\right\|_{W} \leqslant \frac{M_{2}}{\gamma^{2}}
$$

при $\gamma \in\left(-\infty,-\gamma_{\max }\right) \cup\left(\gamma_{\max },+\infty\right)$. Отсюда имеем, что норма $\left\|\gamma^{2} H A\right\|_{W}$ как функция от $\gamma$ является элементом пространства $L_{2}(-\infty,+\infty)$ и, следовательно, в силу свойств преобразования Фурье $\frac{\partial^{2}}{\partial z^{2}} H A \in L_{2}(Q)$. Покажем, что $A \in V_{1}$ является решением задачи

$$
((I+C) A, \widetilde{A})_{V}=\frac{\partial^{2}}{\partial z^{2}}(H A, \widetilde{A})_{V}+\left(H J_{1}, \widetilde{A}\right)_{V} \quad \forall \widetilde{A} \in V_{1} .
$$

Действительно,

$$
\begin{aligned}
\frac{\partial^{2}}{\partial z^{2}} H A & =-\int_{-\infty}^{+\infty} \gamma^{2} e^{i \gamma z} H\left(I+C+\gamma^{2} H\right)^{-1} \widehat{F} d \gamma \\
& =\int_{-\infty}^{+\infty} e^{i \gamma z} \widehat{F}(x, y, \gamma) d \gamma-\int_{-\infty}^{+\infty} e^{i \gamma z}(I+C)\left(I+C+\gamma^{2} H\right)^{-1} \widehat{F} d \gamma \\
& =F(x, y, z)-(I+C) A(x, y, z) .
\end{aligned}
$$

Отсюда следует, что выполняется (3.34), т. е. доказано существование решения задачи

$$
a(A, \widetilde{A})+b(A, \widetilde{A})=\frac{\partial^{2}}{\partial z^{2}} c(A, \widetilde{A})+\left(\bar{J}_{1}, \widetilde{A}\right) \quad \forall \widetilde{A} \in W,
$$

где $A \in L_{2}(V ;(-\infty,+\infty))$.

ЗАмечАнИЕ 3.4. Из уравнения (3.39) следует, что существует $\frac{\partial A}{\partial z} \in L_{2}(Q)$. Доказательство осуществляется стандартным методом.

Векторы $A_{1}$ и $A_{2}$ удовлетворяют уравнениям (3.1), (3.2) и являются элементами пространства $L_{2}(Q)$. 


\section{§4. Моды полого волновода}

В частном случае $\varepsilon=I, \mu=I$ присоединенные векторы отсутствуют и система собственных векторов образует ортонормированный базис пространства $V$. При этом ядро задачи, т. е. множество векторов вида $(\operatorname{rot} \psi, i k \psi), \psi \in \dot{H}^{1}$, pacсматриваемой в пространстве $W$, не будет ортогонально собственным векторам.

УтВЕРЖДЕНИЕ 4.1. Пусть $\psi_{n}(x, y)$ - собственные функиии задачи

$$
-\Delta_{\perp} \psi=\lambda \psi,\left.\quad \frac{\partial \psi}{\partial \boldsymbol{n}}\right|_{\Omega}=0
$$

Тогда векторы $A_{n}=\operatorname{grad}_{\perp} \psi_{n}$ являются решениями задачи (2.13)-(2.17).

УТВеРЖДЕНИЕ 4.2. Пусть $\chi_{n}-$ собственные функиии задачи

$$
-\Delta_{\perp} \chi=\lambda \chi,\left.\quad \chi\right|_{\partial \Omega}=0 .
$$

Тогда $A_{n}=-i k \operatorname{rot} \chi_{n}+\lambda_{n} \chi_{n} e_{z}-$ решения задачи (2.13), (2.14).

Доказательства утверждений 4.1, 4.2 осуществляются непосредственной проверкой. Легко видеть, что система векторов $A_{n}$ ортогональна в $L_{2}$.

\section{§ 5. Пример комплексных собственных значений}

Задача поиска нормальных волн регулярных волноводов сводится к спектральной задаче для несамосопряженного оператора даже в случае отсутствия потерь в среде. Собственные значения могут быть комплексными, при этом в случае кратных собственных значений, помимо собственных векторов, могут существовать присоединенные векторы. Кроме того, возможны участки дисперсионных кривых, для которых $\frac{d \gamma}{d k}<0$. (С физической точки зрения это означает, что фазовая и групповая скорости волны направлены в противоположные стороны.) Примеры подобных эффектов приведены в работах [33], [34].

В настоящем параграфе показано, что подобные эффекты возникают для волновода квадратного сечения с анизотропным заполнением даже в случае постоянных коэффициентов. Решение этой задачи выписывается в явном виде.

Считаем, что сечение $\Omega$ цилиндра - единичный квадрат:

$$
\Omega=\{0 \leqslant x \leqslant 1,0 \leqslant y \leqslant 1\} .
$$

Коэффициенты системы уравнений Максвелла имеют следующий вид. Матрица диэлектрической проницаемости - диагональная: $\varepsilon_{i j}=\varepsilon_{i} \delta_{i j}$, матрица магнитной проницаемости - единичная: $\mu=I$.

Будем искать решения задачи (2.13)-(2.17) в виде

$$
\begin{aligned}
H_{x} & =-i H_{x} \sin (\pi n x) \cos (\pi m y), \\
H_{y} & =-i H_{y} \cos (\pi n x) \sin (\pi m y), \\
E_{z} & =E_{z} \sin (\pi n x) \sin (\pi m y),
\end{aligned}
$$

где $H_{x}, H_{y}, E_{z}$ - константы, $n, m=1,2, \ldots$. 
Введем следующие обозначения:

$$
\begin{aligned}
& a_{11}=\pi^{2} n^{2}+\pi^{2} m^{2} \frac{\varepsilon_{2}}{\varepsilon_{3}}-k^{2} \varepsilon_{2}, \quad a_{12}=\pi^{2} n m\left(1-\frac{\varepsilon_{1}}{\varepsilon_{3}}\right), \\
& a_{21}=\pi^{2} n m\left(1-\frac{\varepsilon_{2}}{\varepsilon_{3}}\right), \quad a_{22}=\pi^{2} m^{2}+\pi^{2} n^{2} \frac{\varepsilon_{1}}{\varepsilon_{3}}-k^{2} \varepsilon_{1} .
\end{aligned}
$$

Собственные значения $\gamma^{2}$ вычисляются явно:

$$
\gamma^{2}=\frac{-\left(a_{11}+a_{22}\right) \pm \sqrt{\left(a_{11}-a_{22}\right)^{2}+4 a_{12} a_{21}}}{2} .
$$

Таким образом, собственные значения являются комплексными при условии

$$
\left(a_{11}-a_{22}\right)^{2}+4 a_{12} a_{21}<0 .
$$

Следовательно, необходимым условием существования комплексных собственных значений является условие $\varepsilon_{1}<\varepsilon_{3}<\varepsilon_{2}$ или $\varepsilon_{2}<\varepsilon_{3}<\varepsilon_{1}$. Выбирая $n=1$, $m=1$, легко увидим, что, помимо вещественных, возникают комплексные собственные значения.

Приведем примеры дисперсионных кривых, т. е. зависимостей собственных значений $\gamma^{2}$ от $k^{2}$ при $n=1, m=1$ для $\varepsilon_{1}=1.5, \varepsilon_{2}=8$. Величина $\varepsilon_{3}$ изменяется от 1 до 9.8. На рис. 2 показана типичная дисперсионная кривая $\operatorname{Re} \gamma^{2}\left(k^{2}\right)$. В точке пересечения двух вещественных ветвей дисперсионной кривой происходит переход значений функции $\gamma^{2}\left(k^{2}\right)$ от вещественных к комплексным.

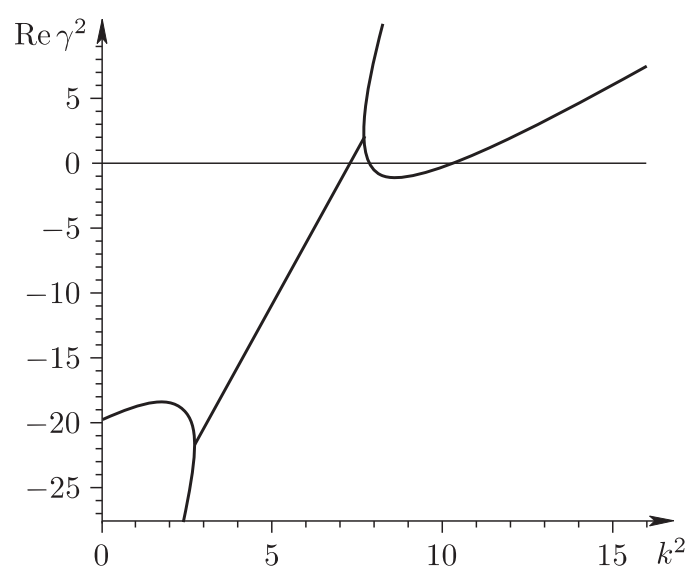

Рис. 2

При этом $\frac{d \gamma^{2}}{d k^{2}}=\infty$, т. е. эта точка является точкой касания двух ветвей дисперсионной кривой. Существуют два таких значения $k^{2}$, которые находятся из условия $\left(a_{11}-a_{22}\right)^{2}+4 a_{12} a_{21}=0$. В точках $k^{2}$, в которых возникает кратное значение функции $\gamma^{2}\left(k^{2}\right)$, существует присоединенный вектор задачи (2.13)-(2.17). Участок линейности вещественной части дисперсионной кривой отвечает комплексным значениям функции $\gamma^{2}\left(k^{2}\right)$.

Отметим, что нижняя ветвь дисперсионной кривой

$$
\gamma^{2}=\frac{-\left(a_{11}+a_{22}\right)-\sqrt{\left(a_{11}-a_{22}\right)^{2}+4 a_{12} a_{21}}}{2}
$$




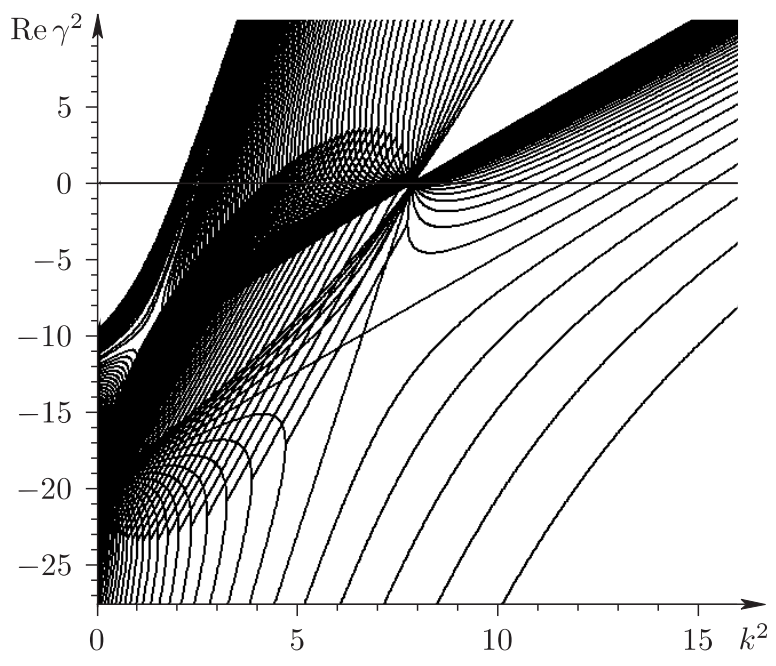

Рис. 3

на рис. 2 имеет участки, на которых $\frac{d \gamma^{2}}{d k^{2}}<0$.

Эволюция дисперсионных кривых в интервале от $\varepsilon_{3}=1$ до $\varepsilon_{3}=9.8$ показана на рис. 3.

\section{§6. Вещественные собственные значения}

Рассмотрим вопрос о существовании вещественных участков дисперсионных кривых и докажем существование счетного числа частот, которым соответствуют решения, не зависящие от координаты $z$. Рассмотрим задачу относительно параметра $k$ при фиксированном $\gamma$, не обязательно равном нулю. При этом будем использовать систему уравнений относительно вектора $H$. Для сокращения обозначений будем считать, что $\varepsilon, \mu$ - скалярные функции. Сведем систему уравнений Максвелла к уравнению относительно магнитного поля:

$$
\operatorname{rot} \varepsilon^{-1} \operatorname{rot} H-k^{2} \mu H=0 .
$$

Зададим краевые условия

$$
\varepsilon^{-1} \operatorname{rot} H \times\left.\boldsymbol{n}\right|_{\partial Q}=0,\left.\quad H \boldsymbol{n}\right|_{\partial Q}=0
$$

и условия сопряжения

$$
\left.[H \times \boldsymbol{n}]\right|_{S}=0,\left.\quad[\mu H \boldsymbol{n}]\right|_{S}=0,\left.\quad\left[\varepsilon^{-1} \operatorname{rot} H \times \boldsymbol{n}\right]\right|_{S}=0 .
$$

Введем дополнительное условие

$$
\operatorname{div} \mu H=0 .
$$

Рассмотрим решения вида $H(x, y) e^{i \gamma z}$. Для векторов $H(x, y)$ получим систему

$$
\begin{gathered}
\operatorname{rot} \varepsilon^{-1} \operatorname{rot} H_{\perp}+\gamma^{2} \varepsilon^{-1} H_{\perp}+i \gamma \varepsilon^{-1} \operatorname{grad} H_{z}=k^{2} \mu H_{\perp}, \\
i \gamma \operatorname{div} \varepsilon^{-1} H_{\perp}-\operatorname{div} \varepsilon^{-1} \operatorname{grad} H_{z}=k^{2} \mu H_{z} .
\end{gathered}
$$


При этом необходимо рассматривать задачу на множестве векторов, для которых

$$
\operatorname{div} \mu H_{\perp}+i \gamma \mu H_{z}=0 .
$$

Легко видеть, что при любом вещественном $\gamma$ задача, рассматриваемая на множестве векторов, удовлетворяющих условию (6.7), сводится к задаче определения характеристических чисел самосопряженного компактного оператора. Справедлива

ЛЕмма 6.1. При любом вещественном $\gamma$ существует бесконечное число собственных значений задачи (6.1)-(6.4) с единственной точкой сәущения на бесконечности.

ДокАЗАТЕЛЬСтво. Введем полуторалинейные формы

$$
\begin{aligned}
a_{2}(H, \widetilde{H})=\left(\varepsilon^{-1} \operatorname{rot} H_{\perp}, \operatorname{rot} \widetilde{H}_{\perp}\right)_{L_{2}}+\left(\varepsilon^{-1} \operatorname{grad} H_{z}, \operatorname{grad} \widetilde{H}_{z}\right)_{L_{2}} \\
+i \gamma\left(\varepsilon^{-1} \widetilde{H}_{\perp}, \operatorname{grad} H_{z}\right)_{L_{2}}+i \gamma\left(\varepsilon^{-1} H_{\perp}, \operatorname{grad} \widetilde{H}_{z}\right)_{L_{2}} \\
+\gamma^{2}\left(\varepsilon^{-1} H_{\perp}, \widetilde{H}_{\perp}\right)_{L_{2}}+\left(\varepsilon^{-1} H_{\perp}, \widetilde{H}_{\perp}\right), \\
c_{2}(H, \widetilde{H})=(\mu H, \widetilde{H}) .
\end{aligned}
$$

Введем функциональное пространство

$$
\begin{aligned}
V_{2}=\left\{H_{\perp} \in H(\operatorname{rot}), H_{z} \in H^{1},\right. \\
\left.\quad\left(\mu H_{\perp}, \operatorname{grad} \tilde{\phi}\right)_{L_{2}}+i \gamma\left(\mu H_{z}, \tilde{\phi}\right)_{L_{2}}=0 \quad \forall \tilde{\phi} \in H^{1},\left.H_{\perp} n\right|_{\partial \Omega}=0\right\} .
\end{aligned}
$$

Рассмотрим обобщенную постановку задачи (6.1)-(6.4). Будем искать решения $H \in V_{2}$ и собственное значение $k$, удовлетворяющие задаче

$$
a_{2}(H, \widetilde{H})=\left(k^{2}+1\right) c(H, \widetilde{H}) \quad \forall \widetilde{H} \in V_{2} .
$$

УТВЕРЖДЕНИЕ 6.1. Пространство $V_{2}$ вложено в $\left(L_{2}\right)^{3}$ компактно.

ДокАзАТЕЛЬство аналогично доказательству теоремы 2.2 .

Форма $a_{2}(H, \widetilde{H})$ удовлетворяет неравенству

$$
a_{2}(H, H) \geqslant C(\mu H, H) .
$$

Следовательно, $a_{2}(H, \widetilde{H})$ определяет в пространстве $V_{2}$ скалярное произведение. Таким образом, по теореме Рисса существует самосопряженный оператор $T$, определяемый равенством

$$
a_{2}(H, H)=c(T H, H) .
$$

Оператор $H$, очевидно, обращается в нуль только на нулевом элементе. При этом в силу компактности вложения $V_{2}$ в $\left(L_{2}\right)^{3}$ оператор $T$ компактен. Таким образом, задача (6.1)-(6.4) сводится к спектральной задаче для компактного самосопряженного оператора

$$
H=\left(k^{2}+1\right) T H .
$$

Поэтому существует бесконечная последовательность $k_{n}^{2}$ с точкой сгущения в бесконечности. Лемма доказана. 
Поскольку $\gamma=0$, решение вида $H(x, y) e^{i \gamma z}$ не зависит от $z$. Таким образом, существует счетное число значений $k^{2}$, которым соответствуют решения, не зависящие от $z$, т. е. частот отсечки.

\section{Глава 2. Задача рассеяния в нерегулярном электромагнитном волноводе}

Настоящая глава посвящена исследованию задачи рассеяния в нерегулярном электромагнитном волноводе. Основной целью в данной главе является доказательство разрешимости краевой задачи для системы уравнений Максвелла с определенными условиями излучения в нерегулярном волноводе, а также доказательство существования ловушечных мод в случае отсутствия потерь в среде, заполняющей волновод. Таким образом, устанавливается неединственность решения задачи рассеяния при определенных значениях частоты.

\section{§ 7. Постановка задачи}

Рассмотрим задачу о дифракции электромагнитной волны на магнитнодиэлектрическом теле в волноводе.

Введем еще раз уравнения и краевые условия, которым должно удовлетворять решение. Задача рассматривается в цилиндре $Q=\{(x, y) \in \Omega$, $z \in(-\infty,+\infty)\}$, где $\Omega$ - односвязная ограниченная область с кусочно гладкой границей (рис. 4).

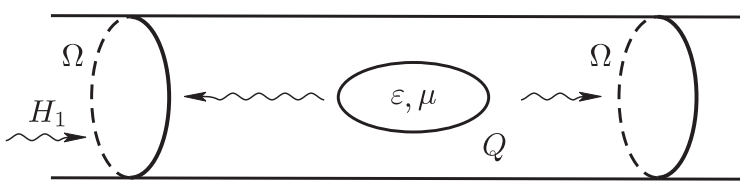

Рис. 4

Рассматривается система уравнений Максвелла

$$
\begin{gathered}
\operatorname{rot} E=i k \mu H, \quad \operatorname{rot} H=-i k \varepsilon E, \\
\operatorname{div} \varepsilon E=0, \quad \operatorname{div} \mu H=0 .
\end{gathered}
$$

Считаем, что $\varepsilon=I, \mu=I$ при $z<z_{1}$ и $z>z_{2}$. Диэлектрическая и магнитная проницаемости являются в общем случае эрмитовыми матрицами $\varepsilon=\varepsilon^{*}$, $\mu=\mu^{*}$, удовлетворяющими условиям ограниченности и положительной определенности

$$
\begin{aligned}
& \sum_{i}\left|\xi_{i}\right|^{2} \leqslant \sum_{i, j} \varepsilon_{i j} \xi_{i} \xi_{j}^{*} \leqslant \beta \sum_{i}\left|\xi_{i}\right|^{2}, \\
& \sum_{i}\left|\xi_{i}\right|^{2} \leqslant \sum_{i, j} \mu_{i j} \xi_{i} \xi_{j}^{*} \leqslant \beta \sum_{i}\left|\xi_{i}\right|^{2} \quad \forall \xi \in \mathbb{C}^{3} .
\end{aligned}
$$


ЗАмЕчАНИЕ 7.1. В ряде работ (см. [1]) рассматривался случай, когда матрицы $\varepsilon$ и $\mu$ диэлектрической и магнитной проницаемостей имеют вид

$$
\varepsilon=\varepsilon_{1}+i \varepsilon_{2} I, \quad \mu=\mu_{1}+i \mu_{2} I,
$$

где матрицы $\varepsilon_{1}$ и $\mu_{1}$ эрмитовы, $I$ - единичная матрица, а $\varepsilon_{2}, \mu_{2}$ - положительные константы, которые могут быть сколь угодно малыми. Введение малых величин $\varepsilon_{2}, \mu_{2}$ позволяет установить существование решения, однако применение принципа предельного поглощения в общем случае невозможно. Это связано с тем, что решение задачи при $\varepsilon_{2} \equiv 0, \mu_{2} \equiv 0$ может быть не единственным при определенных значениях частоты поля.

На границе цилиндра $Q$ поставим условия, соответствующие идеально проводящей стенке:

$$
E \times\left.\boldsymbol{n}\right|_{\partial Q}=0,\left.\quad \mu H \boldsymbol{n}\right|_{\partial Q}=0 .
$$

Считаем, что матрицы $\varepsilon$ и $\mu$ диэлектрической и магнитной проницаемостей отличны от единичной матрицы в области $V=\left\{(x, y) \in \Omega, z \in\left[z_{1}, z_{2}\right]\right\}$. На поверхностях $S$ разрыва функций $\varepsilon$ и $\mu$ введем условия сопряжения

$$
\begin{aligned}
{\left.[E \times \boldsymbol{n}]\right|_{S} } & =0, & & {\left.[H \times \boldsymbol{n}]\right|_{S}=0, } \\
\left.\varepsilon E \boldsymbol{n}\right|_{S} & =0, & & \left.\mu H \boldsymbol{n}\right|_{S}=0 .
\end{aligned}
$$

В качестве “условия на ребре" будем использовать условия

$$
E \in L_{2}^{\text {loc }}, \quad H \in L_{2}^{\text {loc }} .
$$

Уравнения (7.1), (7.2) с граничными условиями (7.3) и условиями сопряжения (7.4), (7.5) необходимо дополнить условиями излучения при $z \leqslant z_{1}$ и $z \geqslant z_{2}$ в полубесконечных областях, в которых поле удовлетворяет однородным уравнениям Максвелла

$$
\begin{gathered}
\operatorname{rot} E=i k H, \quad \operatorname{rot} H=-i k E, \\
\operatorname{div} E=0, \quad \operatorname{div} H=0 .
\end{gathered}
$$

Считаем, что из $-\infty$ на неоднородный участок волновода падает нормальная волна, вид которой мы конкретизируем позже.

В случае наличия поглощения в среде, заполняющей волновод, разрешимость задачи дифракции следует из энергетических оценок, полученных в работе [17]. Целью настоящей работы является доказательство разрешимости этой задачи в случае отсутствия в среде поглощения.

Сведем систему уравнений Максвелла к эквивалентной системе уравнений относительно магнитного поля:

$$
\operatorname{rot} \varepsilon^{-1} \operatorname{rot} H-k^{2} \mu H=0, \quad \operatorname{div} \mu H=0,
$$

с граничными условиями на боковой поверхности цилиндра $\partial Q$ :

$$
\operatorname{rot} H \times\left.\boldsymbol{n}\right|_{\partial Q}=0,\left.\quad H \boldsymbol{n}\right|_{\partial Q}=0,
$$

и условиями сопряжения

$$
\begin{gathered}
{\left.[H \times \boldsymbol{n}]\right|_{S}=0,} \\
{\left.\left[\varepsilon^{-1} \operatorname{rot} H \times \boldsymbol{n}\right]\right|_{S}=0 .}
\end{gathered}
$$


В качестве "условия на ребре" потребуем выполнения условий $H \in L_{2}^{\text {loc }}$, $\operatorname{rot} H \in L_{2}^{\text {loc }}$, т. е. условия ограниченности $L_{2}$-нормы векторов $H$ и $\operatorname{rot} H$ в любой конечной подобласти цилиндра $Q$.

Напомним [3], [19] определения некоторых функциональных пространств.

Введем пространство $H(\operatorname{rot}, V)=\left\{H \in L_{2}(V), \operatorname{rot} H \in L_{2}(V)\right\}$, которое является гильбертовым относительно нормы

$$
\|H\|_{H(\mathrm{rot})}^{2}=\|H\|_{L_{2}(V)}^{2}+\|\operatorname{rot} H\|_{L_{2}(V)}^{2} .
$$

Введем пространство $H(\operatorname{div}, V)=\left\{H \in L_{2}(V)\right.$, div $\left.H \in L_{2}(V)\right\}$, являющееся гильбертовым относительно нормы

$$
\|H\|_{H(\operatorname{div})}^{2}=\|H\|_{L_{2}(V)}^{2}+\|\operatorname{div} H\|_{L_{2}(V)}^{2} .
$$

Под условием $\left.H \boldsymbol{n}\right|_{\partial Q}=0$ для вектора $H \in H(\operatorname{div}, V)$ понимаем выполнение равенства

$$
(H, \operatorname{grad} \phi)_{L_{2}(V)}=-(\operatorname{div} H, \phi)_{L_{2}(V)} \quad \forall \phi \in H^{1}(V),\left.\quad \phi\right|_{\partial \Omega_{i}}=0, \quad i=1,2,
$$

где $\Omega_{i}$ - сечения цилиндра плоскостями $z=z_{i}, i=1,2$.

Перейдем к постановке условий излучения, следуя [1]. Пусть в областях $Q_{1}=\left\{(x, y) \in \Omega, z<z_{1}\right\}$ и $Q_{2}=\left\{(x, y) \in \Omega_{2}, z>z_{2}\right\}$ вектор $H$ удовлетворяет уравнению

$$
\operatorname{rot} \operatorname{rot} H-k^{2} H=0 \text {, }
$$

граничным условиям

$$
\left.H \boldsymbol{n}\right|_{\partial Q_{i}}=0, \quad \operatorname{rot} H \times\left.\boldsymbol{n}\right|_{\partial Q_{i}}=0, \quad i=1,2,
$$

и условиям $H \in L_{2}^{\text {loc }}, \operatorname{rot} H \in L_{2}^{\text {loc }}$. Тогда $\operatorname{rot} \operatorname{rot} H \in L_{2}^{\text {loc }}$.

Умножая уравнение (7.10) на произвольный достаточно гладкий вектор $\widetilde{H}$, отличный от нуля только при $z$, принадлежащем некоторому конечному отрезку, получим

$$
(\operatorname{rot} H, \operatorname{rot} \widetilde{H})_{L_{2}\left(Q_{i}\right)}=k^{2}(H, \widetilde{H})_{L_{2}\left(Q_{i}\right)} .
$$

УТВЕРЖДЕНИЕ 7.1. Пусть $\chi_{n}, \psi_{n}$ - ортонормированные собственные функиии соответственно задач

$$
\begin{aligned}
& -\Delta_{\perp} \chi_{n}=\lambda_{1 n} \chi_{n},\left.\quad \chi_{n}\right|_{\partial \Omega}=0, \\
& -\Delta_{\perp} \psi_{n}=\lambda_{2 n} \psi_{n},\left.\quad \frac{\partial \psi_{n}}{\partial \boldsymbol{n}}\right|_{\partial \Omega}=0, \\
& \left(\chi_{n}, \chi_{m}\right)_{L_{2}(\Omega)}=\delta_{n m}, \quad\left(\psi_{n}, \psi_{m}\right)_{L_{2}(\Omega)}=\delta_{n m} .
\end{aligned}
$$

Тогда векторы $H \in L_{2}(V) u \operatorname{rot} H \in L_{2}(V)$ при почти всех z представимы в виде сходяшихся в $L_{2}(\Omega)$ рядов:

$$
\begin{array}{r}
H=\sum_{n=1}^{\infty} \overline{\bar{Z}}_{2 n} \operatorname{grad}_{\perp} \psi_{n}+\sum_{n=1}^{\infty} Z_{1 n} \operatorname{rot} \chi_{n} e_{z}+\sum_{n=1}^{\infty} \overline{\bar{Z}}_{3 n} \psi_{n} e_{z}, \\
\operatorname{rot} H=\sum_{n=1}^{\infty} \bar{Z}_{2 n} \operatorname{rot} \psi_{n} e_{z}+\sum_{n=1}^{\infty} \bar{Z}_{1 n} \operatorname{grad}_{\perp} \chi_{n}+\sum_{n=1}^{\infty} \lambda_{1 n} \bar{Z}_{3 n} \chi_{n} e_{z},
\end{array}
$$

zдe

$$
Z_{\text {in }} \in L_{2}\left[z_{1}, z_{2}\right], \quad \bar{Z}_{\text {in }} \in L_{2}[0,1], \quad i=1,2,3 .
$$


Это утверждение следует из полноты системы векторов $\operatorname{grad}_{\perp} \psi_{n}, \operatorname{rot} \chi_{n} e_{z}$ в $\left(L_{2}(\Omega)\right)^{2}$, полноты каждой из систем функций $\chi_{n}, \psi_{n}$ в $L_{2}(\Omega)$ и равенств $\left(\operatorname{rot} \chi_{n} e_{z}, \operatorname{rot} \chi_{m} e_{z}\right)_{L_{2}(\Omega)}=\lambda_{1 n} \delta_{n m}, \quad\left(\operatorname{grad}_{\perp} \psi_{n}, \operatorname{grad}_{\perp} \psi_{m}\right)_{L_{2}(\Omega)}=\lambda_{2 n} \delta_{n m}$.

ЗАмЕчАниЕ 7.2. Поскольку функции $\psi_{n}, \chi_{n}$ удовлетворяют граничным условиям $(7.13),(7.14)$, то $\operatorname{grad}_{\perp} \psi_{n}, \operatorname{rot} \chi_{n} e_{z}$ удовлетворяют граничному условию $\left.H \boldsymbol{n}\right|_{\partial \Omega}=0$.

УтВеРЖДЕНИЕ 7.2. Пусть $\operatorname{div} H=0$ в области $V$. Тогда вектор $H$ представим в следующем виде:

$$
H=\sum_{n=1}^{\infty} Z_{2 n}^{\prime} \operatorname{grad}_{\perp} \psi_{n}+\sum_{n=1}^{\infty} Z_{1 n} \operatorname{rot} \chi_{n} e_{z}+\sum_{n=1}^{\infty} Z_{2 n} \psi_{n} e_{z}
$$

где ряды сходятся в $L_{2}(V)$.

Действительно, в силу условия $\operatorname{div} H=0$ имеет место равенство

$$
\left(H, \operatorname{grad} \widetilde{Z}_{n} \psi_{n}\right)_{L_{2}(V)}=\left(H_{\perp}, \widetilde{Z}_{n} \operatorname{grad} \psi_{n}\right)_{L_{2}(V)}+\left(H_{z}, \widetilde{Z}_{n}^{\prime} \psi_{n}\right)_{L_{2}(V)}=0
$$

для любого $\widetilde{Z}_{n} \in C_{0}^{\infty}$. Отсюда следует, что $\lambda_{2 n} \overline{\bar{Z}}_{2 n}=\overline{\bar{Z}}_{3 n}^{\prime}$. Обозначим $Z_{2 n}=$ $\frac{1}{\lambda_{2 n}} \overline{\bar{Z}}_{3 n}$. Тогда $\overline{\bar{Z}}_{2 n}=Z_{2 n}^{\prime}$.

УтВеРЖДЕНИЕ 7.3. Пусть $H \in H(\operatorname{rot}), \operatorname{div} H=0$. Тогда

$$
\operatorname{rot} H=-\sum_{n=1}^{\infty}\left(Z_{2 n}^{\prime \prime}-\lambda_{2 n} Z_{2 n}\right) \operatorname{rot} \psi_{n} e_{z}+\sum_{n=1}^{\infty}\left(Z_{1 n}^{\prime} \operatorname{grad}_{\perp} \chi_{n}+\lambda_{1 n} Z_{1 n} \chi_{n} e_{z}\right)
$$

Воспользуемся тем, что для любого вектора $\widetilde{H}=\widetilde{Z}_{n} \chi_{n} e_{z}, \widetilde{Z}_{n} \in C_{0}^{\infty}$, справедливо равенство

$$
\left(\operatorname{rot} H, \widetilde{Z}_{n} \chi_{n} e_{z}\right)_{L_{2}(V)}=\left(H, \widetilde{Z}_{n} \operatorname{rot} \chi_{n} e_{z}\right)_{L_{2}(V)} .
$$

Отсюда следуют равенства $\bar{Z}_{3 n}=\lambda_{1 n} Z_{1 n}, \bar{Z}_{1 n}=\lambda_{1 n} Z_{1 n}^{\prime}$. Выбирая вектор $\widetilde{H}=\operatorname{rot} \operatorname{rot}\left(\widetilde{Z}_{n} \psi_{n} e_{z}\right)$, получим равенство

$$
\left(\operatorname{rot} H, \operatorname{rot}\left(\widetilde{Z}_{n} \psi_{n} e_{z}\right)\right)_{L_{2}(V)}=\left(H, \operatorname{rot} \operatorname{rot}\left(\widetilde{Z}_{n} \psi_{n} e_{z}\right)\right)_{L_{2}(V)} .
$$

Отсюда имеем

$$
\bar{Z}_{2 n}=-Z_{2 n}^{\prime \prime}+\lambda_{2 n} Z_{2 n}
$$

При этом $\left(Z_{1 n}, Z_{1 n}^{\prime}, Z_{2 n}, Z_{2 n}^{\prime}, Z_{2 n}^{\prime \prime}-\lambda_{2 n} Z_{2 n}\right) \in L_{2}\left[z_{1}, z_{2}\right]$.

ЗАмЕчАНИЕ 7.3. Из утверждений 7.2 и 7.3 следует, что для векторов $H$, удовлетворяющих условиям $H \in H(\operatorname{rot}, V), \operatorname{div} H=0,\left.H \boldsymbol{n}\right|_{\partial Q}=0$, возможно определение величины $\left(H_{\perp}, \operatorname{grad}_{\perp} \psi_{n}\right)_{L_{2}\left(\Omega_{i}\right)}$, под которой мы подразумеваем $\lambda_{2 n} Z_{2 n}^{\prime}\left(z_{i}\right), i=1,2$.

Пусть вектор $H$ удовлетворяет уравнению (7.12). Выберем в качестве вектоpa $\widetilde{H}$ вектор $\widetilde{Z}_{n} \operatorname{rot} \chi_{n} e_{z}$. Считаем для определенности, что $n>N_{1}$. Выберем $\widetilde{Z}_{n} \in C^{\infty}, Z_{n} \equiv 0, z<z_{2}-\tau$. В результате для $Z_{1 n}$ получим

$$
\left(Z_{1 n}^{\prime}, \widetilde{Z}_{n}^{\prime}\right)_{L_{2}}-\left(\lambda_{1 n}-k^{2}\right)\left(Z_{1 n}, \widetilde{Z}_{n}\right)+\frac{1}{\lambda_{1 n}} \gamma_{n} Z_{1 n}\left(z_{2}\right) \widetilde{Z}_{n}\left(z_{2}\right)=0 .
$$


Следовательно, $Z_{1 n}$ удовлетворяет уравнению $Z_{1 n}^{\prime \prime}-\left(\lambda_{1 n}-k^{2}\right) Z_{1 n}=0$.

Обозначим $\gamma_{1 n}=\sqrt{\lambda_{1 n}-k^{2}}$. Отсюда следует, что $Z_{1 n}(z)$ представимо в области $\left(z_{2}-\tau, z_{2}\right)$ в виде

$$
Z_{1 n}=a_{1 n} e^{-\gamma_{1 n} z}+a_{2 n} e^{\gamma_{2 n} z}
$$

Обозначим $\gamma_{2 n}=\sqrt{\lambda_{2 n}-k^{2}}$. Рассматривая в качестве вектора $\widetilde{H}$ вектор $\widetilde{Z}_{n} \psi_{n} e_{z}$, получим, что $Z_{2 n}$ имеет вид

$$
Z_{2 n}=c_{1 n} e^{-\gamma_{2 n} z}+c_{2 n} e^{\gamma_{2 n} z}
$$

В качестве условий излучения используем парциальные условия излучения А. Г. Свешникова [16] в областях $z<z_{1}$ и $z>z_{2}$ в виде

$$
\begin{aligned}
& Z_{1 n}=a_{1 n} e^{-\gamma_{1 n} z}, \quad z>z_{2}, \quad Z_{1 n}=a_{2 n} e^{\gamma_{1 n} z}+\delta_{n m} A e^{i\left|\gamma_{1 m}\right| z}, \quad z<z_{1}, \\
& Z_{2 n}=c_{1 n} e^{-\gamma_{2 n} z}, \quad z>z_{2}, \quad Z_{2 n}=c_{2 n} e^{\gamma_{2 n} z}, \quad z<z_{1} \text {. }
\end{aligned}
$$

ЗАмечаниЕ 7.4. Считаем далее везде, что $k^{2} \neq \lambda_{1 n}, k^{2} \neq \lambda_{2 n}$, т. е. $k^{2}$ не совпадает ни с одной из частот отсечки полого волновода.

Считаем, что из $-\infty$ падает нормальная волна вида $H=A \operatorname{rot}\left(\chi_{m} e_{z}\right) e^{i \gamma_{1 m} z}$, где $A$ - константа, $m \leqslant N_{1}$.

Условия излучения можно сформулировать как требование представления вектора $H$ при $z<z_{1}+\tau$ и $z>z_{2}-\tau$ в виде сходящегося в $\left(L_{2}(\Omega)\right)^{3}$ ряда:

$$
\begin{aligned}
H= & \sum_{n=1}^{\infty} a_{n} \operatorname{rot}\left(\chi_{n} e_{z}\right) e^{-\gamma_{1 n} z} \\
& +\sum_{n=1}^{\infty} c_{n}\left(-\gamma_{2 n} \operatorname{grad}_{\perp} \psi_{n}+\lambda_{2 n} \psi_{n} e_{z}\right) e^{-\gamma_{2 n} z}, \quad z>z_{2}, \\
H= & \sum_{n=1}^{\infty} b_{n} \operatorname{rot}\left(\chi_{n} e_{z}\right) e^{\gamma_{1 n} z}+\sum_{n=1}^{\infty} d_{n}\left(\gamma_{2 n} \operatorname{grad}_{\perp} \psi_{n}+\lambda_{2 n} \psi_{n} e_{z}\right) e^{\gamma_{2 n} z} \\
& +A e^{i\left|\gamma_{1 m}\right| z} \operatorname{rot} \chi_{m} e_{z}, \quad z<z_{1} .
\end{aligned}
$$

В случае если вектор $H$ представим в областях $z<z_{1}+\tau$ и $z>z_{2}-\tau$ в виде сходящихся в $L_{2}(\Omega)$ рядов, то вектор $\operatorname{rot} H \in\left(L_{2}\right)^{3, \text { loc }}$ можно разложить в сходящиеся в $L_{2}(\Omega)$ ряды:

$$
\begin{aligned}
& \operatorname{rot} H=\sum_{n=1}^{\infty} a_{n}\left(\gamma_{1 n} \operatorname{grad}_{\perp} \chi_{n}+\lambda_{1 n} \chi_{n} e_{z}\right) e^{-\gamma_{1 n} z} \\
& \quad+k^{2} \sum_{n=1}^{\infty} c_{n} \operatorname{rot}\left(\psi_{n} e_{z}\right) e^{\gamma_{2 n} z}, \quad z>z_{2}, \\
& \operatorname{rot} H=\sum_{n=1}^{\infty} b_{n}\left(\gamma_{1 n} \operatorname{grad}_{\perp} \chi_{n}+\lambda_{1 n} \chi_{n} e_{z}\right) e^{\gamma_{1 n} z}+k^{2} \sum_{n=1}^{\infty} d_{n} \operatorname{rot}\left(\psi_{n} e_{z}\right) e^{-\gamma_{2 n} z} \\
& \quad+A e^{i\left|\gamma_{1 m}\right| z}\left(i \gamma_{1 m} \operatorname{grad}_{\perp} \chi_{m}+\lambda_{1 m} \chi_{m} e_{z}\right), \quad z<z_{1} .
\end{aligned}
$$




\section{§ 8. Вариационная постановка задачи}

Перейдем к вариационной постановке задачи дифракции. Сформулируем еще раз рассматриваемую задачу. Будем искать решения уравнений

$$
\begin{gathered}
\operatorname{rot} \varepsilon^{-1} \operatorname{rot} H-k^{2} \mu H=0, \\
\operatorname{div} \mu H=0
\end{gathered}
$$

с краевыми условиями

$$
\left.\mu H \boldsymbol{n}\right|_{\partial Q}=0, \quad \varepsilon^{-1} \operatorname{rot} H \times\left.\boldsymbol{n}\right|_{\partial Q}=0
$$

и условиями сопряжения на поверхностях разрыва $S$ диэлектрической и магнитной проницаемостей:

$$
\left.[H \times \boldsymbol{n}]\right|_{S}=0,\left.\quad\left[\varepsilon^{-1} \operatorname{rot} H \times \boldsymbol{n}\right]\right|_{S}=0 .
$$

Условия излучения зададим как требования представления поля $H$ в виде сходящихся в $L_{2}(\Omega)$ рядов при $z<z_{1}$ и $z>z_{2}$ :

$$
\begin{aligned}
H= & \sum_{n=1}^{\infty} c_{n} \operatorname{rot}\left(\chi_{n} e^{-\gamma_{1 n} z} e_{z}\right)+\sum_{n=1}^{\infty} d_{n} \operatorname{rot} \operatorname{rot}\left(\psi_{n} e^{-\gamma_{2 n} z} e_{z}\right), \quad z>z_{2}, \\
H=\sum_{n=1}^{\infty} a_{n} \operatorname{rot}\left(\chi_{n} e^{\gamma_{1 n} z} e_{z}\right) & \\
& +\sum_{n=1}^{\infty} b_{n} \operatorname{rot} \operatorname{rot}\left(\psi_{n} e^{\gamma_{2 n} z} e_{z}\right)+A \operatorname{rot}\left(\chi_{m} e_{z}\right) e^{i \gamma_{1 m} z}, \quad z<z_{1} .
\end{aligned}
$$

Умножим уравнение (8.1) на произвольный кусочно гладкий вектор $\widetilde{H}$, удовлетворяющий граничным условиям, условиям сопряжения и условиям излучения, и проинтегрируем по области $V$ :

$$
\int_{V}\left(\operatorname{rot} \varepsilon^{-1} \widetilde{H}^{*} \operatorname{rot} H-k^{2} \widetilde{H}^{*} \mu H\right) d v=0 .
$$

Интегрируя по частям (считаем, что это возможно), получим

$$
\begin{aligned}
& \int_{V}\left(\varepsilon^{-1} \operatorname{rot} H \operatorname{rot} \widetilde{H}^{*}-k^{2} \mu H \widetilde{H}^{*}\right) d v \\
&+\sum_{i=1}^{2} \sum_{n=1}^{\infty} \gamma_{n} \frac{1}{\lambda_{1 n}}\left(H_{\perp}, \operatorname{rot} \chi_{n} e_{z}\right)_{L_{2}\left(\Omega_{2}\right)}\left(\widetilde{H}_{\perp}, \operatorname{rot} \chi_{n} e_{z}\right)_{L_{2}\left(\Omega_{2}\right)}^{*} \\
&-\sum_{i=1}^{2} \sum_{n=1}^{\infty} \frac{k^{2}}{\lambda_{2 n} \gamma_{2 n}}\left(H_{\perp}, \operatorname{grad}_{\perp} \psi_{n}\right)_{L_{2}\left(\Omega_{i}\right)}\left(\widetilde{H}_{\perp}, \operatorname{grad}_{\perp} \psi_{n}\right)_{L_{2}\left(\Omega_{i}\right)}^{*} \\
&=2 i\left|\gamma_{1 m}\right| A e^{i\left|\gamma_{1 m}\right| z}\left(\operatorname{rot} \chi_{m} e_{z}, \widetilde{H}\right)_{L_{2}\left(\Omega_{1}\right)} .
\end{aligned}
$$

Под обобщенным решением задачи (8.1)-(8.4) будем понимать решение уравнения (8.5). Вопрос о том, в каком функциональном пространстве необходимо рассматривать задачу (8.5), требует специального изучения и будет исследован далее. 


\section{§9. Существование решения задачи рассеяния в волноводе}

Рассмотрим [35] обобщенную постановку задачи (7.6)-(7.9), (7.18), (7.19). Обозначим

$$
\gamma_{1 n}=\left\{\begin{array}{ll}
\sqrt{k^{2}-\lambda_{1 n}}, & n=1, \ldots, N_{1}, \\
\sqrt{\lambda_{1 n}-k^{2}}, & n>N_{1},
\end{array} \quad \gamma_{2 n}= \begin{cases}\sqrt{k^{2}-\lambda_{2 n}}, & n=1, \ldots, N_{2}, \\
\sqrt{\lambda_{2 n}-k^{2}}, & n>N_{2},\end{cases}\right.
$$

при условии $k^{2}>\lambda_{1 n}, n=1, \ldots, N_{1}, k^{2}>\lambda_{2 n}, n=1, \ldots, N_{2}$.

Будем искать решение уравнения

$$
\begin{aligned}
&\left(\varepsilon^{-1} \operatorname{rot} H\right., \operatorname{rot} \widetilde{H})_{L_{2}(V)}-k^{2}(\mu H, \widetilde{H})_{L_{2}(V)} \\
&-\sum_{i=1}^{2} \sum_{n=1}^{N_{1}} i \gamma_{1 n} \frac{1}{\lambda_{1 n}}\left(H_{\perp}, \operatorname{rot} \chi_{n} e_{z}\right)_{L_{2}\left(\Omega_{i}\right)}\left(\widetilde{H}_{\perp}, \operatorname{rot} \chi_{n} e_{z}\right)_{L_{2}\left(\Omega_{i}\right)}^{*} \\
&+\sum_{i=1}^{2} \sum_{n=N_{1}+1}^{\infty} \gamma_{1 n} \frac{1}{\lambda_{1 n}}\left(H_{\perp}, \operatorname{rot} \chi_{n} e_{z}\right)_{L_{2}\left(\Omega_{i}\right)}\left(\widetilde{H}_{\perp}, \operatorname{rot} \chi_{n} e_{z}\right)_{L_{2}\left(\Omega_{i}\right)}^{*} \\
&-\sum_{i=1}^{2} \sum_{n=1}^{N_{2}} \frac{i k^{2}}{\lambda_{2 n} \gamma_{2 n}}\left(H_{\perp}, \operatorname{grad}_{\perp} \psi_{n}\right)_{L_{2}\left(\Omega_{i}\right)}\left(\widetilde{H}_{\perp}, \operatorname{grad}_{\perp} \psi_{n}\right)_{L_{2}\left(\Omega_{i}\right)}^{*} \\
&-\sum_{i=1}^{2} \sum_{n=N_{2}+1}^{\infty} \frac{k^{2}}{\lambda_{2 n} \gamma_{2 n}}\left(H_{\perp}, \operatorname{grad}_{\perp} \psi_{n}\right)_{L_{2}\left(\Omega_{i}\right)}\left(\widetilde{H}_{\perp}, \operatorname{grad}_{\perp} \psi_{n}\right)_{L_{2}\left(\Omega_{i}\right)}^{*} \\
&=2 i \gamma_{1 m} A e^{i \gamma_{1 m} z}\left(\operatorname{rot} \chi_{m} e_{z}, \widetilde{H}\right)_{L_{2}\left(\Omega_{2}\right)}
\end{aligned}
$$

Введем полуторалинейные формы

$$
\begin{aligned}
a(H, \widetilde{H})=\left(\varepsilon^{-1} \operatorname{rot} H, \operatorname{rot} \widetilde{H}\right)_{L_{2}(V)} & \\
& +\sum_{i=1}^{2} \sum_{n=N_{1}+1}^{\infty} \gamma_{1 n} \frac{1}{\lambda_{1 n}}\left(H_{\perp}, \operatorname{rot} \chi_{n} e_{z}\right)_{L_{2}\left(\Omega_{i}\right)}\left(\widetilde{H}_{\perp}, \operatorname{rot} \chi_{n} e_{z}\right)_{L_{2}\left(\Omega_{i}\right)}^{*}, \\
b(H, \widetilde{H})=- & k^{2}(\mu H, \widetilde{H})_{L_{2}(V)} \\
& -\sum_{i=1}^{2} \sum_{n=1}^{N_{1}} i \gamma_{1 n} \frac{1}{\lambda_{1 n}}\left(H_{\perp}, \operatorname{rot} \chi_{n} e_{z}\right)_{L_{2}\left(\Omega_{i}\right)}\left(\widetilde{H}_{\perp}, \operatorname{rot} \chi_{n} e_{z}\right)_{L_{2}\left(\Omega_{i}\right)}^{*} \\
& -\sum_{n=1}^{N_{2}} \frac{i k^{2}}{\lambda_{2 n} \gamma_{2 n}}\left(H_{\perp}, \operatorname{grad}_{\perp} \psi_{n}\right)_{L_{2}\left(\Omega_{i}\right)}\left(\widetilde{H}_{\perp}, \operatorname{grad}_{\perp} \psi_{n}\right)_{L_{2}\left(\Omega_{i}\right)}^{*} \\
& -\sum_{n=N_{2}+1}^{\infty} \frac{k^{2}}{\lambda_{2 n} \gamma_{2 n}}\left(H_{\perp}, \operatorname{grad}_{\perp} \psi_{n}\right)_{L_{2}\left(\Omega_{i}\right)}\left(\widetilde{H}_{\perp}, \operatorname{grad}_{\perp} \psi_{n}\right)_{L_{2}\left(\Omega_{i}\right)}^{*}
\end{aligned}
$$

и линейную форму

$$
l(\widetilde{H})=2 i \gamma_{1 m} A\left(\operatorname{rot} \chi_{m} e_{z}, \widetilde{H}\right)_{L_{2}\left(\Omega_{2}\right)} .
$$

В обозначениях (9.2), (9.3) уравнение (9.1) имеет вид

$$
a(H, \widetilde{E})+b(H, \widetilde{E})=l(\widetilde{H}) .
$$


Для обобщенной постановки задачи (7.6)-(7.19) необходимо ввести векторное функциональное пространство, в котором будем рассматривать задачу. В областях $z \leqslant z_{1}+\tau$ и $z \geqslant z_{2}-\tau$ компоненты магнитного поля $H$, представимого в виде рядов (7.18), (7.19), связаны соотношениями

$$
\begin{aligned}
& \left(H_{\perp}, \operatorname{grad}_{\perp} \psi_{n}\right)_{L_{2}\left(\Omega_{2}\right)}= \begin{cases}i \gamma_{2 n}\left(H_{z}, \psi_{n}\right)_{L_{2}\left(\Omega_{2}\right)}, & n=1, \ldots, N_{2}, \\
-\gamma_{2 n}\left(H_{z}, \psi_{n}\right)_{L_{2}\left(\Omega_{2}\right)}, & n>N_{2},\end{cases} \\
& \left(H_{\perp}, \operatorname{grad}_{\perp} \psi_{n}\right)_{L_{2}\left(\Omega_{1}\right)}= \begin{cases}-i \gamma_{2 n}\left(H_{z}, \psi_{n}\right)_{L_{2}\left(\Omega_{1}\right)} & n=1, \ldots, N_{2}, \\
\gamma_{2 n}\left(H_{z}, \psi_{n}\right)_{L_{2}\left(\Omega_{1}\right)}, & n>N_{2} .\end{cases}
\end{aligned}
$$

Введем функциональное пространство, на элементы которого наложим условия (9.6):

$$
\begin{aligned}
\mathcal{V}=\{ & H \in H(\operatorname{rot}, V), \operatorname{div} \mu H=0,\left.\mu H \boldsymbol{n}\right|_{\partial Q}=0, \\
& \sum_{i=1}^{2} \sum_{n=N_{1}+1}^{\infty} \frac{1}{\lambda_{1 n}} \gamma_{1 n}\left(H_{\perp}, \operatorname{rot} \chi_{n} e_{z}\right)_{L_{2}\left(\Omega_{i}\right)}^{2}<\infty \\
& \left(H_{\perp}, \operatorname{grad}_{\perp} \psi_{n}\right)_{L_{2}\left(\Omega_{2}\right)}=i \gamma_{2 n}\left(H_{z}, \psi_{n}\right)_{L_{2}\left(\Omega_{2}\right)}, \quad n=1, \ldots, N_{2}, \\
& \left(H_{\perp}, \operatorname{grad}_{\perp} \psi_{n}\right)_{L_{2}\left(\Omega_{2}\right)}=-\gamma_{2 n}\left(H_{z}, \psi_{n}\right)_{L_{2}\left(\Omega_{2}\right)}, \quad n>N_{2}, \\
& \left(H_{\perp}, \operatorname{grad}_{\perp} \psi_{n}\right)_{L_{2}\left(\Omega_{1}\right)}=-i \gamma_{2 n}\left(H_{z}, \psi_{n}\right)_{L_{2}\left(\Omega_{1}\right)}, \quad n=1, \ldots, N_{2}, \\
& \left.\left(H_{\perp}, \operatorname{grad}_{\perp} \psi_{n}\right)_{L_{2}\left(\Omega_{1}\right)}=\gamma_{2 n}\left(H_{z}, \psi_{n}\right)_{L_{2}\left(\Omega_{1}\right)}, \quad n>N_{2}\right\} .
\end{aligned}
$$

ЗАМЕчАНИЕ 9.1. В силу утверждений (7.2) и 7.3 для вектора $H$, удовлетворяющего условиям $H \in H(\operatorname{rot}, V), \operatorname{div} \mu H=0,\left.\mu H \boldsymbol{n}\right|_{\partial Q}=0$, определено значение $\left(H_{\perp}, \operatorname{grad}_{\perp} \psi_{n}\right)_{L_{2}\left(\Omega_{i}\right)}, i=1,2$.

ОПРЕДЕЛЕНИЕ 9.1. Будем называть вектор $H \in \mathcal{V}$ обобщеннъм решением задачи (7.6)-(7.19), если он удовлетворяет уравнению $(9.5)$ для любого $\widetilde{H} \in \mathcal{V}$.

Докажем существование обобщенного решения задачи (7.6)-(7.19), т. е. решения задачи (9.5).

Полуторалинейная форма $a(H, \widetilde{H})$ задает скалярное произведение в пространстве $\mathcal{V}$. Пространство $\mathcal{V}$ является гильбертовым с нормой, порождаемой скалярным произведением $a(H, \widetilde{H})$. Проверки требует, очевидно, только полнота пространства $\mathcal{V}$, доказательство которой осуществляется аналогично доказательству, приведенному в работе [42].

УТВеРЖДЕНИЕ 9.1. Для элементов пространства $\mathcal{V}$ определена полуторалинейная форма $b(H, \widetilde{H})$. При фиксированном $H \in \mathcal{V}$ она определяет ограниченный функиионал в $\mathcal{V}$.

ДокАЗАТЕЛЬСТво утверждения следует из приведенной ниже леммы 9.2.

Таким образом, в силу теоремы Рисса [28] существуют ограниченный в пространстве $\mathcal{V}$ оператор $T$ и вектор $F$ такие, что

$$
\begin{gathered}
a(T H, \widetilde{H})=b(H, \widetilde{H}), \\
a(F, \widetilde{H})=l(\widetilde{H}) .
\end{gathered}
$$


Уравнение (9.5) сводится к уравнению

$$
H+T H=F
$$

Докажем, что оператор $T$ является компактным в пространстве $\mathcal{V}$. Отсюда будет следовать существование решения задачи (9.9). В то же время решение задачи может быть не единственным.

УТВЕРЖДЕНИЕ 9.2. Пусть при некотором $k$, которое по условию является вещественным, существует нетривиальное решение однородного уравнения (9.9). Тогда ядро сопряженного уравнения ортогонально правой частиуравнения (9.5), поскольку оно удовлетворяет условиям

$$
\left(H_{\perp}, \operatorname{rot} \chi_{n} e_{z}\right)_{L_{2}\left(\Omega_{i}\right)}=0, \quad n=1, \ldots, N_{2}, \quad i=1,2 .
$$

Действительно, если существует решение $H$ однородного сопряженного уравнения $H+T^{*} H=0$, то оно удовлетворяет уравнению $(b(H, H))^{*}=0$, что приводит к равенству

$$
-\sum_{i=1}^{2} \sum_{n=1}^{N_{1}} i \frac{1}{\lambda_{1 n}} \gamma_{1 n}\left|\left(H_{\perp}, \operatorname{rot} \chi_{n} e_{z}\right)_{L_{2}\left(\Omega_{2}\right)}\right|^{2}=0 .
$$

Учитывая, что $a(F, H)=l(H)$, приходим к ортогональности правой части уравнения (9.9) ядру сопряженной задачи относительно скалярного произведения $a(H, \widetilde{H})$.

Введем пространство

$$
W=\left\{H \in L_{2}(V), \sum_{i=1}^{2} \sum_{n=N_{2}+1}^{\infty} \frac{1}{\lambda_{2 n} \gamma_{2 n}}\left|\left(H_{\perp}, \operatorname{grad}_{\perp} \psi_{n}\right)_{L_{2}\left(\Omega_{i}\right)}\right|^{2}\right\}
$$

которое является гильбертовым относительно нормы

$$
\|H\|_{W}^{2}=\|H\|_{L_{2}(V)}^{2}+\sum_{i=1}^{2} \sum_{n=N_{2}+1}^{\infty} \frac{1}{\lambda_{2 n} \gamma_{2 n}}\left|\left(H_{\perp}, \operatorname{grad}_{\perp} \psi_{n}\right)_{L_{2}\left(\Omega_{i}\right)}\right|^{2} .
$$

ТЕОРема 9.1. Пространство $\mathcal{V}$ компактно вложено в $W$.

ДокАзАТЕльство теоремы разобьем на доказательства двух лемм.

ЛЕмма 9.1. Пространство $\mathcal{V}$ вложено в $\left(L_{2}(V)\right)^{3}$ компактно.

ЗАмЕчАниЕ 9.2. Доказать теорему 9.1 достаточно для случая $\mu=I$. В работе [20] доказан данный фундаментальный факт для теоремы о компактности вложения пространства векторов, удовлетворяющих условиям $H \in H(\mathrm{rot}, V)$, $\mu H \in H_{0}(\operatorname{div}, V)$, в $L_{2}(V)$. Доказательство того, что достаточно рассматривать случай $\mu=I$, для пространства $\mathcal{V}$ полностью аналогично. Особенностью применяемого в настоящей работе метода является единообразное рассмотрение вопроса о компактности вложения для векторов электрического и магнитного полей, удовлетворяющих разным граничным условиям. 
ДоКАЗАТЕЛЬСтво ЛЕммы 9.1. Рассмотрим ограниченную в пространстве $\mathcal{V}$ последовательность векторов $H_{k}:\left\|H_{k}\right\|_{\mathcal{V}}^{2}<C$. Представим векторы $H_{k}$ и rot $H_{k}$ в виде

$$
\begin{aligned}
H_{k}=\sum_{n=1}^{\infty} Z_{2 k_{n}}^{\prime} \operatorname{grad}_{\perp} & \psi_{n}+\sum_{n=1}^{\infty} Z_{1 k_{n}} \operatorname{rot} \chi_{n} e_{z}+\sum_{n=1}^{\infty} \lambda_{2 n} Z_{2 k_{n}} \psi_{n} e_{z}, \\
\operatorname{rot} H_{k}=- & \sum_{n=1}^{\infty}\left(Z_{2 k_{n}}^{\prime \prime}-\lambda_{2 n} Z_{2 k_{n}}\right) \operatorname{rot} \psi_{n} e_{z} \\
& +\sum_{n=1}^{\infty}\left(Z_{1 k_{n}}^{\prime} \operatorname{grad}_{\perp} \chi_{n}+\lambda_{1 n} Z_{1 k_{n}} \chi_{n} e_{z}\right)
\end{aligned}
$$

где $Z_{1 k_{n}} \in L_{2}, Z_{1 k_{n}}^{\prime} \in L_{2}, Z_{2 k_{n}} \in L_{2}, Z_{2 k_{n}}^{\prime} \in L_{2}, Z_{2 k_{n}}^{\prime \prime}-\lambda_{n} Z_{2 k_{n}} \in L_{2}$.

Справедливы равенства

$$
\begin{gathered}
\left\|H_{k}\right\|_{L_{2}(V)}^{2}=\sum_{n=1}^{\infty}\left(\lambda_{2 n}\left\|Z_{2 k_{n}}^{\prime}\right\|^{2}+\lambda_{2 n}^{2}\left\|Z_{2 k_{n}}\right\|^{2}\right)+\sum_{n=1}^{\infty} \lambda_{1 n}\left\|Z_{1 k_{n}}^{\prime}\right\|^{2} \\
\left\|\operatorname{rot} H_{k}\right\|_{L_{2}(V)}^{2}=\sum_{n=1}^{\infty} \lambda_{2 n}\left\|Z_{2 k_{n}}^{\prime \prime}-\lambda_{2 n} Z_{2 k_{n}}\right\|^{2} \\
+\sum_{n=1}^{\infty}\left(\lambda_{1 n}\left\|Z_{1 k_{n}}^{\prime}\right\|^{2}+\lambda_{1 n}^{2}\left\|Z_{1 k_{n}}\right\|^{2}\right),
\end{gathered}
$$

где $\|Z\|=\|Z\|_{L_{2}\left[z_{1}, z_{2}\right]}$. В силу условий $Z_{1 k_{n}}^{\prime} \in L_{2}, Z_{2 k_{n}}^{\prime \prime} \in L_{2}$ определены величины $Z_{1 k_{n}}\left(z_{i}\right), Z_{2 k_{n}}^{\prime}\left(z_{i}\right), i=1,2$.

В силу выполнения условий

$$
\begin{aligned}
& \left(H_{\perp_{k}}, \operatorname{grad}_{\perp} \psi_{n}\right)_{L_{2}\left(\Omega_{2}\right)}= \begin{cases}i \gamma_{2 n}\left(H_{z_{k}}, \psi_{n}\right)_{L_{2}\left(\Omega_{2}\right)}, & n=1, \ldots, N_{2}, \\
-\gamma_{2 n}\left(H_{z_{k}}, \psi_{n}\right)_{L_{2}\left(\Omega_{2}\right)}, & n>N_{2},\end{cases} \\
& \left(H_{\perp_{k}}, \operatorname{grad}_{\perp} \psi_{n}\right)_{L_{2}\left(\Omega_{1}\right)}= \begin{cases}-i \gamma_{2 n}\left(H_{z_{k}}, \psi_{n}\right)_{L_{2}\left(\Omega_{1}\right)}, & n=1, \ldots, N_{2}, \\
-\gamma_{2 n}\left(H_{z_{k}}, \psi_{n}\right)_{L_{2}\left(\Omega_{1}\right)}, & n>N_{2},\end{cases}
\end{aligned}
$$

справедливы условия

$$
\begin{aligned}
& Z_{2 k_{n}}^{\prime}\left(z_{2}\right)= \begin{cases}i \gamma_{2 n} Z_{2 k_{n}}\left(z_{2}\right), & n=1, \ldots, N_{2}, \\
-\gamma_{2 n} Z_{2 k_{n}}\left(z_{2}\right), & n>N_{2}\end{cases} \\
& Z_{2 k_{n}}^{\prime}\left(z_{1}\right)= \begin{cases}-i \gamma_{2 n} Z_{2 k_{n}}\left(z_{1}\right), & n=1, \ldots, N_{2}, \\
\gamma_{2 n} Z_{2 k_{n}}\left(z_{1}\right), & n>N_{2} .\end{cases}
\end{aligned}
$$

Таким образом, имеет место равенство

$$
\begin{aligned}
& \sum_{n=1}^{\infty} \lambda_{2 n}\left\|Z_{2 k_{n}}^{\prime \prime}-\lambda_{2 n} Z_{2 k_{n}}\right\|^{2}=\sum_{n=1}^{\infty}\left(\lambda_{2 n}\left\|Z_{2 k_{n}}^{\prime \prime}\right\|^{2}+2 \lambda_{2 n}^{2}\left\|Z_{2 k_{n}}^{\prime}\right\|^{2}\right. \\
& \left.\quad+\lambda_{2 n}^{3}\left\|Z_{2 k_{n}}\right\|^{2}\right)+\sum_{i=1}^{2} \sum_{n=N_{1}+1}^{\infty} \lambda_{1 n}^{2} \gamma_{1 n}\left|Z_{2 k_{n}}\left(z_{i}\right)\right|^{2}
\end{aligned}
$$


В результате имеем

$$
\begin{aligned}
& \left\|\operatorname{rot} H_{k}\right\|_{L_{2}(V)}^{2}=\sum_{n=1}^{\infty}\left(\lambda_{2 n}\left\|Z_{2 k_{n}}^{\prime \prime}\right\|^{2}+2 \lambda_{2 n}^{2}\left\|Z_{2 k_{n}}^{\prime}\right\|^{2}+\lambda_{2 n}^{3}\left\|Z_{2 k_{n}}\right\|^{2}\right) \\
& \quad+\sum_{i=1}^{2} \sum_{n=N_{2}+1}^{\infty} \lambda_{2 n}^{2} \gamma_{2 n}\left|Z_{2 k_{n}}\left(z_{i}\right)\right|^{2}+\sum_{n=1}^{\infty}\left(\lambda_{1 n}\left\|Z_{1 k_{n}}^{\prime}\right\|^{2}+\lambda_{1 n}^{2}\left\|Z_{1 k_{n}}\right\|^{2}\right) .
\end{aligned}
$$

Легко видеть, что из любой последовательности, ограниченной по норме

$$
\sum_{n=1}^{\infty}\left(\lambda_{1 n}\left\|Z_{1 k_{n}}^{\prime}\right\|^{2}+\lambda_{1 n}^{2}\left\|Z_{1 k_{n}}\right\|^{2}\right)
$$

можно выделить подпоследовательность, фундаментальную относительно нормы $\sum_{n=1}^{\infty} \lambda_{1 n}\left\|Z_{1 k_{n}}\right\|^{2}$.

Поскольку $\sum_{n=1}^{\infty} \lambda_{1 n}^{2}\left\|Z_{1 k_{n}}\right\|^{2}<C$, то имеет место неравенство

$$
\sum_{n=N_{1}+1}^{\infty} \lambda_{1 n}\left\|Z_{1 k_{n}}\right\|^{2}<\lambda_{1 N_{1}} \sum_{n=N_{1}+1}^{\infty} \lambda_{1 n}\left\|Z_{1 k_{n}}\right\|^{2}<\sum_{n=N_{1}+1}^{\infty} \lambda_{1 n}\left\|Z_{1 k_{n}}\right\|^{2}<C .
$$

Так как

$$
\sum_{n=N_{1}+1}^{\infty} \lambda_{1 n}\left\|Z_{1 k_{n}}\right\|^{2}<\frac{C}{\lambda_{1, N_{1}+1}}
$$

и $\lambda_{1, N_{1}+1}=O\left(N_{1}+1\right)$ при $N_{1} \rightarrow \infty$, то $\sum_{n=N_{1}+1}^{\infty} \lambda_{1 n}\left\|Z_{1 k_{n}}\right\|^{2}$ сколь угодно мала при больших $N_{1}$, не зависящих от $k$. Для конечной суммы

$$
\sum_{n=1}^{N_{1}} \lambda_{1 n}\left\|Z_{1 k_{n}}\right\|^{2}
$$

применима теорема Релиха [23]. В результате справедливо утверждение о возможности выделить фундаментальную подпоследовательность относительно нормы $\sum_{n=1}^{\infty} \lambda_{1 n}\left\|Z_{1 k_{n}}\right\|^{2}$. Аналогично, из любой последовательности, ограниченной по норме

$$
\sum_{n=1}^{\infty}\left(\lambda_{2 n}\left\|Z_{2 k_{n}}^{\prime \prime}\right\|^{2}+2 \lambda_{2 n}^{2}\left\|Z_{2 k_{n}}^{\prime}\right\|^{2}+\lambda_{2 n}^{3}\left\|Z_{2 k_{n}}\right\|^{2}\right)+\sum_{i=1}^{2} \sum_{n=N_{2}+1}^{\infty} \lambda_{2 n}^{2} \gamma_{2 n}\left|Z_{2 k_{n}}\left(z_{i}\right)\right|^{2}
$$

можно выделить сходящуюся подпоследовательность по норме

$$
\sum_{n=1}^{\infty}\left(\lambda_{2 n}\left\|Z_{2 k_{n}}^{\prime}\right\|^{2}+\lambda_{2 n}^{2}\left\|Z_{2 k_{n}}\right\|^{2}\right) .
$$

Таким образом, пространство $\mathcal{V}$ вложено в $\left(L_{2}(V)\right)^{3}$ компактно.

Лемма 9.2. Из любой ограниченной по норме $\mathcal{V}$ последовательности можно выделить сходящуюся подпоследовательность следов по норме

$$
\sum_{i=1}^{2} \sum_{n=N_{2}+1}^{\infty} \frac{1}{\lambda_{2 n} \gamma_{2 n}}\left|\left(H_{\perp}, \operatorname{grad}_{\perp} \psi_{n}\right)_{L_{2}(\Omega)}\right|^{2} .
$$


ДокАЗАТЕЛЬСТво сводится к установлению равенства

$$
\sum_{i=1}^{2} \sum_{n=N_{2}+1}^{\infty} \frac{1}{\lambda_{2 n} \gamma_{2 n}}\left|\left(H_{\perp_{k}}, \operatorname{grad}_{\perp} \psi_{n}\right)_{L_{2}(\Omega)}\right|^{2}=\sum_{i=1}^{2} \sum_{n=N_{2}+1}^{\infty} \lambda_{2 n} \gamma_{2 n}\left|Z_{2 k_{n}}\left(z_{2}\right)\right|^{2}
$$

и ограниченности суммы

$$
\sum_{i=1}^{2} \sum_{n=N_{2}+1}^{\infty} \lambda_{2 n}^{2} \gamma_{2 n}\left|Z_{2 k_{n}}\left(z_{2}\right)\right|^{2}
$$

что следует из доказательства теоремы 9.1.

Отсюда имеем, что из любой ограниченной последовательности по норме пространства $\mathcal{V}$ можно выделить фундаментальную подпоследовательность по указанной полунорме.

Из ограниченности в $L_{2}$ норм $\left\|Z_{2 k_{n}}^{\prime}\right\|,\left\|Z_{2 k_{n}}^{\prime \prime}\right\|, n=1, \ldots, N_{2},\left\|Z_{1 k_{n}}\right\|,\left\|Z_{1 k_{n}}^{\prime}\right\|$, $n=1, \ldots, N_{1}$, следует, что из последовательности функций, ограниченных по норме пространства $\mathcal{V}$, можно выделить фундаментальную подпоследовательность по полунорме

$$
\sum_{i=1}^{2} \sum_{n=1}^{N_{2}}\left|Z_{2 k_{n}}^{\prime}\left(z_{i}\right)\right|^{2}+\sum_{i=1}^{2} \sum_{n=1}^{N_{1}}\left|Z_{1 k_{n}}\left(z_{i}\right)\right|^{2}
$$

или по полунорме

$$
\sum_{i=1}^{2} \sum_{n=1}^{N_{2}} \frac{1}{\lambda_{2 n} \gamma_{2 n}}\left(H_{\perp}, \operatorname{grad}_{\perp} \psi_{n}\right)_{L_{2}\left(\Omega_{i}\right)}^{2}+\sum_{i=1}^{2} \sum_{n=1}^{N_{1}} \frac{1}{\lambda_{1 n}} \gamma_{1 n}\left(H_{\perp}, \operatorname{rot} \chi_{n} e_{z}\right)_{L_{2}\left(\Omega_{i}\right)}^{2}
$$

В результате получаем, что оператор $T$ компактный.

Таким образом, справедлива

Теорема 9.2. Существует решение задачи (9.5).

ЗАмЕчАнИЕ 9.3. Будем считать, что существует такое малое $\tau$, что в областях $\left(z_{1}, z_{1}+\tau\right)$ и $\left(z_{2}-\tau, z_{2}\right)$ коэффициенты системы уравнений имеют вид $\varepsilon=I, \mu=I$. Решение задачи (9.5) имеет вид (7.18), (7.19) при $z>z_{2}-\tau$ и $z<z_{1}+\tau$. В самом деле, в качестве вектора $\widetilde{\widetilde{H}}$ можно выбрать как произвольный вектор вида $\widetilde{H}=\widetilde{Z}(z) \psi_{n} e_{z}$, где $\widetilde{Z}\left(z_{2}\right)=0, \widetilde{Z}(z)=0, z \leqslant z_{2}-\tau$, так и вектор $\widetilde{H}=\widetilde{Z}(z) \operatorname{rot} \chi_{n} e_{z}$, где $\widetilde{Z}\left(z_{2}\right)=0, \widetilde{Z}(z)=0, z \leqslant z_{2}-\tau$. Отсюда, учитывая результаты, полученные в $\S 7$, имеем, что решение представимо в виде (7.18), (7.19).

\section{§ 10. Дискретный спектр задачи рассеяния}

Дискретный спектр задачи рассеяния на диэлектрическом теле может быть непуст. В задачах акустики и теории упругости подобный вопрос исследовался во многих работах (см., например, [39]-[41]). Для доказательства будем использовать метод, аналогичный методу, применяемому в работах [36]-[38]. Несложно доказать непустоту дискретного спектра в случае, когда $\mu=I, \varepsilon=\varepsilon(x, y, z) I$ и $1 \leqslant \varepsilon \leqslant \varepsilon_{\max }$. Считаем, что $\varepsilon>1$ при $(x, y, z) \in V_{1}, \varepsilon=1$ при $(x, y, z) \notin V$, 
область $V_{1}$, занятая рассеивателем, ограничена, $V_{1} \subset\left\{(x, y) \in \Omega, z \in\left[z_{1}, z_{2}\right]\right\}$. Рассматривается задача в цилиндре $Q$ :

$$
\begin{gathered}
\operatorname{rot} \varepsilon^{-1} \operatorname{rot} H-k^{2} H=0, \quad \operatorname{div} H=0, \\
\left.H \boldsymbol{n}\right|_{\partial Q}=0, \quad \varepsilon^{-1} \operatorname{rot} H \times\left.\boldsymbol{n}\right|_{\partial Q}=0, \quad H \in L_{2}(Q) .
\end{gathered}
$$

ОПРЕДЕЛЕНИЕ 10.1. Обобщенным решением задачи (10.1) будем называть вектор $H \in H(\operatorname{rot}, Q), \operatorname{div} H=0,\left.H \boldsymbol{n}\right|_{\partial Q}=0$, удовлетворяющий уравнению

$$
\left(\varepsilon^{-1} \operatorname{rot} H, \operatorname{rot} \widetilde{H}\right)_{L_{2}(Q)}=k^{2}(\mu H, \widetilde{H})_{L_{2}(Q)} \quad \forall \widetilde{H} \in H(\operatorname{rot}, Q) .
$$

ОПРЕДЕЛЕНИЕ 10.2. Будем говорить, что дискретный спектр задачи рассеяния непуст, если существует обобщенное решение $H \neq 0, k^{2} \in \mathbb{R}$ задачи (10.1).

Для доказательства непустоты дискретного спектра рассмотрим спектральную задачу вида

$$
\begin{aligned}
& \left(\varepsilon^{-1} \operatorname{rot} H, \operatorname{rot} \widetilde{H}\right)_{L_{2}(V)}+\sum_{i=1}^{2} \sum_{n=1}^{\infty} \gamma_{1 n} \frac{1}{\lambda_{1 n}}\left(H, \operatorname{rot} \chi_{n} e_{z}\right)_{L_{2}\left(\Omega_{i}\right)}\left(\widetilde{H}, \operatorname{rot} \chi_{n} e_{z}\right)_{L_{2}\left(\Omega_{i}\right)} \\
& \quad-\sum_{i=1}^{2} \sum_{n=1}^{\infty} \frac{k^{2}}{\gamma_{2 n} \lambda_{2 n}}\left(H, \operatorname{grad}_{\perp} \psi_{n}\right)_{L_{2}\left(\Omega_{i}\right)}\left(\widetilde{H}, \operatorname{grad}_{\perp} \psi_{n}\right)_{L_{2}\left(\Omega_{i}\right)} \\
& =k^{2}(H, \widetilde{H})_{L_{2}(V)}
\end{aligned}
$$

Из существования решения задачи (10.2) следует существование решения в цилиндре $Q$. Для доказательства существования собственного значения $k \in \mathbb{R}$ и собственной функции $H$ задачи (10.2) рассмотрим вспомогательную задачу относительно нового спектрального параметра $\nu$ :

$$
\begin{aligned}
& \left(\varepsilon^{-1} \operatorname{rot} H, \operatorname{rot} \widetilde{H}\right)_{L_{2}(V)}+\sum_{i=1}^{2} \sum_{n=1}^{\infty} \gamma_{1 n} \frac{1}{\lambda_{1 n}}\left(H, \operatorname{rot} \chi_{n} e_{z}\right)_{L_{2}\left(\Omega_{i}\right)}\left(\widetilde{H}, \operatorname{rot} \chi_{n} e_{z}\right)_{L_{2}\left(\Omega_{i}\right)} \\
& \quad-\sum_{i=1}^{2} \sum_{n=1}^{\infty} \frac{k^{2}}{\gamma_{2 n} \lambda_{2 n}}\left(H, \operatorname{grad}_{\perp} \psi_{n}\right)_{L_{2}\left(\Omega_{i}\right)}\left(\widetilde{H}, \operatorname{grad}_{\perp} \psi_{n}\right)_{L_{2}\left(\Omega_{i}\right)}=\nu(k)(H, \widetilde{H})_{L_{2}(V)} .
\end{aligned}
$$

Считаем, что $k$ изменяется на промежутке $\left(0, \lambda_{21}\right]$, где $\lambda_{21}-$ первое ненулевое собственное значение задачи

$$
-\Delta_{\perp} \psi=\lambda_{2} \psi,\left.\quad \frac{\partial \psi}{\partial \boldsymbol{n}}\right|_{\partial \Omega}=0,
$$

рассматриваемой в поперечном сечении $\Omega$. Будем рассматривать задачу в пространстве $\mathcal{V}$. 


\section{Справедливо}

УТВЕРЖДЕНИЕ 10.1. Билинейная форма

$$
\begin{aligned}
a(A, \widetilde{A}) & +C(A, \widetilde{A})_{L_{2}(V)}=\left(\varepsilon^{-1} \operatorname{rot} H, \operatorname{rot} \widetilde{H}\right)_{L_{2}(V)} \\
& +\sum_{i=1}^{2} \sum_{n=1}^{\infty} \gamma_{1 n} \frac{1}{\lambda_{1 n}}\left(H, \operatorname{rot} \chi_{n} e_{z}\right)_{L_{2}\left(\Omega_{i}\right)}\left(\widetilde{H}, \operatorname{rot} \chi_{n} e_{z}\right)_{L_{2}\left(\Omega_{i}\right)} \\
& -\sum_{i=1}^{2} \sum_{n=1}^{\infty} \frac{k^{2}}{\gamma_{2 n} \lambda_{2 n}}\left(H, \operatorname{grad}_{\perp} \psi_{n}\right)_{L_{2}\left(\Omega_{i}\right)}\left(\widetilde{H}, \operatorname{grad}_{\perp} \psi_{n}\right)_{L_{2}\left(\Omega_{i}\right)}+C(A, \widetilde{A})_{L_{2}(V)},
\end{aligned}
$$

где $C$ - достаточно большая константа, порождает скалярное произведение в пространстве $\mathcal{V}$.

Необходимо доказать только то, что

$$
a(H, H)+C(H, H)>0 .
$$

Для этого достаточно рассмотреть равенство

$$
\sum_{i=1}^{2} \sum_{n=1}^{\infty} \frac{k^{2}}{\gamma_{2 n} \lambda_{2 n}}\left(H_{\perp}, \operatorname{grad}_{\perp} \psi_{n}\right)_{L_{2}\left(\Omega_{i}\right)}^{2}=\sum_{i=1}^{2} \sum_{n=1}^{\infty} k^{2} \lambda_{2 n} \gamma_{2 n}\left|Z_{2 n}\left(z_{i}\right)\right|^{2} .
$$

В силу соотношений

$$
\begin{gathered}
\lambda_{2 n} \gamma_{2 n}\left|Z_{2 n}\left(z_{i}\right)\right|^{2} \leqslant C_{1} \lambda_{2 n}^{2}\left\|Z_{2 n}\right\|^{2}+C_{2} \gamma_{2 n}^{2}\left\|Z_{2 n}^{\prime}\right\|^{2} \leqslant C_{1} \lambda_{2 n}^{2}\left\|Z_{2 n}\right\|^{2}+C_{3} \lambda_{2 n}\left\|Z_{2 n}^{\prime}\right\|^{2}, \\
\|H\|_{L_{2}(V)}^{2}=\sum_{i=1}^{2} \sum_{n=1}^{\infty} \lambda_{2 n}\left\|Z_{2 n}^{\prime}\right\|^{2}+\sum_{i=1}^{2} \sum_{n=1}^{\infty} \lambda_{2 n}^{2}\left\|Z_{2 n}\right\|^{2},
\end{gathered}
$$

можно выбрать $C$ таким образом, чтобы выполнялось условие (10.4).

УТВЕРЖДЕНИЕ 10.2. Существует по крайней мере одно собственное значение спектральной задачи (10.3), которое определяется равенством

$$
\begin{aligned}
\nu_{1}=\inf _{H \in V} & \frac{1}{(H, H)_{L_{2}(V)}}\left(\varepsilon^{-1} \operatorname{rot} H, \operatorname{rot} H\right)_{L_{2}(V)} \\
& +\sum_{i=1}^{2} \sum_{n=1}^{\infty} \gamma_{1 n} \frac{1}{\lambda_{1 n}}\left(H, \operatorname{rot} \chi_{n} e_{z}\right)_{L_{2}\left(\Omega_{i}\right)}^{2}-\sum_{i=1}^{2} \sum_{n=1}^{\infty}\left(H, \operatorname{grad}_{\perp} \psi_{n}\right)_{L_{2}\left(\Omega_{i}\right)}^{2} .
\end{aligned}
$$

Это утверждение справедливо в силу компактности вложения пространства $V$ в $L_{2}(V)$.

УТВЕРЖДЕНИЕ 10.3. Собственное значение $\nu_{1}(k)$ убывает монотонно $u$ непрерывно на отрезке $\left[0, \lambda_{21}\right]$.

ДокАЗАТЕЛЬСтво непрерывности и монотонности $\nu_{1}(k)$ аналогично доказательству, приведенному в работе [42]. Следовательно, если $\nu_{1}\left(\lambda_{21}\right)<\lambda_{21}$, то существует собственное значение спектральной задачи (10.2). Действительно, рассмотрим отношение Релея при $H=\psi_{1}(x, y) e_{z}$ и $k=\lambda_{21}$. В результате получим

$$
\nu_{1}<\frac{\left(\varepsilon^{-1} \operatorname{rot} \operatorname{rot} \psi_{1} e_{z}, \operatorname{rot} \operatorname{rot} \psi_{1} e_{z}\right)_{L_{2}(V)}}{\left(\operatorname{rot} \psi_{1} e_{z}, \operatorname{rot} \psi_{1} e_{z}\right)_{L_{2}(V)}}<\frac{\left(\Delta \psi_{1}, \Delta \psi_{1}\right)_{L_{2}(V)}}{\left(\operatorname{rot} \psi_{1} e_{z}, \operatorname{rot} \psi_{1} e_{z}\right)_{L_{2}(V)}}=\lambda_{21} .
$$


Отсюда следует, что справедливо

УтвеРжДЕниЕ 10.4. Существует решение спектральной задачи (10.2).

Построим решение в бесконечной области $Q$. Для этого продолжим его в область $z>z_{2}$ по формуле

$$
\begin{aligned}
H= & \sum_{n=1}^{\infty} \frac{1}{\lambda_{1 n}}\left(H, \operatorname{rot} \chi_{n} e_{z}\right)_{L_{2}\left(\Omega_{2}\right)} \operatorname{rot}\left(\chi_{n} e_{z}\right) e^{-\gamma_{1 n} z} \\
& +\sum_{n=1}^{\infty} \frac{1}{\lambda_{2 n}}\left(H, \operatorname{grad}_{\perp} \psi_{n}\right)_{L_{2}\left(\Omega_{2}\right)}\left(-\gamma_{2 n} \operatorname{grad}_{\perp} \psi_{n}+\lambda_{2 n} \psi_{n} e_{z}\right) e^{-\gamma_{2 n} z}
\end{aligned}
$$

и в область $z<z_{1}$ аналогичным образом:

$$
\begin{aligned}
H=\sum_{n=1}^{\infty} & \frac{1}{\lambda_{1 n}}\left(H, \operatorname{rot} \chi_{n} e_{z}\right)_{L_{2}\left(\Omega_{2}\right)} \operatorname{rot}\left(\chi_{n} e_{z}\right) e^{\gamma_{1 n} z} \\
& +\sum_{n=1}^{\infty} \frac{1}{\lambda_{2 n}}\left(H, \operatorname{grad}_{\perp} \psi_{n}\right)_{L_{2}\left(\Omega_{2}\right)}\left(\gamma_{2 n} \operatorname{grad}_{\perp} \psi_{n}+\lambda_{2 n} \psi_{n} e_{z}\right) e^{\gamma_{2 n} z}
\end{aligned}
$$

Легко видеть, что $H \in L_{2}(Q)$ и $\operatorname{rot} H \in L_{2}(Q), \operatorname{div} H=0,\left.H \boldsymbol{n}\right|_{\partial Q}=0$. Проверка этого при $z<z_{1}$ и $z>z_{2}$ осуществляется непосредственным вычислением. На сечениях $\Omega_{i}$ для следов $H \times\left.\boldsymbol{n}\right|_{\partial \Omega_{i}},\left.H \boldsymbol{n}\right|_{\partial \Omega_{i}}$ выполняются условия $\left.[H \times \boldsymbol{n}]\right|_{z=z_{i}}=0,\left.[H \boldsymbol{n}]\right|_{z=z_{i}}=0, i=1,2$. Проверка первого условия осуществляется рассмотрением соответствующего ряда при $z=z_{i}$. Второе условие следует из равенств

$$
\begin{aligned}
\left(H, \operatorname{grad}_{\perp} \psi_{n}\right)_{L_{2}\left(\Omega_{2}\right)} & =-\gamma_{2 n}\left(H_{z}, \psi_{n}\right)_{L_{2}\left(\Omega_{2}\right)}, \\
\left(H, \operatorname{grad}_{\perp} \psi_{n}\right)_{L_{2}\left(\Omega_{1}\right)} & =\gamma_{2 n}\left(H_{z}, \psi_{n}\right)_{L_{2}\left(\Omega_{1}\right)} .
\end{aligned}
$$

Несложно проверить, что таким образом построенный вектор $H$ является решением задачи

$$
\left(\varepsilon^{-1} \operatorname{rot} H, \operatorname{rot} \widetilde{H}\right)_{L_{2}(Q)}-k^{2}(H, \widetilde{H})_{L_{2}(Q)}=0 \quad \forall \widetilde{H} \in H(\operatorname{rot}, Q)
$$

и, следовательно, справедливо

УтВЕРЖДЕНИЕ 10.5. Существует обобщенное решение задачи (10.1).

\section{§11. О компактности вложения пространства $H(\operatorname{rot}, V) \cap H_{0}(\operatorname{div}, V)$ в $L_{2}(V)$}

Для доказательства теоремы 9.1 о компактности вложения применяется метод, который может быть использован единообразно для доказательства компактности вложения как пространства $H_{0}(\operatorname{rot}, V) \cap H(\operatorname{div}, V)$, так и пространства $H(\operatorname{rot}, V) \cap H_{0}(\operatorname{div}, V)$ в $L_{2}(V)$ для определенного частного случая областей. Наиболее важные результаты, относящиеся к теоремам вложения в областях с негладкой границей для векторных полей, получены в работах [19]-[21]. В настоящей работе мы рассматриваем другой метод доказательства этих теорем вложения. Этот метод, в отличие от методов, используемых 
в работах [19]-[21], применим единообразно к доказательству соответствующих теорем вложения.

Пусть $V$ - цилиндр конечной высоты: $V=\left\{(x, y) \in \Omega, z \in\left[z_{1}, z_{2}\right]\right\}$. Тогда справедлива

Теорема 11.1. Пространство $H(\operatorname{rot}, V) \cap H_{0}(\operatorname{div}, V)$ вложено в $L_{2}(V)$ компактно.

ДокАЗАТЕЛЬство. Как следует из [19], достаточно рассмотреть случай, когда векторы $H$ удовлетворяют условию $\operatorname{div} H=0$. Используем представление вектора $H$ в виде ряда:

$$
H=\sum_{n=1}^{\infty} Z_{2 n}^{\prime} \operatorname{grad}_{\perp} \psi_{n}+\sum_{n=1}^{\infty} Z_{1 n} \operatorname{rot} \chi_{n} e_{z}+\sum_{n=1}^{\infty} \lambda_{2 n} Z_{2 n} \psi_{n} e_{z}
$$

и учтем, что $Z_{2 n}\left(z_{i}\right)=0, i=1,2$. В остальном доказательство не отличается от доказательства теоремы 9.1. Учитывая граничные условия, имеем

$$
\begin{gathered}
\|H\|_{H(\mathrm{rot}, V)}^{2}=\sum_{n=1}^{\infty} \lambda_{1 n}\left\|Z_{1 n}^{\prime}\right\|^{2}+\sum_{n=1}^{\infty} \lambda_{1 n}^{2}\left\|Z_{1 n}\right\|^{2}+\sum_{n=1}^{\infty}\left\|-Z_{2 n}^{\prime \prime}+\lambda_{2 n} Z_{2 n}\right\|^{2} \\
=\sum_{n=1}^{\infty} \lambda_{1 n}\left\|Z_{1 n}^{\prime}\right\|^{2}+\sum_{n=1}^{\infty} \lambda_{1 n}^{2}\left\|Z_{1 n}\right\|^{2} \\
\quad+\sum_{n=1}^{\infty} \lambda_{2 n}\left\|Z_{2 n}^{\prime \prime}\right\|^{2}+2 \sum_{n=1}^{\infty} \lambda_{2 n}^{2}\left\|Z_{2 n}^{\prime}\right\|^{2}+\sum_{n=1}^{\infty} \lambda_{2 n}^{3}\left\|Z_{2 n}\right\|^{2}
\end{gathered}
$$

Учитывая, что

$$
\|H\|_{L_{2}(V)}^{2}=\sum_{n=1}^{\infty} \lambda_{1 n}\left\|Z_{1 n}\right\|^{2}+\sum_{n=1}^{\infty} \lambda_{2 n}\left\|Z_{2 n}^{\prime}\right\|^{2}+\sum_{n=1}^{\infty} \lambda_{2 n}^{2}\left\|Z_{2 n}\right\|^{2},
$$

приходим к доказательству утверждения теоремы.

ЗАмечАниЕ 11.1. Теорема 11.1, очевидно, может быть распространена на некоторые области более широкого класса. В частности, к областям, на которые распространяется теорема, относятся области с границами, описываемыми в работе [21].

\section{Список литературы}

1. А. С. Ильинский, В. В. Кравцов, А. Г. Свешников, Математические модели электродинамики, Высш. школа, М., 1991.

2. Л.И. Седов, Механика сплошной средъ, т. 1, Наука, М., 1970; англ. пер.: L. I. Sedov, Mechanics of continuous media, Vol. 1, 2, Ser. Theoret. Appl. Mech., 4, World Scientific, River Edge, NJ, 1997.

3. Г. Дюво, Ж.-Л. Лионс, Неравенства в механике и физике, Наука, М., 1980; пер. c фp.: G. Duvaut, J.-L. Lions, Les inéquations en mécanique et en physique, Dunod, Paris, 1972.

4. А. С. Зильберглейт, Ю. И. Копилевич, Спектральная теория регулярных волноводов, Изд-во Физ.-тех. ин-та, Л., 1983. 
5. Ю. Г. Смирнов, "Метод операторных пучков в краевых задачах сопряжения для системы эллиптических уравнений”, Дифферени. уравн., 27:1 (1991), 140-147; англ. пер.: Yu. G. Smirnov, "Operator-bundle method for conjugation boundary-value problems for a system of elliptic equations", Differ. Equ., 27:1 (1991), 112-118.

6. Ю. Г. Смирнов, "О полноте системы собственных и присоединенных волн частично заполненного волновода с нерегулярной границей”, ДАН СССР, 297:4 (1987), 829-832; англ. пер.: Yu. G. Smirnov, "Completeness of the system of eigen- and associated waves of a partially filled waveguide with an irregular boundary", Soviet Physics Dokl., 32:12 (1987), 963-964.

7. Ю. Г. Смирнов, "Применение метода операторных пучков в задаче о собственных волнах частично заполненного волновода", ДАН СССР, 312:3 (1990), 597-599; англ. пер.: Yu. G. Smirnov, "The application of the operator pencil method in a problem concerning the natural waves of a partially filled wave guide", Soviet Physics Dokl., 35:5 (1990), 430-431.

8. П.Е. Краснушкин, Е.И. Моисеев, "О возбуждении вынужденных колебаний в слоистом радиоволноводе", ДАН СССР, 264:5 (1982), 1123-1127; англ. пер.: P. E. Krasnushkin, E. I. Moiseev, "The excitation of forced oscillations in a stratified radio-waveguide", Soviet Physics Dokl., 27:6 (1982), 458-460.

9. И.Ц. Гохберг, М.Г. Крейн, Введение в теорию линейных несамосопряженных операторов в гильбертовом пространстве, Наука, М., 1965; англ. пер.: I. C. Gohberg, M. G. Krein, Introduction to the theory of linear nonselfadjoint operators, Amer. Math. Soc., Providence, RI, 1969.

10. А. Г. Костюченко, М.Б. Оразов, “Задача о колебаниях упругого полуцилиндра и связанные с ней самосопряженные операторные пучки", Тр. сем. им. И. Г. Петровского, 6 (1981), 97-146.

11. А.А. Самарский, А.Н. Тихонов, "О возбуждении радиоволноводов. 1", Журн. техн. физики, 17:11 (1947), 1284-1296.

12. А.А. Самарский, А.Н. Тихонов, "О возбуждении радиоволноводов. 2", Журн. техн. физики, 17:12 (1947), 1431-1440.

13. А. А. Самарский, А. Н. Тихонов, “О представлении поля в волноводе в виде суммы полей ТЕ и ТМ”, Журн. техн. физики, 18:7 (1948), 959-970.

14. А. Гараджаев, М. Б. Оразов, "Об условиях затухания решений и принципе излучения для одного дифференциального уравнения с операторными коэффициентами на полуоси", Дифферени. уравн., 19:6 (1983), 944-954; англ. пер.: A. Garadzhaev, M. B. Orazov, "Conditions for the attenuation of solutions and the radiation principle for a differential equation with operational coefficients in the plane", Differ. Equ., 19:6 (1983), 682-691.

15. А. Г. Свешников, "Принцип излучения", ДАН СССР, 73:5 (1950), 917-920.

16. А.Г. Свешников, "Принцип предельного поглощения для волноводов", ДАН CCCP, 80:3 (1951), 345-347.

17. А. Г. Свешников, "К обоснованию метода расчета электромагнитных полей в нерегулярных волноводах", ЖВМ и МФ, 3:2 (1963), 314-326.

18. А. Г. Свешников, "Обоснование методов исследования распространения электромагнитных колебаний в волноводах с анизотропным заполнением”, ЖKВМ и $М \Phi$, 3:5 (1963), 935-955.

19. М.Ш. Бирман, М.З. Соломяк, “Оператор Максвелла в областях с негладкой границей”, Сиб. матем. журн., 28:1 (1987), 23-36; англ. пер.: M.Sh. Birman, M. Z. Solomyak, "Maxwell operator in regions with nonsmooth boundaries", Siberian Math. J., 28:1 (1987), 12-24.

20. М.Ш. Бирман, М. З. Соломяк, “Самосопряженный оператор Максвелла в произвольных областях", Алгебра и анализ, 1:1 (1989), 96-110; англ. пер.: M. Sh. Birman, M. Z. Solomyak, "The self-adjoint Maxwell operator in arbitrary domains", Leningrad Math. J., 1:1 (1990), 99-115. 
21. М.Ш. Бирман, М. З. Соломяк, "Главные особенности электрической составляющей электромагнитного поля в областях с экранами", Алгебра и анализ, 5:1 (1993), 143-159; англ. пер.: M. Sh. Birman, M. Z. Solomyak, "On the main singularities of the electric component of the electro-magnetic field in regions with screens", St. Petersburg Math. J., 5:1 (1994), 125-139.

22. F. Rellich, "Das Eigenwertproblem von $\Delta u+\lambda u=0$ in Halbröhren", Studies and essays presented to R. Courant on his 60 th birthday, Inters. Publ., New York, 1948, 329-344.

23. О.А. Ладыженская, Краевые задачи математической физики, Наука, М., 1973; англ. пер.: О.А. Ladyzhenskaya, The boundary value problems of mathematical physics, Appl. Math. Sci., 49, Springer-Verlag, New York, 1985.

24. О.А. Ладыженская, Н.Н. Уральцева, Линейнъе и көазилинейнъе уравнения эллиптического типа, Наука, М., 1964; англ. пер.: О. А. Ladyzhenskaya, N. N. Ural'tseva, Linear and quasilinear elliptic equations, Academic Press, New YorkLondon, 1968.

25. А. Н. Боголюбов, А. Л. Делицын, А. Г. Свешников, "О полноте корневых векторов радиоволновода", Докл. РАН, 369:4 (1999), 458-460; англ. пер.: А. N. Bogolyubov, A. L. Delitsyn, A. G. Sveshnikov, "On the completeness of root vectors of a radio waveguide", Dokl. Math., 60:3 (1999), 453-455.

26. А.Н. Боголюбов, А. Л. Делицын, А.Г Свешников, "Об условиях разрешимости задачи возбуждения волновода", Докл. РАН, 370:4 (2000), 453-456; англ. пер.: A. N. Bogolyubov, A. L. Delitsyn, A. G. Sveshnikov, "On conditions for the solvability of the problem of the excitation of a radio waveguide", Russian Acad. Sci. Dokl. Math., 61:1 (2000), 126-129.

27. V. Girault, P.-A. Raviart, Finite element methods for Navier-Stokes equations. Theory and algorithms, Springer Ser. Comput. Math., 5, Springer-Verlag, Berlin, 1986.

28. Ф. Рисс, Б. Секефальви-Надь, Лекции по функииональному анализу, Мир, М., 1979; пер. с фр.: F. Riesz, B. Sz.-Nagy, Leçons d'analyse fonctionnelle, GauthierVillars, Paris; Akadémiai Kiadó, Budapest, 1965.

29. Р. Курант, Д. Гильберт, Методы математической физики. Уравнения с частными производными, т. 2, Гостехиздат, М.-Л., 1945; пер. с нем.: R. Courant, D. Hilbert, Methoden der mathematischen Physik, 2, Julius Springer, Berlin, 1937.

30. М.В. Келдыш, "О собственных значениях и собственных функциях некоторых классов несамосопряженных уравнений”, ДАН СССР, 77:1 (1951), 11-14.

31. М.В. Келдыш, "О полноте собственных функций некоторых классов несамосопряженных линейных операторов”, УМН, 26:4 (1971), 15-41; англ. пер.: M. V. Keldysh, "On the completeness of the eigenfunctions of some classes of nonselfadjoint linear operators", Russian Math. Surveys, 26:4 (1971), 15-44.

32. А.Н. Боголюбов, А. Л. Делицын, М.Д. Малых, "О системе корневых векторов цилиндрического волновода", ЖВМ и $М \Phi, 41: 1$ (2001), 126-129; англ. пер.: A. N. Bogolyubov, A. L. Delitsyn, M. D. Malykh, "On the root vectors of a cylindrical waveguide", Comput. Math. Math. Phys., 41:1 (2001), 121-124.

33. Г.И. Веселов, С.Б. Раевский, Слоистые металло-диэлектрические волноводь, Радио и связь, М., 1988.

34. Г. И. Веселов, П. Е. Краснушкин, "О дисперсионных свойствах двухслойного экранированного круглого волновода и комплексных волнах в нем", ДАН СССР, 260:3 (1981), 576-579; англ. пер.: G. I. Veselov, P. E. Krasnushkin, "Dispersion properties of a two-layer screened round waveguide and complex waves in it", Soviet Phys. Dokl., 26:9 (1981), 835-837.

35. А.Л. Делицын, "Задача дифракции электромагнитного поля в цилиндрической области", Докл. РАН, 398:3 (2004), 310-313.

36. A.-S. Bonnet-Ben Dhia, P. Joly, "Mathematical analysis of guided water waves", SIAM J. Appl. Math., 53:6 (1993), 1507-1550. 
37. Р. З. Даутов, Е. М. Карчевский, “Об одной спектральной задаче теории диэлектрических волноводов", ЖВМ и МФ, 39:8 (1999), 1348-1355; англ. пер.: R. Z. Dautov, E.M. Karchevskiur, "A spectral problem in the theory of dielectric waveguides", Comput. Math. Math. Phys., 39:8 (1999), 1293-1299.

38. А.Л. Делицын, "О задаче рассеяния на неоднородности в волноводе", ЖЖВМ и МФ, 40:4 (2000), 606-610; англ. пер.: A. L. Delitsyn, "On the problem of scattering in a nonuniform waveguide", Comput. Math. Math. Phys., 40:4 (2000), 577-581.

39. D. S. Jones, "The eigenvalues of $\nabla^{2} u+\lambda u=0$ when the boundary conditions are on semi-infinite domains", Math. Proc. Cambridge Philos. Soc., 49 (1953), 668-684.

40. В. А. Бабешко, И. И. Ворович, И. Г. Образцов, "Явление высокочастотного резонанса в полуограниченных телах с неоднородностями", Механика твердого тела, 3 (1990), 74-84.

41. W. Bulla, F. Gesztesy, W. Renger, B. Simon, "Weakly coupled bound states in quantum waveguides", Proc. Amer. Math. Soc., 125:5 (1997), 1487-1495.

42. А.Л. Делицын, "О дискретном спектре оператора Лапласа в локально деформированном цилиндре”, Дифферени. уравн., 40:2 (2004), 198-207; англ. пер.: A. L. Delitsyn, "The discrete spectrum of the Laplace operator in a cylinder with locally perturbed boundary", Differ. Equ., 40:2 (2004), 207-217.

А. Л. Делицын (А. L. Delitsyn)

МГУ им. М. В. Ломоносова, физический ф-т

E-mail: delitsyn@mail.ru
Поступило в редакцию

01.12 .2004 